Issued by Sandia National Laboratories, operated for the United States Department of Energy by Sandia Corporation.

NOTICE: This report was prepared as an account of work sponsored by an agency of the United States Government. Neither the United States Government nor any agency thereof, nor any of their employees, nor any of their contractors, subcontractors, or their employees, makes any warranty, express or implied, or assumes any legal liability or responsibility for the accuracy, completeness, or usefulness of any information, apparatus, product, or process disclosed, or represents that its use would not infringe privately owned rights. Reference herein to any specific commercial product, process, or service by trade name, trademark, manufacturer, or otherwise, does not necessarily constitute or imply its endorsement, recommendation, or favoring by the United States Government, any agency thereof, or any of their contractors or subcontractors. The views and opinions expressed herein do not necessarily state or reflect those of the United States Government, any agency thereof, or any of their contractors.

Printed in the United States of America. This report has been reproduced directly from the best available copy.

Available to DOE and DOE contractors from Office of Scientific and Technical Information

P.O. Box 62

Oak Ridge, TN 37831

Prices available from (615) 576-8401, FTS 626-8401

Available to the public from

National Technical Information Service

U.S. Department of Commerce

5285 Port Royal Rd

Springfield, VA 22161

NTIS price codes

Printed copy: A04

Microfiche copy: A01

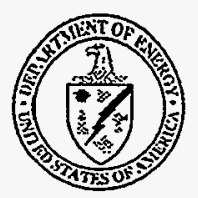




\section{DISCLAIMER}

Portions of this document may be illegible in electronic image products. Images are produced from the best available original document. 
SAND98-2630

Unlimited Release

Printed December 1998

\title{
Highly Dispersed Pseudo-Homogeneous and Heterogeneous Catalysts Synthesized via Inverse Micelle Solutions for the Liquefaction of Coal
}

\author{
Anthony Martino, Jeffrey S. Kawola, Allen G. Sault \\ Mark Hampden-Smith, Stacey A. Yamanaka \\ Catalysis and Chemical Technologies Department \\ Sandia National Laboratories \\ P.O. Box 5800 \\ Albuquerque, NM 87185-0710
}

\begin{abstract}
The mission of this project was to use inverse micelle solutions to synthesize nanometer sized metal particles and test the particles as catalysts in the liquefaction of coal and other related reactions. The initial focus of the project was the synthesis of iron based materials in pseudo-homogeneous form. The first three chapters discuss the synthesis, characterization, and catalyst testing in coal liquefaction and model coal liquefaction reactions of iron based pseudo-homogeneous materials. Later, we became interested in highly dispersed catalysts for coprocessing of coal and plastic waste. Bifunctional catalysts to hydrogenate the coal and depolymerize the plastic waste are ideal. We began studying, based on our previously devised synthesis strategies, the synthesis of heterogenous catalysts with a bifunctional nature. In chapter 4 , we discuss the fundamental principles in heterogenous catalysis synthesis with inverse micelle solutions. In chapter 5, we extend the synthesis of chapter 4 to practical systems and use the materials in catalyst testing. Finally in chapter 6 , we return to iron and coal liquefaction now studied with the heterogenous catalysts.
\end{abstract}




\section{Table of Contents}

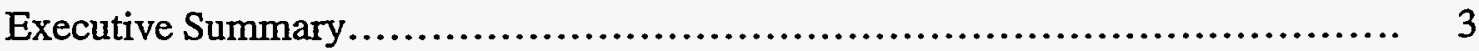

Chapter 1. Synthesis and Characterization of Coal Liquefaction

Catalysts in Inverse Micelles............................................

Chapter 2. Synthesis and Characterization of Coal Liquefaction Catalysts in Inverse Micelles II

Chapter 3. Preparation, Characterization, and Catalyst Testing of Highly Dispersed Rhodium Particles on Different Morphology Iron Oxide Supports 55

Chapter 4. Encapsulation of Gold Nanoclusters in Silica Materials via an Inverse Micelle / Sol-Gel Synthesis.

Chapter 5. Novel Sol-Gel Based Nanocluster Materials as Catalysts in the Dehydrogenation of Propane.

Chapter 6. Novel Sol-Gel Based Nanocluster Materials as Catalysts in the Liquefaction of Coal.

Appendix. Publications.................................................. 119 


\section{Executive Summary}

The mission of this project was to use inverse micelle solutions to synthesize nanometer sized metal particles and test the particles as catalysts in the liquefaction of coal and other related reactions. The initial focus of the project was the synthesis of iron based materials in pseudo-homogeneous form. The first three chapters discuss the synthesis, characterization, and catalyst testing in coal liquefaction and model coal liquefaction reactions of iron based pseudo-homogeneous materials. Later, we became interested in highly dispersed catalysts for coprocessing of coal and plastic waste. Bifunctional catalysts to hydrogenate the coal and depolymerize the plastic waste are ideal. We began studying, based on our previously devised synthesis strategies, the synthesis of heterogenous catalysts with a bifunctional nature. In chapter 4 , we discuss the fundamental principles in heterogenous catalysis synthesis with inverse micelle solutions. In chapter 5, we extend the synthesis of chapter 4 to practical systems and use the materials in catalyst testing. Finally in chapter 6 , we return to iron and coal liquefaction now studied with the heterogenous catalysts.

In chapter 1 , we discuss the synthesis of nanometer sized particles of $\mathrm{Fe}, \mathrm{Pd}$, and $\mathrm{FeS}_{2}$ (pyrite) in inverse micelle solutions and testing of the particles as catalysts in coal conversion processes. TEM shows that the smallest particles are less than $3 \mathrm{~nm}$ in diameter. Electron diffraction confirms a presence of $\alpha-\mathrm{Fe}$ (not crystalline oxides or borides), FCC-Pd, and the cubic pyrite structure of $\mathrm{FeS}_{2}$. Several chemical manipulation techniques have been devised to isolate the particles as powders. Methanol washing and forced precipitation causes uncontrolled growth of the particles and results in materials of relatively high surface area. The ultra-small clusters can be isolated in freeze dried surfactant powders, and these powders can be re-dispersed in a variety of organic solvents. Catalytic activity of the ultrasmall particles is determined in model compound hydrogenolysis, coal hydropyrolysis, and coal liquefaction. When surfactant is present, catalytic activity is hindered by two mechanisms. Surfactant chemically poisons the catalyst, and by-products of surfactant disintegration at reaction conditions scavenge hydrogen from hydrogen donating solvents. This latter mechanism is important in model compound hydrogenolysis and liquefaction as these processes depend strongly on hydrogen transfer. Moderate catalytic activity is observed in hydrogenolysis and liquefaction when the catalyst is used in a form free of 
surfactant. In coal hydropyrolysis, a process that does not depend on hydrogen donating solvents, significant catalytic activity is observed despite the presence of surfactant. We attribute the high activity to high surface areas of the ultra-small particles and increased contact between the coal and metal due to the wetting properties of the surfactant solutions.

Chapter 2 is a continuation of the work begun in chapter 1, but with more focus on the characterization of the Fe particles. We have synthesized nanometer sized, highly dispersed Fe based particles in inverse micelle systems. TEM shows that the average particle size is less than $3 \mathrm{~nm}$ in diameter. Mossbauer spectroscopy confirms that the products of the reduction reaction of iron salts with $\mathrm{LiBH}_{4}$ are $83 \% \mathrm{FeB}, 15 \% \mathrm{Fe}^{2+}{ }_{\mathrm{BO}}$, and $2 \% \mathrm{Fe}^{0}$. Methanol washing and forced precipitation causes uncontrolled growth of the particles and results in relatively high surface area powders. FeB is converted to $\alpha-\mathrm{Fe}$ then to an undetermined $\mathrm{Fe}^{2+}$ phase and then finally to $\mathrm{Fe}_{3} \mathrm{O}_{4}$ as the degree of oxidation by exposure to air increases. Exposure to methanol converts approximately $25 \%$ of $\mathrm{FeB}$ to $\mathrm{Fe} 0$. A shell and core picture of the clusters develops with oxidation as detected by XPS. Iron based metals are of interest as active, selective catalysts for a number of hydrogenation reactions including methanation and Fischer-Tropsch synthesis. We report again catalytic results of iron clusters in the hydrogenolysis of naphthyl bibenzyl methane (NBM), a model reaction for coal liquefaction.

In chapter 3, we study another methodology to synthesize highly dispersed metal particles on $\mathrm{Fe}$ based supports. We have prepared 2-4 $\mathrm{nm}$ sized $\mathrm{Rh}$ particles dispersed on a variety of supports by the photochemical reduction of $\left[\left(\mathrm{C}_{2} \mathrm{H}_{4}\right){ }_{2} \mathrm{Rh}(\mathrm{OEt})\right]_{2} \cdot \mathrm{H}_{2} \mathrm{O}$ in the presence of the supports. Rh particles were dispersed on support materials of different morphology and composition (rod-, spindle-, cube-shaped $\alpha-\mathrm{Fe}_{2} \mathrm{O}_{3}, \beta-\mathrm{FeOOH}$, and $\mathrm{SiO}_{2}$ ) indicating the procedure is general. The mild photochemical reduction does not effect the microstructure of the support. Quantitative deposition of the $\mathrm{Rh}$ on the support resulted as no unreacted $\left[\left(\mathrm{C}_{2} \mathrm{H}_{4}\right){ }_{2} \mathrm{Rh}(\mathrm{OEt})\right]_{2} \cdot \mathrm{H}_{2} \mathrm{O}$ or $\mathrm{Rh}$ particles exist in solution after reaction. Catalytic hydrogenation testing of the materials indicate that activity is a function of the composition of the support but not the morphology of the support at the length scales studied. Activity is also a function of particle size as effected by an increase in the metal loading of the material. Thermolysis of the $\beta-\mathrm{FeOOH}$ supported material shows that the dispersion of the metal and 
the morphology of the support remain constant, the crystalline $\beta$-FeOOH becomes amorphous, and a channel structure in the amorphous material develops. As a result, catalytic activity of the heat treated $\mathrm{Rh}$ on $\beta-\mathrm{FeOOH}$ decreases.

At this time in the project, we became interested in highly dispersed catalysts for coprocessing of coal and plastic waste. Bifunctional catalysts to hydrogenate the coal and depolymerize the plastic waste are ideal. We began studying, based on our previously devised synthesis strategies, the synthesis of heterogenous catalysts with a bifunctional nature. In chapter 4 , the discusssion is of nanometer sized gold particles encapsulated in the micropores of xerogels and aerogels. Gold was studied as a model system. The synthesis, characterization, and testing of practical materials are described in chapter 5. We have encapsulated nanometer sized Au particles in the micropores of xerogels and aerogels. The synthesis is a sequential reduction of a gold salt and sol-gel processing in an inverse micelle solution. The inverse micelle solution is used to solubilize the metal salt and provide a micro-reactor for the nucleation, growth, and stabilization of the nanometer sized clusters. Hydrolysis and condensation of an added siloxane precursor produces a wet gel embedding the particles. The presence of gel precursors de-stabilizes the inverse micelle structure resulting in larger particle sizes compared to typical inverse micelle synthesis techniques. Particle size control is complicated by the gel precursor effect on inverse micelle structure and the production of water and alcohol in the hydrolysis and condensation reactions. Finally, a unique gelation technique is outlined in these micro-heterogeneous solutions. Gelation occurs across the surfactant interface increasing the effective $\mathrm{H}_{2} \mathrm{O}: \mathrm{Si}$ ratio. Gelation occurs even at low $\mathrm{H}_{2} \mathrm{O}: \mathrm{Si}$ ratios, and condensation rates are high. Sol-gel parameters like the $\mathrm{H}_{2} \mathrm{O}: \mathrm{Si}$ ratio and the surfactant concentration have unique and sometimes non-intuitive effects on the hydrolysis and condensation rates and the resulting material properties.

Having now developed the theoretical foundations for the synthesis of heterogeneous catalysis based on an inverse micelle synthesis, in chapter 5 we turn to a practical catalyst system. We introduce two novel synthesis strategies to make nanoclusters on silica and alumina supports using inverse micelle technology and sol-gel processing. In the first methodology, sol-gel chemistry is performed in non-alcohol containing cluster, inverse micelle solutions. Hydrolysis and condensation reactions are inefficient, but nonetheless 
monoliths form around the clusters. In the second method, clusters formed in traditional inverse micelle solutions are allowed to diffuse into pre-existing monoliths formed using traditional sol-gel techniques (hydrolysis and condensation in ethanol). The two techniques give materials with similar cluster sizes, but different pore dimensions. At intermediate metal concentrations, particle sintering occurs even though metal size and pore dimensions are equal. At low metal concentrations, no appreciable sintering occurs even though the pores are much larger than the metal particles. We conclude that sintering is more a function of metal loading than the relative sizes of metal particles to pore diameters. Thus, even though sintering by particle to particle diffusion may be prevented by matching particle size with pore size, Ostwald ripening by atomic cleavage and migration from small to large particles dominates even in systems with equal particle and pore sizes. Our samples achieved relatively high catalytic activity and stability over time for propane dehydrogenation indicating that the synthesis methods described here result in catalyst performance more typical of $\mathrm{PtSn} / \mathrm{Al}_{2} \mathrm{O}_{3}$ or $\mathrm{PtSn} / \mathrm{SiO}_{2}$ catalysts prepared by impregnation. This behavior may indicate a high resistance to coke formation in our catalysts.

Finally, in chapter 6 we return to the study of coal liquefaction. We continue our systematic characterization and catalyst activity testing of highly dispersed heterogeneous nanostructured materials by applying the above developed technology to the synthesis of iron based supported xerogel materials. Several iron based samples were synthesized and tested as catalysts in coal liquefaction. One key element of the testing involved the control of the surface acidity of the support. Surface acidity provides the cracking sites for either coal liquefaction or coal-waste coprocessing. 


\title{
Chapter 1
}

\section{Synthesis and Characterization of Coal Liquefaction Catalysts in Inverse Micelles}

\begin{abstract}
We have synthesized nanometer sized particles of $\mathrm{Fe}, \mathrm{Pd}$, and $\mathrm{FeS}_{2}$ (pyrite) in inverse micelle solutions and tested the particles as catalysts in coal conversion processes. The synthesis procedure produces a variety of high surface area, highly dispersed, unsupported metal and metal compound catalysts. While Pd is used to test the viability of the particles formed by this synthesis technique, only disposable iron based particles are of economic interest. The particles are prepared by reduction or chemical reaction of salts solubilized in micro-heterogeneous surfactant and oil solutions. Chemical manipulation produces metal powders. Particle size and composition are determined by transmission electron microscopy, electron diffraction, and UV-visible spectrophotometry. Catalysts in solution and as powders are explored in three reactions: the hydrogenolysis of naphthyl bibenzyl methane, coal hydropyrolysis, and coal liquefaction. The model hydrogenolysis reaction provides information on the role of surfactant on catalytic activity. Results indicate good catalytic activity for coal hydropyrolysis and liquefaction.
\end{abstract}

\section{Introduction}

Surfactant molecules possess two distinct moieties: a hydrophilic head group and a hydrophobic tail group. Because of the dual nature of surfactant molecules, they selfassemble in various solvents 1 . In many two component systems of surfactant and oil, surfactants aggregate to form inverse micelles. Here, the hydrophilic head groups shield themselves by forming a polar core, and the hydrophobic tail groups are free to move about in the surrounding oleic phase. In three component microemulsion systems of water, oil, and surfactant, water is solubilized within the inverse micelle structure if the water concentration remains low. The solution takes on the structure of water droplets approximately 10-100 $\AA$ in 
diameter dispersed in the oleic solvent with surfactant forming the boundary between the two components.

In structured solutions such as inverse micellar and oil-rich microemulsion solutions, polar chemical reactants can be compartmentalized in the interior of the surfactant aggregates. Mixing two different solutions initiates a reaction sequence sustained by material exchange between inverse micelles. The surfactant interface provides a spatial constraint on the reaction volume, and reactions carried out in these micro-heterogeneous solutions produce colloidal sized particles (10-100 $)$ stabilized in solution against flocculation by surfactant $^{2,3}$. Metal alkoxide reactions ${ }^{4}$, the formation of polymer particles 5,6 , and the formation of metal ${ }^{7-12}$ and semiconductor ${ }^{13-21}$ clusters are examples of chemical processes that have been carried out in structured surfactant solutions.

The formation of ultra-small metal particles is of particular interest in the area of chemical catalysis. The clusters are high surface area, highly dispersed, unsupported materials. In addition, catalytic enhancement due to unique material properties (i.e. quantum size effects) is possible. Metal clusters prepared by a number of techniques have been studied as potential catalysts 22 . Reactant adsorption and the reactivity in various processes depends strongly on particle size. The effect is not just a matter of surface area, but of a fundamental change in the material's properties as the number of metal atoms per cluster decreases. $\mathrm{Pt}, \mathrm{Pd}, \mathrm{Rh}$, and Ir particles prepared via inverse micelle techniques ${ }^{23}$ have been studied in the hydrogenation and isomerization of but-1-ene 24,25 and the hydrogenolysis and isomerization of hexanes 26 . The catalysts were tested in-situ in surfactant, oil solutions and on supports. The activity of these catalysts increases when supported, and the selectivity of the colloidal sized particles is a function of the particle size.

We have studied the formation of iron based clusters and palladium clusters in inverse micelles and their use as catalysts in coal liquefaction reactions. Iron or palladium salts are solubilized within the polar interior of inverse micelles. The addition of an organic based reducing agent initiates a chemical reduction to produce $\mathrm{Fe}$ or $\mathrm{Pd}$ metal particles. The addition of a water based sulfiding agent initiates a chemical reaction to produce $\mathrm{FeS}_{2}$ (pyrite) semiconductor particles. The clusters are characterized with respect to size and elemental structure with transmission electron microscopy, electron diffraction, and UVvisible spectrophotometry. 
We have tested these iron based clusters for catalytic activity in a model reaction and in coal conversion reactions. The hydrogenolysis of naphthyl bibenzyl methane is used as a model for coal pyrolysis 27 . We have studied this reaction with our iron based clusters and with a commercial catalyst for comparison. The $\mathrm{Fe}, \mathrm{Pd}$, and $\mathrm{FeS}_{2}$ particles have also been tested in fixed bed coal hydropyrolysis and micro-batch coal liquefaction reactions. Once again, comparison is made with commercial catalysts.

\section{Experimental}

Octane, cyclohexane, and toluene were purchased from Aldrich at $99.9+\%$ purity. Surfactants used include didodecyldimethylammonium bromide (DDAB) from Kodak, butylethylene glycol n-dodecyl ether ( $\left.\mathrm{C}_{12} \mathrm{E}_{4}\right)$ from Nikkol, and POE (6) nonylphenol ( $\left.\mathrm{Ph9E} 6\right)$ from Chem Services Inc. Metal salts used include iron(II) chloride tetrahydrate, iron(III) chloride hexahydrate, iron(II) perchlorate hexahydrate, and palladium chloride from Aldrich, and iron(II) tetrafluoroborate hexahydrate from Alfa Chemicals. Lithium borohydride in tetrahydrofuran (2M) was purchased from Aldrich. Lithium sulfide was purchased from Aldrich and prepared in a $1 \mathrm{M}$ solution with de-ionized, distilled water.

The model hydrogenolysis compound, naphthyl bibenzyl methane (NBM), was synthesized in our laboratories using a previously developed recipe 28 . Coal hydropyrolysis was carried out on bituminous Herrin Burning Star No. 2, and coal liquefaction was carried out on DECS-17 Blind Canyon Coal obtained from the Penn State Coal Sample Bank. DECS-17 is a HVA bituminous coal with $0.36 \%$ iron, $0.02 \%$ pyritic sulfur, and $7.34 \%$ mineral matter (on a dry basis). Hydrogen donating solvents $1,2,3,6,7,8$ hexahydropyrene ( $\mathrm{H}_{6} \mathrm{Py}, 98 \%$ purity) and 9,10 dihydrophenanthrene (DHP, 94\% purity) were both purchased from Aldrich. Histological grade, stabilized tetrahydrofuran (THF) and reagent grade heptane from Fisher Scientific were used in product workups.

Synthesis of Metal Colloids ( $F e, P d$ ). Four different iron samples and two different palladium samples were prepared and studied (Table 1). The synthesis technique is general and specific samples differ only in the type and concentration of salts and surfactant added to the apolar solvents. First, the inverse micelle solutions are prepared by adding surfactant to the solvents (i.e. $\mathrm{DDAB}$ in toluene, $\mathrm{C}_{12} \mathrm{E}_{4}$ in octane). Then, the metal salts are introduced and the precursor salt solutions are mixed overnight on a stirring plate to assure complete 
solubilization. Transparent yellow Fe salt solutions are formed, and transparent clear Pd salt solutions are formed. $2 \mathrm{M} \mathrm{LiBH}_{4} / \mathrm{THF}$ solution is directly injected into the salt solutions under rapid stirring to initiate the reduction of iron or palladium. The reaction is run at a 5:1 molar ratio of $\left[\mathrm{BH}_{4}^{-}\right]:\left[\mathrm{Fe}^{3+}\right]$ or $\left[\mathrm{BH}_{4}^{-}\right]:\left[\mathrm{Pd}^{2+}\right]$. Fe solutions immediately turn clear and transparent or black. Pd solutions turn dark purple. The reactions are carried out under dry, oxygen-free conditions.

Two iron samples were further worked up to isolate the iron particles from solution. In the first sample (FeIII), $15 \mathrm{vol} . \%$ methanol was added to the particle solution. After several hours a black precipitate was formed at the bottom of the flask below a clear and transparent solution. The precipitate was separated from the solution by centrifugation and washed with methanol. The process was repeated two or three times, and the powder was finally dried. In the second sample (FeIV), the volatile liquid was striped off to form a surfactant paste that contained the iron clusters. Freeze-drying of the paste formed a particle embedded surfactant powder. In this sample, less surfactant was used to increase the metal concentration in the final powder. The powder is easily re-dispersed in organic solvents.

Synthesis of FeS2: Four different FeS2 samples were prepared and studied (Table 1). The synthesis is similar to the synthesis of metal colloids except $1 \mathrm{M} \mathrm{Li}_{2} \mathrm{~S}_{/} \mathrm{H}_{2} \mathrm{O}$ is injected into the salt precursor solutions rather than a reducing agent. The surfactant solutions must be chosen so that some degree of water can be solubilized. In FeS2IV, the precursor salt concentration was high enough that uncontrolled growth of the particles occurred upon reaction. The limited amount of surfactant was unable to stabilize particle growth, and the green product precipitated from solution. The particles were separated by centrifugation, washed, and dried to form a powder.

The samples used for UV-visible spectroscopy $\left(\mathrm{FeS}_{2} \mathrm{II}\right)$ were prepared with different amounts of water. The synthesis is slightly different here. The precursor salt is added to $\mathrm{H}_{2} \mathrm{O}$ first, and then the salt water is added to the surfactant, oil solution. The molarity of the salt in water and the amount of the salt water added to the inverse micelle solutions is adjusted to prepare samples of constant salt concentration and varying water amounts. The inverse micelle system ( $\mathrm{Ph}_{9} \mathrm{E}_{6} /$ cyclohexane) solubilizes a high degree of water. Two different synthesis routes were studied: (1) the reaction was run at a 5:1 molar ratio of $\left[\mathrm{S}_{2}{ }^{2-}\right.$ 
]:[Fe2+], (2) the reaction was run at a 1:1 molar ratio of $\left[\mathrm{S}_{2}{ }^{2-}\right]:\left[\mathrm{Fe}^{2+}\right]$. The solutions immediately turned dark green at low water concentrations and olive green at high water concentrations.

Characterization of the Colloids: The cluster solutions are studied by electron microscopy, electron diffraction, and UV-visible spectrophotometry. Particle size and structure are characterized with transmission electron microscopy (TEM) and electron diffraction. These tests are performed with a Joel 1200EX electron microscope. The colloidal solutions are applied directly on a holey carbon grid. The solution is wicked away by adsorbent towels under the grid and the particles are deposited on the grid. UV-visible spectrophotometry of the particle solutions is completed in-situ with a Hewlett-Packard $8452 \mathrm{~A}$ diode array spectrophotometer. BET surface area analysis of the methanol washed iron powder (FeIII) was carried out with a Quantachrome Autosorb-6 surface analysis apparatus.

Naphthyl Bibenzyl Methane (NBM) Hydrogenolysis: Hydrogenolysis testing of NBM has been developed as a model reaction for coal liquefaction 27 . We add $100 \mathrm{mg}$ of NBM, $400 \mathrm{mg}$ of the hydrogen donating solvent 9,10 dihydrophenanthrene $(9,10 \mathrm{DHP})$, and up to $15 \mathrm{wt}$. \% catalyst on an NBM basis to a $10 \mathrm{ml}$ flame sealed glass test tube. If used, $15 \mathrm{mg}$ of elemental sulfur is added. The test tube is sealed under ambient air pressure. The tube is placed in a $400^{\circ} \mathrm{C}$ sand bath for one hour. Previously, NBM hydrogenolysis has been used to study the selectivity of catalysts for five different cleavage reactions 27 . We do not quantify the selectivity of the catalysts used here. Conversion is calculated as the ratio of the molar sum of all products to the initial amount of NBM. The products are worked up in THF and analyzed by gas chromatography. Methanol washed and freeze dried Fe powders, and freeze dried $\mathrm{FeS}_{2}$ powders were tested as catalysts. These results were compared to the commercial catalyst Shell 324 (12.4 wt. \% Mo, 2.8 wt. \% Ni on $\mathrm{Al}_{2} \mathrm{O}_{3}$ ).

Coal Hydropyrolysis: Fixed-bed hydropyrolysis tests of bituminous Herrin Burning Star No. 2 were performed at $520^{\circ} \mathrm{C}$ and 150 bar of hydrogen 29 . Approximately $5 \mathrm{gm}$ of coal are mixed with $10 \mathrm{~g}$ sand in a reactor tube and heated at $5^{\circ} \mathrm{C} / \mathrm{sec}$. Char and ash are removed and weighed after each experiment. Conversion (100\% - \% char yield) is based on dry, mineral matter free coal. In-situ Fe and Pd colloids were tested as catalysts. The coal is wet with in-situ particle solutions, then dried, then the process is repeated until the desired 
amount of material is loaded on the coal. For comparison, a commercially available sulfided Mo catalyst is dispersed by wetting the coal with a solution of $\left(\mathrm{NH}_{4}\right)_{2} \mathrm{MoO}_{2} \mathrm{~S}_{2}$. This complex decomposes to $\mathrm{MoS}_{2}$ at $400^{\circ} \mathrm{C}$.

Coal Liquefaction: Batch liquefaction of Blind Canyon DECS-17 coal is performed in a stainless steel micro-reactor at a temperature of $400^{\circ} \mathrm{C}$, a cold pressure of $800 \mathrm{psi} \mathrm{H}_{2}$, and a reaction time of 30 minutes $30.2 .5 \mathrm{~g}$ of coal, $1 \mathrm{~g}$ of the hydrogen donating solvent hexahydropyrene, and up to $2.5 \mathrm{wt}$. \% of catalyst on a coal basis are added to the reactor. Reaction analysis consists of extracting products with THF and heptane (C7) in two separate stages, and the products are separated into categories depending on their solubility for the solvents. All soluble organic matter is extracted by THF (THF solubles), and the low molecular weight fraction of this material is extracted by $\mathrm{C} 7$ ( $\mathrm{C} 7$ solubles). Insoluble organic matter (IOM) is neither soluble in THF or C7. The difference between THF solubles and C7 solubles represents the amount of organic solubles not soluble in C7 (represented by THF solubles/C7 insolubles). The percentage of product refers to that percentage of the original amount of dry, mineral matter free coal added before reaction. Three particle samples were tested: freeze dried $\mathrm{Fe}$ and $\mathrm{FeS}_{2}$ samples as well as a surfactant free $\mathrm{FeS}_{2}$ powder (FeS $2 \mathrm{IV}$ ).

\section{Results}

Particle Characterization: Transmission electron micrographs of $\mathrm{Fe}$ and $\mathrm{FeS}_{2}$ particles show ultra-small particles of uniform size and shape highly dispersed on the grid (Figure 1). The number average diameter by TEM of FeI, PdI (not shown), and FeS2I particles is $1.5+/-0.2 \mathrm{~nm}, 1.8+/-0.2 \mathrm{~nm}$, and $3.1+/-0.1 \mathrm{~nm}$, respectively. Fe particle size as determined by TEM shows no discernible trend and is roughly constant in the ranges of $0.001 \mathrm{M}-0.01 \mathrm{M} \mathrm{FeCl} 3$ salt concentration and 1-10 wt. \% DDAB in toluene. Average particle size in this system is $2.4 \mathrm{~nm}$ in diameter. The size of FeS2 particles depends on the amount of water solubilized in the inverse micelle solutions and will be discussed later.

The methanol extracted Fe powder (FeIII) has been characterized by TEM and BET surface analysis. TEM pictures show agglomeration of the highly dispersed particles. Large agglomeration structures approximately $100 \mathrm{~nm}$ in size are observed. These structures 
consist of the ultra small Fe particles (approximately $2 \mathrm{~nm}$ in diameter) flocculated together. Multi-point BET analysis gives a surface area of $156 \mathrm{~m}^{2} / \mathrm{gm}$ for this sample.

Selected area electron diffraction patterns of FeII particles are consistent with the presence of $\alpha-\mathrm{Fe}$. The electron diffraction results are not consistent with the presence of any crystalline iron oxides or borides. Electron diffraction patterns of PdI particles are consistent with the presence of FCC-Pd. Electron diffraction patterns from $\mathrm{FeS}_{2} \mathrm{I}$ particles are consistent with the presence of cubic pyrite $\mathrm{FeS}_{2}$. Cubic pyrite $\mathrm{FeS}_{2}$ and cubic $\mathrm{FeS}$ have nearly equal theoretical scattering patterns, but the pattern we observe is distinguished from the pattern one gets from cubic FeS because of the presence of a scattering ring from the [220] plane in pyrite. This plane does not exist in cubic FeS. The electron diffraction pattern is not consistent with any other crystalline $\mathrm{Fe}$ and $\mathrm{S}$ compounds.

UV-visible spectra of the FeS2II particles in solution show four distinct absorption maxima (Figure 2a). When $\left[\mathrm{S}_{2}{ }^{2-}\right]:\left[\mathrm{Fe}^{2+}\right]$ is held in excess $(=5: 1)$, the absorption maxima shift to lower energies as the water content of the precursor inverse micelle microemulsion increases. When $\left[\mathrm{S}_{2}{ }^{2-]}:\left[\mathrm{Fe}^{2+}\right]=1\right.$, the maxima in the absorption spectra do not red shift with increasing water content, but decrease in magnitude and move toward the spectrum observed in the precursor salt solutions (Figure 2b). The absorption spectra also decay if the samples are held in the presence of oxygen over the course of a day (Figure 3).

Catalyst Testing: Hydrogenolysis of naphthyl bibenzyl methane (NBM) was tested with several inverse micelle synthesized iron based catalysts and compared with the commercial catalyst Shell 324. We tested the methanol extracted Fe powder (FeIII) with and without sulfur additives, and we tested freeze dried $\mathrm{Fe}$ and $\mathrm{FeS}_{2}$ particle embedded surfactant powders (FeIV and $\mathrm{FeS}_{2} \mathrm{IW}$ ). In each experiment we monitored the percentage recovery of . the reactant NBM and the percentage conversion to products 27 by gas chromatography. In each experiment we also monitored the percentage recovery of the hydrogen donating solvent 9, 10 DHP and the percentage converted to its dehydrogenated form phenanthrene. In an average of six runs, NBM was recovered at nearly $97+/-3 \%$ in thermal runs (no catalyst) and no product was obtained (Figures $4 \mathrm{a}$ and $\mathrm{b}$ ). Some NBM was converted to products in thermal runs with added elemental sulfur. Little 9, 10 DHP is dehydrogenated to phenanthrene in the thermal runs. Methanol extracted Fe powder as catalyst decreased the 
amount of NBM recovered to $87 \%$ and to $75 \%$ with added S. Product recovery correspondingly increased. In the Fe sample tests, hydrogens are given up to the hydrogenolysis products, and some 9,10 DHP is dehydrogenated to phenanthrene. FeIV and $\mathrm{FeS}_{2}$ III showed less catalytic activity according to the recovery of NBM. Product conversion could not be followed, however, because of the thermal cracking of the surfactant (bars not present in Figure 5a, D and E). Surfactant pyrolysis by-products disrupt GC analysis. Large amounts of the 9, 10 DHP was dehydrogenated to phenanthrene. Much higher product yields were obtained with Shell 324 (Figure 5). Higher conversions of 9,10 DHP to phenanthrene are consistent with these higher yields. In order to study the effect of surfactant on catalytic activity, we doped Shell 324 with increasing amounts of DDAB. As the Shell 324:DDAB mass ratio decreases, the trends in reactant recovery and product conversion move back to the thermal results indicating loss of catalytic activity. Initially the conversion of 9,10 DHP to phenanthrene moves toward the thermal results as well. At the lower Shell 324 : DDAB ratios, however, this trend reverses itself and nearly all of the 9,10 DHP is converted to phenanthrene.

Coal hydropyrolysis testing was done as a function of catalyst concentration for PdII catalysts. The coal was incipient wet as described previously to the desired active metal concentration. The activity of the catalyst increases with Pd concentration before flattening out at approximately $0.1 \%$ (Figure 6). The conversion at this point is roughly equal to that achieved with the commercial $\mathrm{MoS}_{2}$ catalyst. The weight percentage of the $\mathrm{MoS}_{2}$ catalyst is approximately $1 \%$ (not shown on Figure 6). Results for FeI particles are shown at one concentration and represent a lowering in overall coal conversion by approximately $10 \%$ compared to Pd results.

Freeze dried $\mathrm{Fe}$ and $\mathrm{FeS}_{2}$ particle embedded surfactant powders (FeIV and $\mathrm{FeS}_{2} \mathrm{III}$ ) and a surfactant free $\mathrm{FeS}_{2}$ powder ( $\mathrm{FeS}_{2} \mathbf{I V}$ ) were tested in micro-batch coal liquefaction reactions. Little to no improvement over the thermal runs is noticed in the freeze dried samples (Figure 7). The surfactant free $\mathrm{FeS}_{2}$ results show substantial improvement in the conversion to $\mathrm{C} 7$ solubles. Overall conversion to THF solubles decreases.

\section{Discussion}


Catalyst Synthesis and Characterization: Introduction of $\mathrm{LiBH}_{4}$ to the inverse micelle solutions containing iron or palladium salts causes immediate reduction to $\mathrm{Fe}$ or $\mathrm{Pd}$, respectively. The particles are formed by the following chemical reactions: $\left[\mathrm{Fe}^{3+}\right]+3\left[\mathrm{H}^{-}\right]-$ $\rightarrow \mathrm{Fe}$ (particles) $+3 / 2 \mathrm{H}_{2}$ and $\left[\mathrm{Pd}^{2+}\right]+2\left[\mathrm{H}^{-}\right] \rightarrow \mathrm{Pd}$ (particles) $+\mathrm{H}_{2}$. Introduction of $\mathrm{Li} 2 \mathrm{~S}$ to the Fe salt solubilized inverse micelle solutions causes immediate sulfiding of the Fe. The particles are formed by the following reaction: $\left[\mathrm{Fe}^{2+}\right]+\left[\mathrm{S}_{2}{ }^{2-}\right] \rightarrow \mathrm{FeS}_{2}$ (particles). Characterization of the particles by electron diffraction give strong evidence that the chemistry described here produces $\alpha-\mathrm{Fe}, \mathrm{FCC}-\mathrm{Pd}$, and $\mathrm{FeS}_{2}$ (pyrite). $\mathrm{LiBH}_{4}$ or $\mathrm{Li}_{2} \mathrm{~S}$ enters the surfactant, oil solution and is solubilized within the inverse micelle structure. The reaction is initiated and is sustained through material exchange between inverse micelles. After the particle growth has equilibrated, the surfactant stabilizes the particles and prevents flocculation and precipitation.

The final particle size of nanometer sized clusters formed in inverse micelles depends on a complicated nucleation and growth process 31 . Inverse micelles effect this process primarily in two ways: (1) diffusion of the reacting nucleation sites and ions is governed by exchange rates between micelles and (2) the critical nucleation size depends inherently on the size of the inverse micelles. Size dependence of $\mathrm{Au}, \mathrm{CdS}$, and CdSe clusters produced in inverse micelles has been discussed previously32,13-21. The Fe particles synthesized in $\mathrm{DDAB} /$ toluene mixtures showed no size dependence by TEM on the salt to surfactant concentration ratio within the range studied.

Size dependence of $\mathrm{FeS}_{2}$ particles on water concentration in the precursor inverse micelle solutions is inferred from UV-visible spectrophotometry. Particle growth is observed by UV-visible spectrophotometry, because semiconductor band gap excitations in the UVvisible region decrease with increasing particle size when quantum size effects are important $33-35$. We have observed that the absorption maxima red shift in $\mathrm{FeS}_{2}$ with increasing water concentration (Figure 2a). We attribute the red shift in the UV-visible spectra with increasing water concentration to the formation of larger particles primarily due to the increase in inverse micelle size. This conclusion assumes the absorption maxima in $\mathrm{FeS}_{2}$ (pyrite), a semiconductor, is due to a complicated photogenerated band gap excitation in the material. If so, we have illustrated that the $\mathrm{FeS}_{2}$ particles are small enough to exhibit 
quantum confinement effects, and that we can increase $\mathrm{FeS}_{2}$ particle size by increasing the water concentration in inverse micelle microemulsions.

The chemical sensitivity of $\mathrm{FeS}_{2}$ particles in solution is illustrated by the dependence of the absorption maxima on reactant concentration and exposure to air. The absorption maxima in $\mathrm{FeS}_{2}$ do not shift with increasing water concentration when the $\left[\mathrm{S}_{2}{ }^{2-}\right]\left[\mathrm{Fe}^{2+}\right]$ molar ratio is not in excess (Figure 2b). The maxima decrease, and at the highest water concentration the spectra resembles the spectra of the iron salt solution. We conclude that the reaction governing the production of $\mathrm{FeS}_{2}$ colloids is reversible, and that water in the microemulsion mixtures favor the decomposition of the colloids into the ions. It is necessary to use excess reactants in order to push the chemical reaction to the right and favor the formation of the particles. The high surface reactivity of CdSe particles has been demonstrated previously 31 . The stability of the $\mathrm{FeS}_{2}$ particles further demonstrates surface reactivity. Oxidation of $\mathrm{FeS}_{2}$ is observed by the disappearance of the absorption maxima when the sample is left in the presence of air. Oxidation of Fe clusters is visible to the naked eye as the solutions turn yellow if exposed to air.

Catalyst Testing: Hydrogenolysis of NBM indicates catalytic activity with FeIII. A more than linear increase in activity with added $\mathrm{S}$ indicates that there is a synergistic effect between the $\mathrm{Fe}$ and $\mathrm{S}$.

Catalyst activity of Shell 324 far exceeds the activity of the iron based clusters. Doping Shell 324 with surfactant illustrates the effect of surfactant on catalyst activity. When Shell 324 is doped with surfactant, catalyst activity decreases (Figure 5). The behavior of the hydrogen donating solvent indicates two mechanisms are at work. At low surfactant concentrations, a decrease in product conversion and a decrease in the amount of 9, $10 \mathrm{DHP}$ dehydrogenated to phenanthrene indicates less activity due to a chemical or steric poisoning of the catalyst. At high surfactant concentrations, we propose that a decrease in NBM conversion and an increase in the amount of 9,10 DHP dehydrogenated to phenanthrene indicates that the thermal pyrolysis products of the surfactant (observed by GC) scavenge the hydrogens from the donor solvent. Decreased activity with high dehydrogenation conversion of 9,10 DHP to phenanthrene of FeIV and FeS2 III is consistent with the presence of surfactant and the above stated mechanism. 
Coal hydropyrolysis tests indicate that catalytic activity of ultra-small Pd particles rivals the activity of the $\mathrm{MoS}_{2}$ catalyst. The adverse effect of surfactant is less important in hydropyrolysis. Hydropyrolysis is independent of hydrogen transfer from a hydrogen donating solvent, thus eliminating a mechanism by which surfactant hinders the reaction. Any negative effect of surfactant may also be offset by increased contact of metal and coal due to wetting properties of the surfactant solution. Fel particles show relatively high conversions, but less than $\mathrm{Pd}$ and the commercial catalyst. The activity is significant considering the concentration of $\mathrm{Fe}$ used is more than an order of magnitude lower than the concentration of the $\mathrm{MoS}_{2}$ catalyst.

Iron sulfide has been studied extensively as a coal liquefaction catalyst. It is believed that pyrrhotite $\left(\mathrm{Fe}_{1-\mathrm{x}} \mathrm{S}\right)$ is the active form of the iron catalyst ${ }^{36}$, and that pyrite is reduced to pyrrhotite under liquefaction conditions 37 . We have attempted to use our $\mathrm{Fe}$ and $\mathrm{FeS}_{2}$ (pyrite) particles as coal liquefaction catalysts. FeIV and FeS $_{2} \mathrm{III}$ powders show no improvement over thermal reactions. Liquefaction is highly dependent on a hydrogen donating solvent and surfactant pyrolysis by-products may scavenge hydrogen. Surfactant free $\mathrm{FeS}_{2} \mathrm{IV}$ powder shows a decrease in the overall conversion to organic products, but an actual increase in the amount of low molecular weight organic products. High IOM percentages may be due to high selectivity for cracking polar functionalities within DECS17.

\section{Conclusions}

We have synthesized ultra-small, highly dispersed $\mathrm{Fe}, \mathrm{Pd}$, and $\mathrm{FeS}_{2}$ (pyrite) particles in a variety of inverse micelle systems. TEM shows that the smallest particles are less than 3 $\mathrm{nm}$ in diameter. Electron diffraction confirms a presence of $\alpha-\mathrm{Fe}$ (not crystalline oxides or borides), FCC-Pd, and the cubic pyrite structure of $\mathrm{FeS}_{2}$. Several chemical manipulation techniques have been devised to isolate the particles as powders. Methanol washing and forced precipitation causes uncontrolled growth of the particles and results in materials of relatively high surface area. The ultra-small clusters can be isolated in freeze dried surfactant powders, and these powders can be re-dispersed in a variety of organic solvents. Catalytic activity of the ultra-small particles is determined in model compound hydrogenolysis, coal 
hydropyrolysis, and coal liquefaction. When surfactant is present, catalytic activity is hindered by two mechanisms. Surfactant chemically poisons the catalyst, and by-products of surfactant disintegration at reaction conditions scavenge hydrogen from hydrogen donating solvents. This latter mechanism is important in model compound hydrogenolysis and liquefaction as these processes depend strongly on hydrogen transfer. Moderate catalytic activity is observed in hydrogenolysis and liquefaction when the catalyst is used in a form free of surfactant. In coal hydropyrolysis, a process that does not depend on hydrogen donating solvents, significant catalytic activity is observed in spite of the presence of surfactant. We attribute the high activity to high surface areas of the ultra-small particles and increased contact between the coal and metal due to the wetting properties of the surfactant solutions. 


\section{References}

(1) Langevin, D. Accounts of Chemical Research 1988, 21, 255-260.

(2) Fendler, J.H. Chem. Rev. 1987, 87, 877-899.

(3) Henglein, A. Chem. Rev. 1989, 89, 1861-1873.

(4) Jean, J.H.; Ring, T.A. Colloids and Surfaces 1988, 29, 273-291.

(5) Paleos, C.M. Chemical Society Reviews, 14, 45-67.

(6) Perez-Luna, V.H.; Puig, J.E.; Castano, V.M.; Rodriguez, B.E.; Murthy, A.K.; Kaler,

E.W. Langmuir 1990, 6, 1040-1044.

(7) Barnickel, P.; Wokaun, A. Molecular Physics 1990, 69, 1-9.

(8) Gobe, M; Kon-No, K.; Kandori, K.; Kitahara, A. Journal of Colloid and Interface

Science 1983, 93, 293-295.

(9) Kurihara, K.; Kizling, J.; Stenius, P.; Fendler, J.H. J. Am. Chem. Soc. 1983, 105, 25742579.

(10) Nagy, J.B.; Gourgue, A. Derouane, F.G. Preparation of Catalysts III; eds. Grange, P.; Jacobs, P.A.; Elsevier Science Publishers, 1983; pp 193-202.

(11) Nagy, J.B. Colloids and Surfaces 1989, 35, 210-220.

(12) Torigoe, K.; Esumi, K. Langmuir 1992, 8, 59-63.

(13) Lianos, P.; Thomas, J.K. Journal of Colloid and Interface Science 1987, 117, 505-512.

(14) Lianos, P.; Thomas, J.K. Chemical Physics Letters 1986, 125, $299-302$.

(15) Modes, S.; Lianos, P. J. Phys. Chem. 1989, 93, 5854-5859.

(16) Petit, C.; Pileni, M.P. J. Phys. Chem. 1988, 92, 2282-2286.

(17) Petit, C.; Lixon, P.; Pileni, M.P. J. Phys. Chem. 1990, 94, 1598-1603.

(18) Motte, L.; Petit, C.; Boulanger, L.; Lixon, P.; Pileni, M.P. Langmuir 1992, 8, 10491053.

(19) Pileni, M.P.; Motte, L.; Petit, C. Chem. Mater. 1992, 4, 338-345.

(20) Robinson, B.H.; Towey, T.F.; Zourab, S.; Visser, A.J.W.G.; van Hoek, A. Colloids and Surfaces 1991, 61, 175-188.

(21) Towey, T.F.; Khan-Lodhi, A.; Robinson, B.H. J. Chem. Soc. Faraday Trans. 1990, 86, 3757-3762.

(22) Moskovits, M. Annu. Rev. Phys. Chem. 1991, 42, 465-499.

(23) Boutonnet, M.; Kizling, J.; Stenius, P.; Maire, G. Colloids and Surfaces 1982, 5, 209. 225.

(24) Boutonnet, M.; Kizling, J.; Touroude, R.; Maire, G.; Stenius, P. Applied Catalysis 1986, 20, 163-177.

(25) Boutonnet, M.; Kizling, J.; Mintsa-Eya, V.; Choplin, A.; Touroude, R.; Maire, G.; Stenius, P. Journal of Catalysis 1987, 103, 95-104.

(26) Boutonnet, M.; Kizling, J.; Touroude, R.; Maire, G.; Stenius, P. Catalysis Letters 1991, 9, 347-354.

(27) Faracasiu, M.; Smith, C. ACS Preprints Fuel Chemistry Division 1990, 35, 404.

(28) Faracasiu, private communication.

(29) Snape, C.E.; Bolton, C.; Dosch, R.G.; Stephens, H.P. Energy and Fuels 1989, 3, 421 425.

(30) Stohl, F.V.; Diegert, K.V. Energy and Fuels 1994, 8, 117-123.

(31) Steigerwald, M.L.; Brus, L.E. Annu. Rev. Mater. Sci. 1989, 19, 471-493. 
(32) Wilcoxon, J.P., Williamson, R.L., Baughman, R. J. Chem. Phys. 1993, 98, 9933-50. (33) Brus, L. J. Phys. Chem. 1986, 90, 2555-2560.

(34) Rossetti, R.; Ellison, J.L.; Gibson, J.M.; Brus, L.E. J. Chem. Phys. 1984, 80, 44644469.

(35) Wang, Y.; Herron, N. J. Phys. Chem. 1991, 95, 525-532.

(36) Davidson, R.M. IEA Coal Research 1983, 14, 376.

(37) Kotanigawa, T.; Yokoyama, S.; Yamamoto, M.; Maekawa, Y. Fuel 1987, 66, 1452. 
Table 1. Listing of the samples prepared and used in this study.

\begin{tabular}{|c|c|c|}
\hline Name & Description & Uses \\
\hline FeI & $0.01 \mathrm{M} \mathrm{FeCl}_{3}, 10$ wt. \% DDAB/toluene & TEM, Hydropyrolysis \\
\hline FeII & $0.01 \mathrm{Fe}\left(\mathrm{BF}_{4}\right)_{2}, 10$ wt. $\% \mathrm{C}_{12} \mathrm{E}_{4} /$ octane & Electron Diffraction \\
\hline FeIII & FeI, powder from methanol washing & TEM, Hydrogenolysis, BET \\
\hline FeIV & $\begin{array}{l}0.01 \mathrm{M} \mathrm{FeCl}_{3}, 5 \mathrm{wt} . \% \mathrm{DDAB} / \text { toluene, } \\
\text { freeze dried powder }\end{array}$ & Hydrogenolysis, Liquefaction \\
\hline PdI & $0.005 \mathrm{M} \mathrm{PdCl}_{2} 10 \mathrm{wt} . \% \mathrm{DDAB} /$ toluene & TEM, Electron Diffraction \\
\hline PdII & $0.01 \mathrm{M} \mathrm{PdCl}_{2} 10 \mathrm{wt} . \% \mathrm{DDAB} /$ toluene & Hydropyrolysis \\
\hline $\mathrm{FeS}_{2} \mathrm{I}$ & $0.001 \mathrm{M} \mathrm{Fe}\left(\mathrm{ClO}_{4}\right)_{2}, 10$ wt. $\% \mathrm{C}_{12} \mathrm{E}_{4} /$ octane & TEM, Electron Diffraction \\
\hline $\mathrm{FeS}_{2} \mathrm{II}$ & $\begin{array}{l}0.001 \mathrm{M} \mathrm{FeCl}_{2}, 10 \text { wt. \% } \\
\mathrm{Ph}_{9} \mathrm{E}_{6} / \text { cyclohexane, w/ } \mathrm{H}_{2} \mathrm{O}\end{array}$ & $\begin{array}{l}\text { UV-Visible } \\
\text { Spectrophotometry }\end{array}$ \\
\hline $\mathrm{FeS}_{2} \mathrm{III}$ & $\begin{array}{l}0.01 \mathrm{M} \mathrm{Fe}\left(\mathrm{ClO}_{4}\right)_{2}, 10 \mathrm{wt} . \% \mathrm{C}_{12} \mathrm{E}_{4} / \text { octane, } \\
\text { freeze dried powder }\end{array}$ & Hydrogenolysis, Liquefaction \\
\hline $\mathrm{FeS}_{2} \mathrm{IV}$ & $\begin{array}{l}0.05 \mathrm{M} \mathrm{Fe}\left(\mathrm{ClO}_{4}\right)_{2}, 10 \mathrm{wt} . \% \mathrm{C}_{12} \mathrm{E}_{4} / \text { octane, } \\
\text { uncontrolled precipitate powder }\end{array}$ & Liquefaction \\
\hline
\end{tabular}




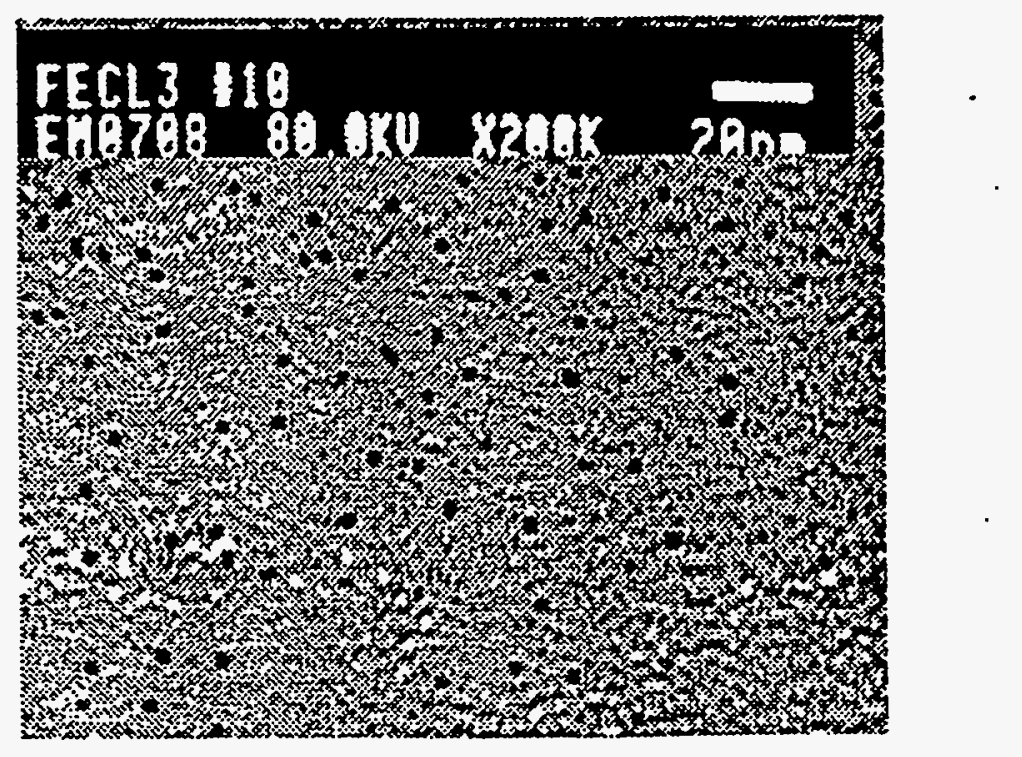

Figure 1a.

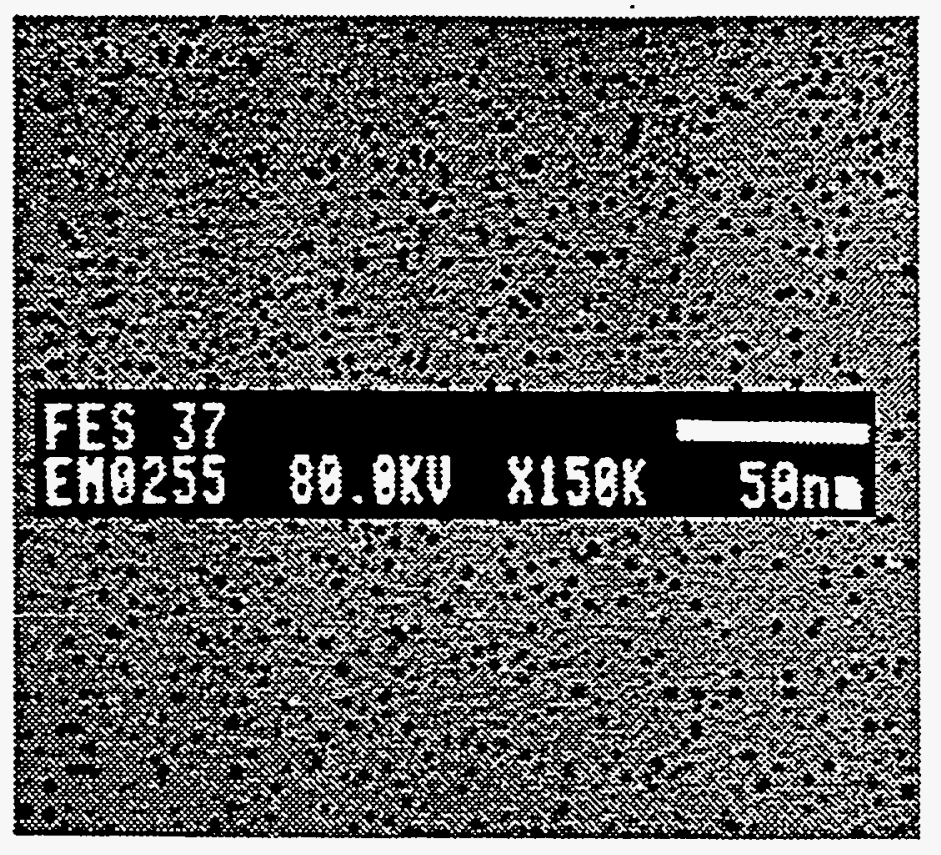

Figure 1b.

Figure 1. (a) TEM of FeI particles. The number average diameter of the particles shown is $1.5+/-0.2 \mathrm{~nm}$. (b) TEM of $\mathrm{FeS}_{2} \mathbf{I}$. The number average diameter of the particles shown is $3.1+/-0.2 \mathrm{~nm}$. 


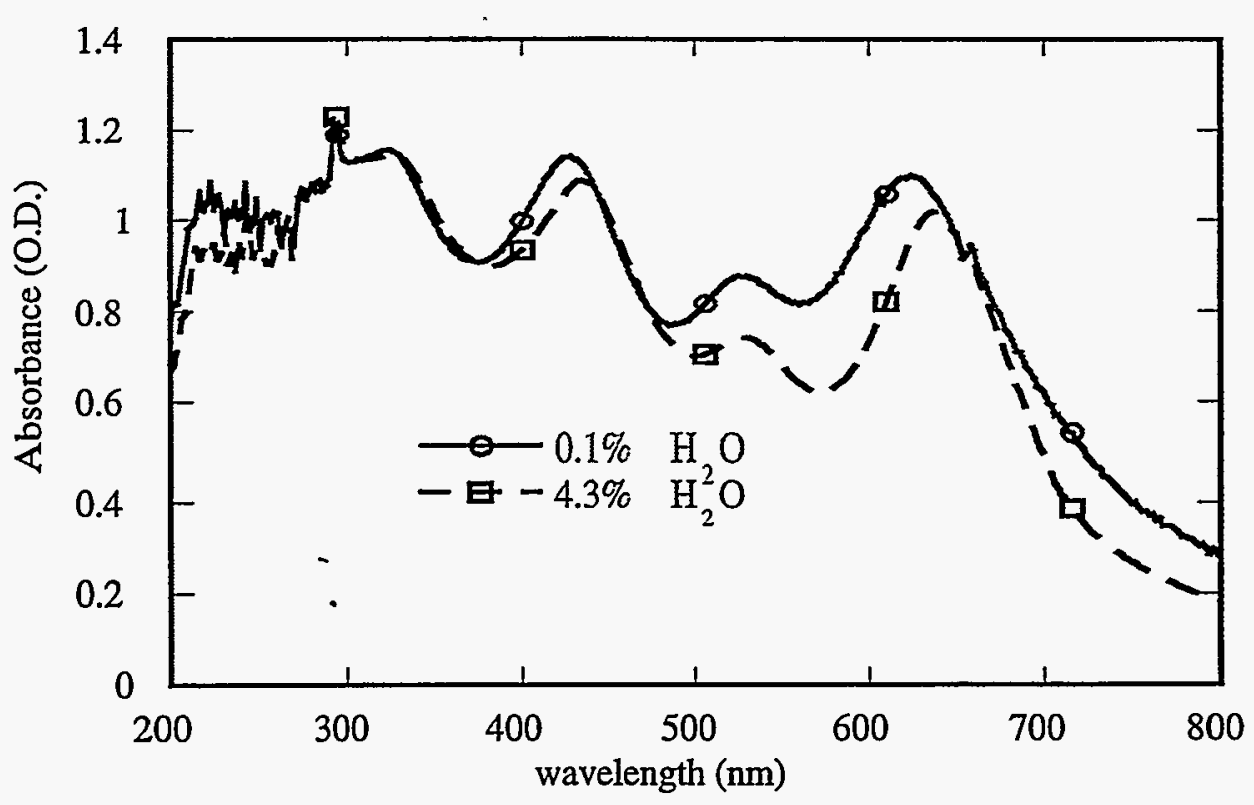

a)

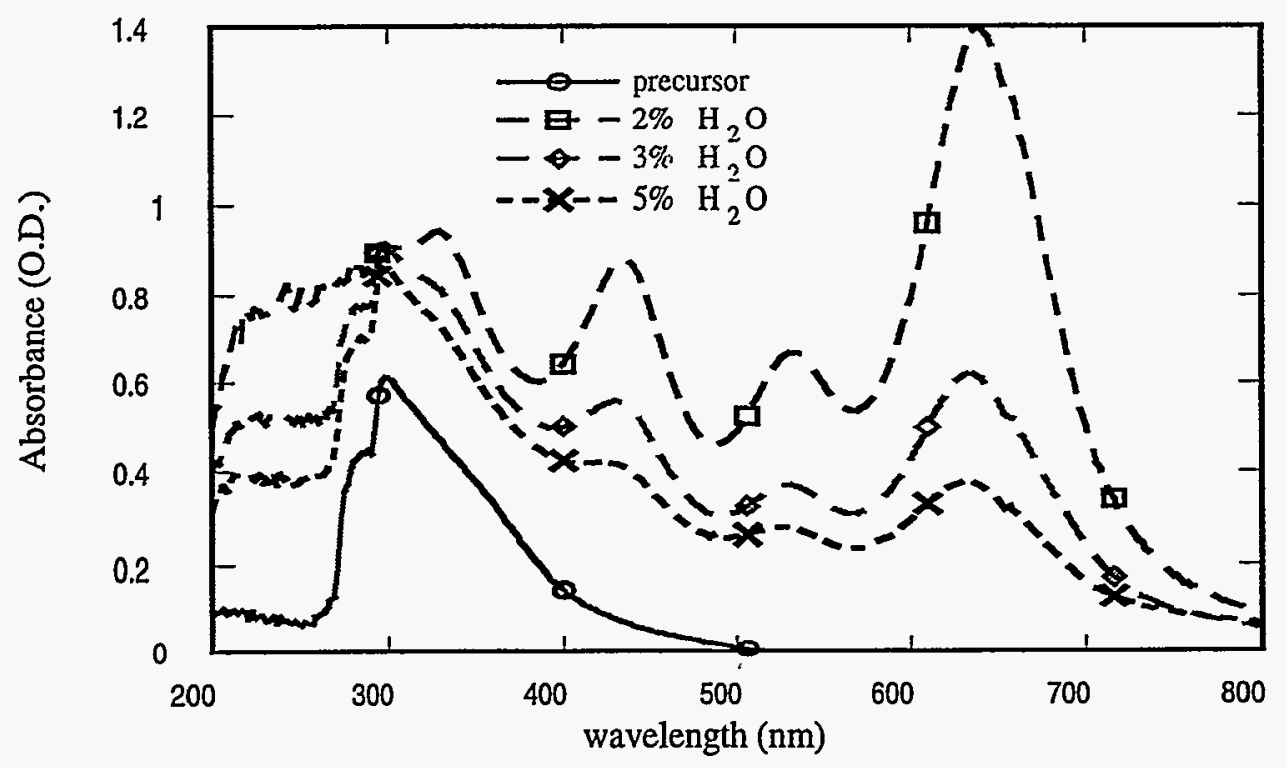

b)

Figure 2. UV-visible spectra of $\mathrm{FeS}_{2} \mathrm{II}$ particles in solution. (a) $\left[\mathrm{S}_{2}{ }^{2-}\right]:\left[\mathrm{Fe}^{2+}\right]=5: 1$. The electronic spectra of the semiconductor shifts to lower energies with increasing water concentration in the inverse micelle solutions. (b) $\left[\mathrm{S}_{2}{ }^{2-}\right]:\left[\mathrm{Fe}^{2+}\right]=1: 1$. With increasing water concentration, the spectra contains elements of $\mathrm{FeS}_{2}$ colloids and the precursor salt. 


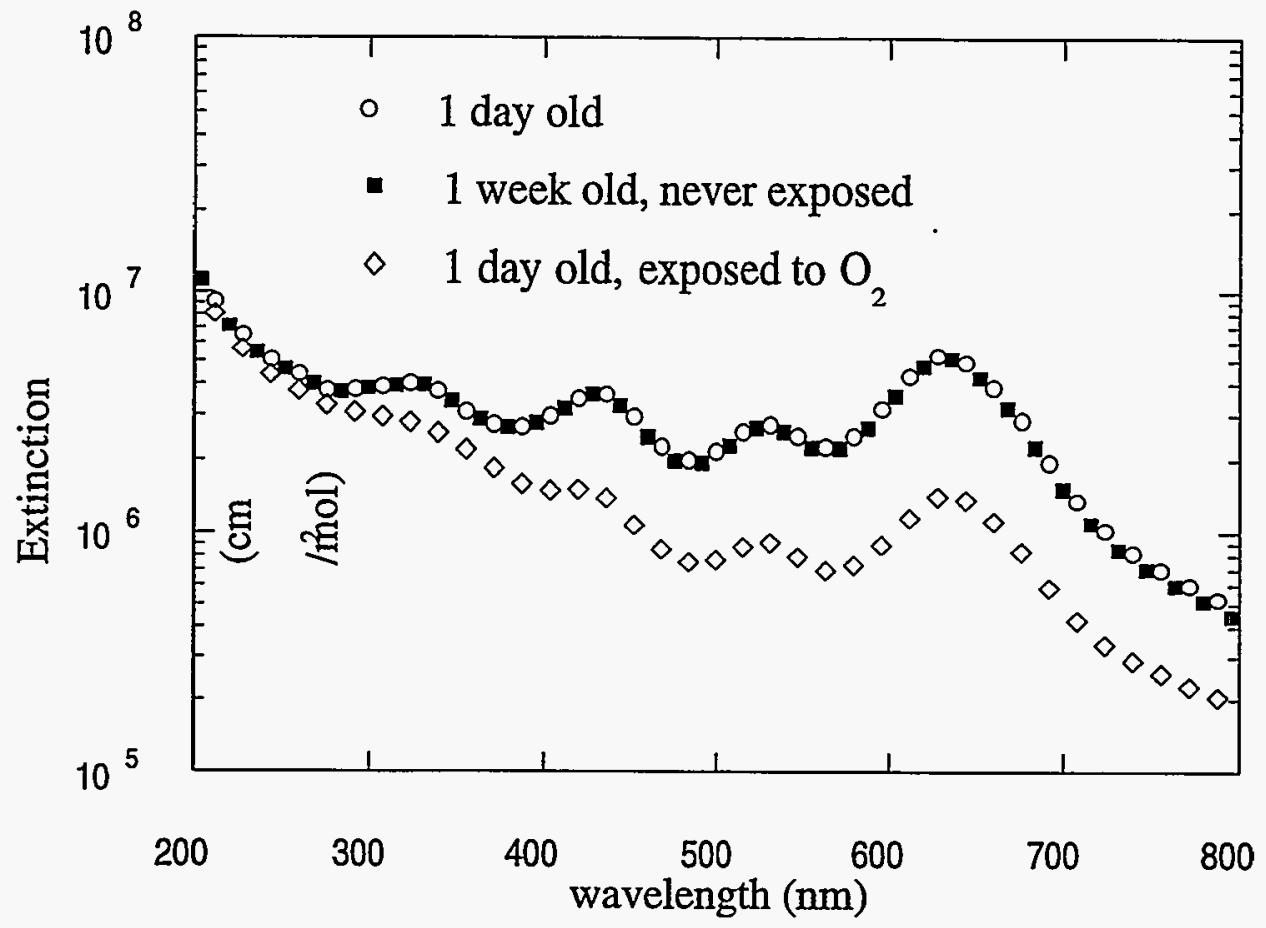

Figure 3. UV-visible spectra of $\mathrm{FeS}_{2} \mathrm{II}$ particles in solution. The colloids are stable in the absence of air, but degrade when exposed to $\mathrm{O}_{2}$. 


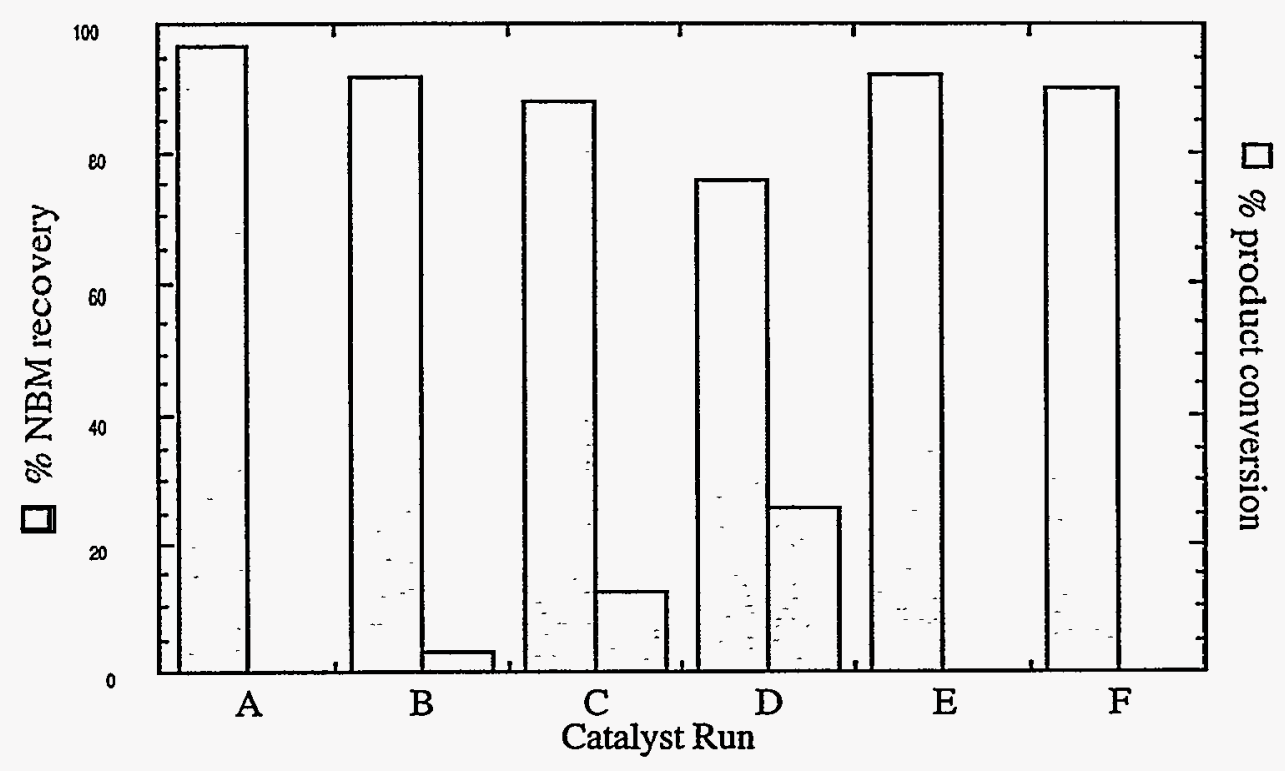

a)

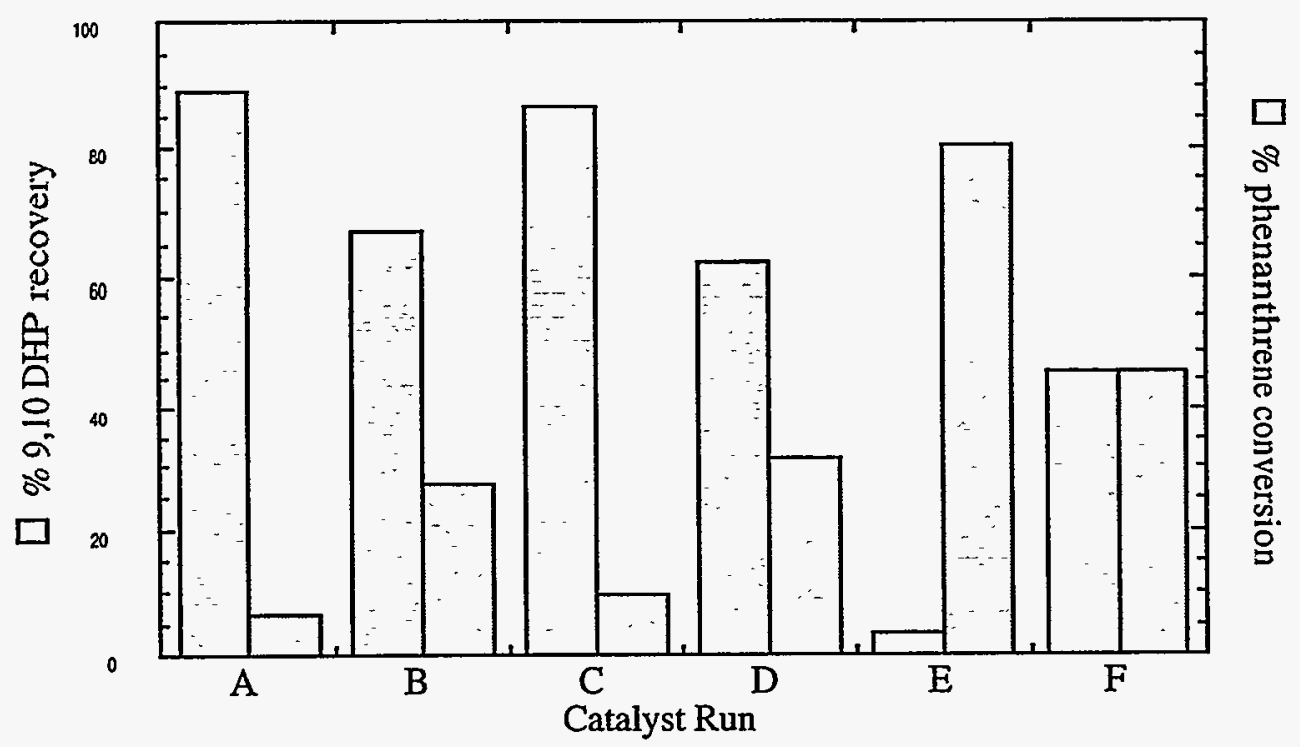

b)

Figure 4. NBM hydrogenolysis results for Fe clusters. (a) The percentage of reactant recovered and percentage converted to product. (b) The percentage of the hydrogen donating solvent $9,10 \mathrm{DHP}$ recovered and the percentage dehydrogenated to phenanthrene. $\mathrm{A}=$ thermal average; $\mathrm{B}=$ thermal with elemental $\mathrm{S}$ average; $\mathrm{C}=$ FeIII ( $\sim 15 \%$ active metal basis); $\mathrm{D}=\mathrm{FeIII}$ with elemental $\mathrm{S}(\sim 15 \%$ active metal basis); $\mathrm{E}=\mathrm{FeIV}(\sim 1.8 \%$ active metal basis); $\mathrm{F}=\mathrm{FeS} 2 \mathrm{III}(\sim 1 \%$ active metal basis). 


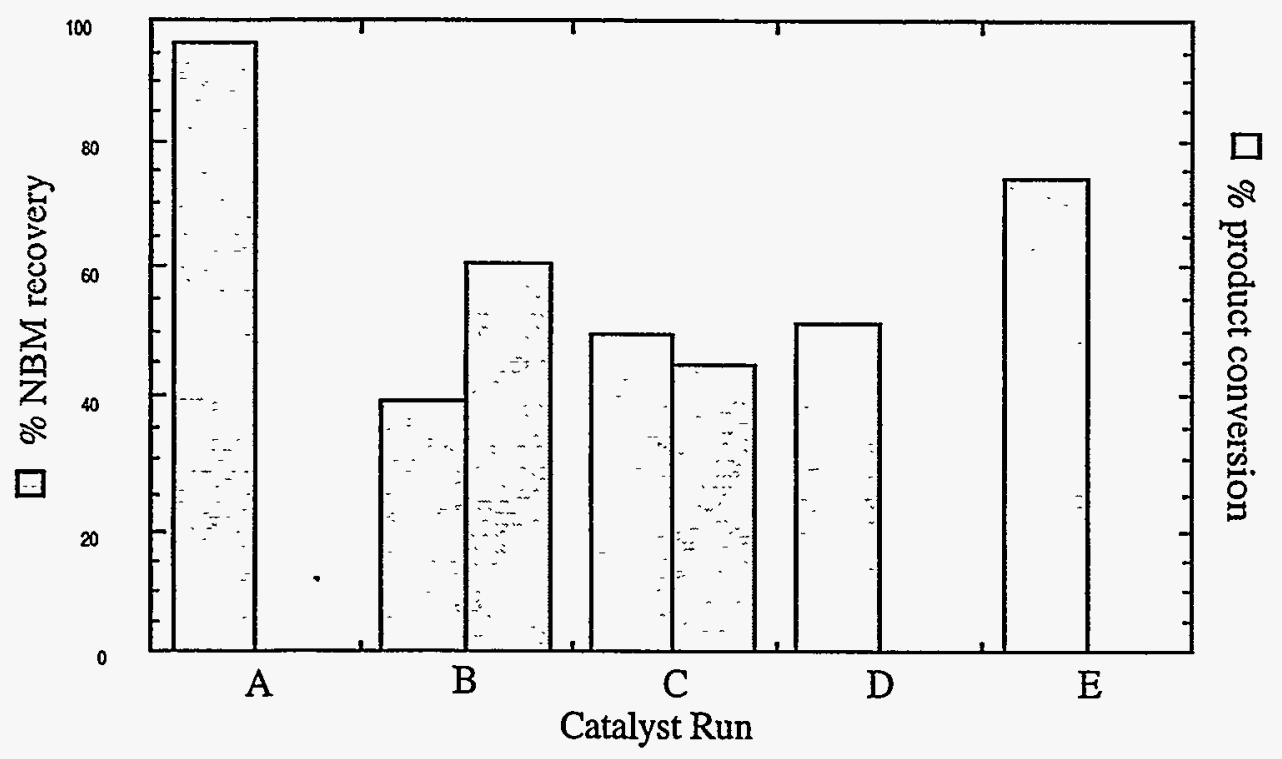

a)

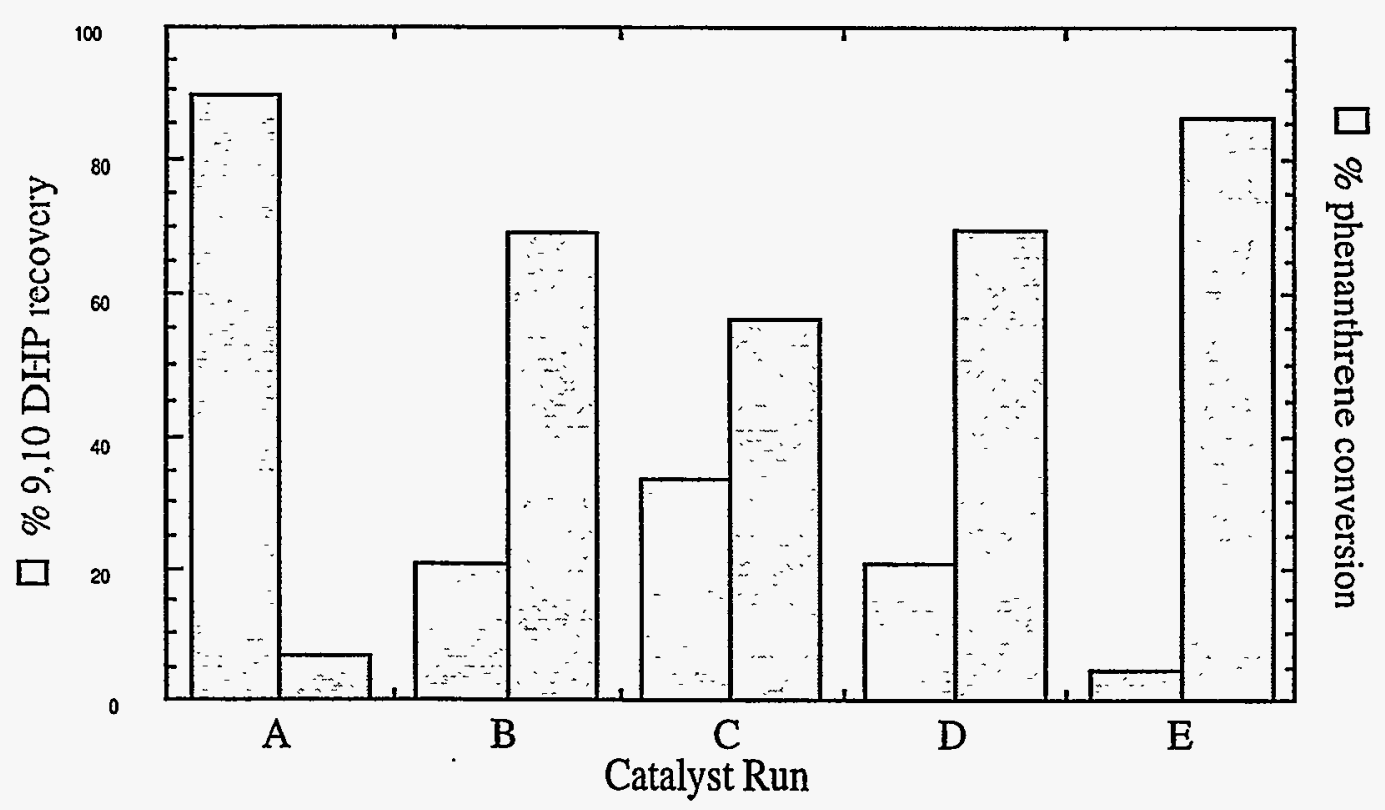

b)

Figure 5. NBM hydrogenolysis results for Shell 324 as a function of DDAB surfactant doping. (a) The percentage of reactant recovered and percentage converted to product. (b) The percentage of the hydrogen donating solvent $9,10 \mathrm{DHP}$ recovered and the percentage reduced to phenanthrene. $\mathrm{A}=$ thermal average; $\mathrm{B}=$ Shell 324; $\mathrm{C}=$ Shell 324:DDAB $=1: 4 ; \mathrm{D}=$ Shell 324:DDAB $=1: 20 ; \mathrm{E}=$ Shell 324:DDAB $=1: 50$. Active metal basis $\sim 15 \%$ for all catalyst runs. 


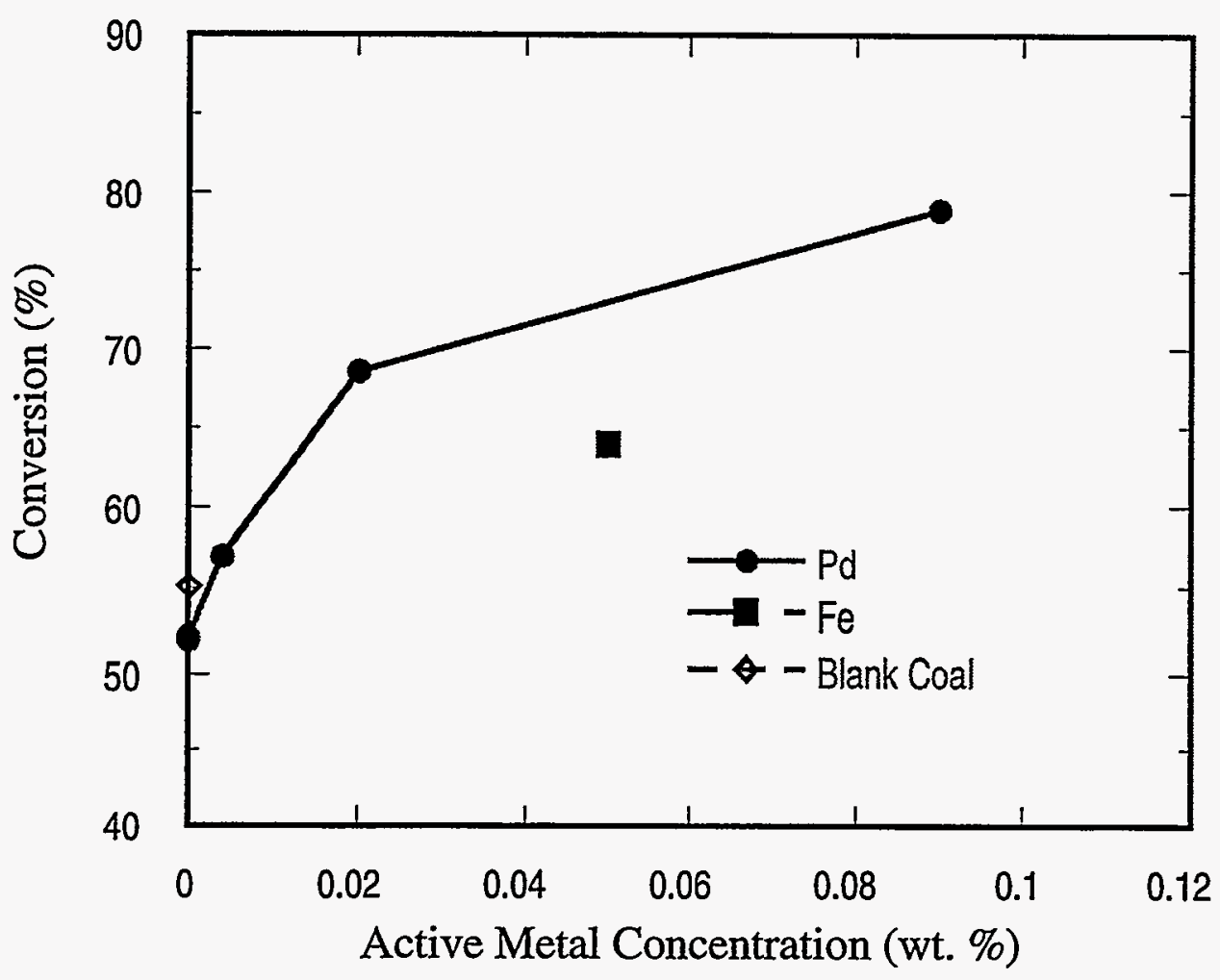

Figure 6. Herrin Burning Star No. 2 hydropyrolysis results for PdII, FeI, and a commercial $\mathrm{MoS}_{2}$ catalyst. 


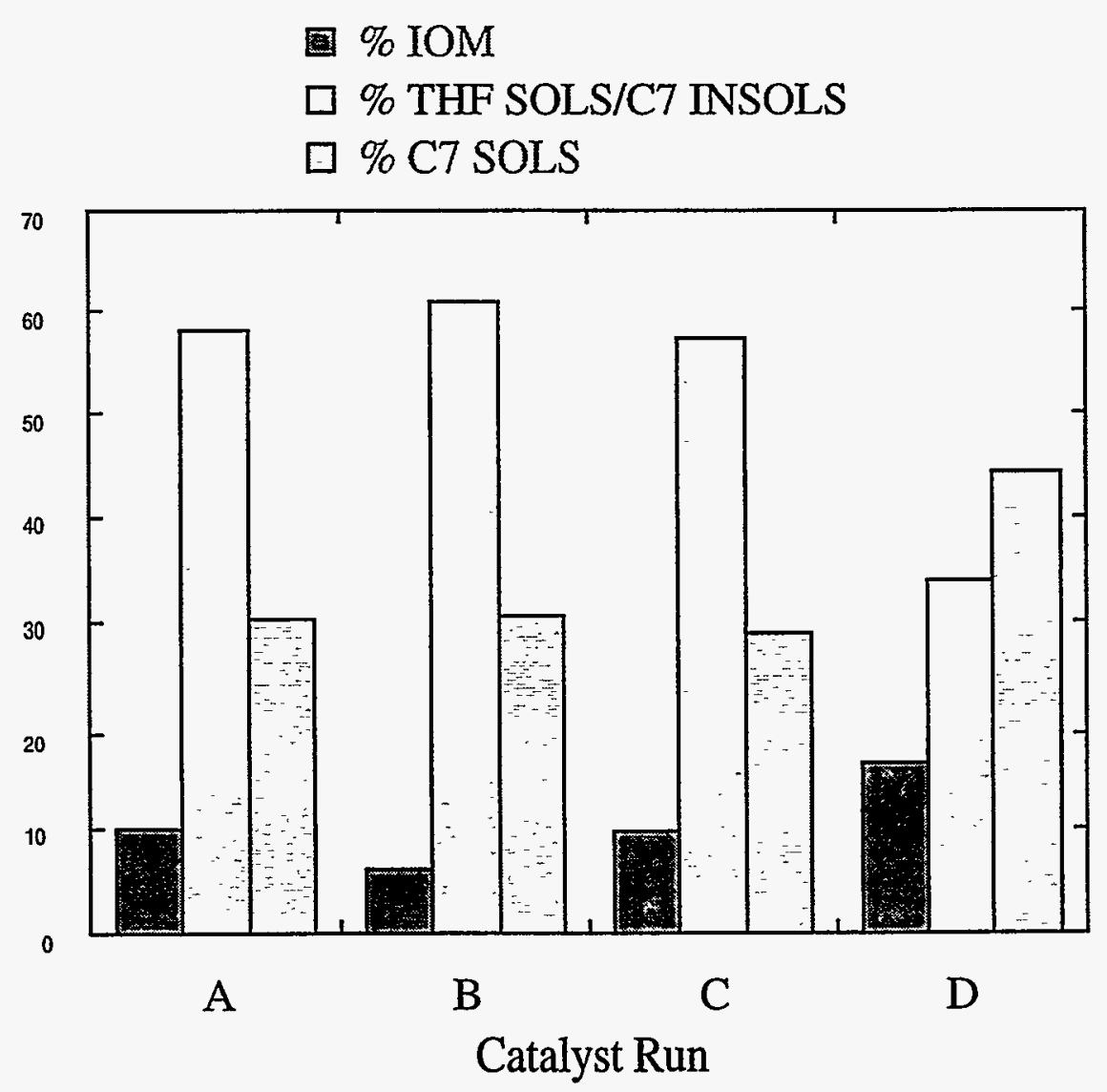

Figure 7. DECS-17 Blind Canyon coal liquefaction results for Fe based clusters. $A=$ thermal average; $\mathrm{B}=\mathrm{FeIV}\left(\sim 2 \%\right.$ active metal basis); $\mathrm{C}=\mathrm{FeS}_{2} \mathbf{I I I}(\sim 1.2 \%$ active metal basis $) ; \mathrm{D}=$ $\mathrm{FeS}_{2} \mathrm{IV}(\sim 2.4 \%$ active metal basis). 


\title{
Chapter 2
}

\section{The Synthesis and Characterization of Iron Colloid Catalysts in Inverse Micelle Solutions}

\begin{abstract}
We have studied the synthesis and characterization of Fe based nanometer sized clusters formed in inverse micelle solutions. Inverse micelles provide a colloidal sized reaction template in an oleic solvent. Metal salts are solubilized within the interior of inverse micelles, and the addition of a reducing agent initiates nucleation and growth to produce the clusters. Surfactant acts to stabilize the particles. TEM shows that the average particle size is less than $3 \mathrm{~nm}$ in diameter. The reduction reaction of iron salts with $\mathrm{LiBH}_{4}$ in inverse micelle solutions results in the formation of $\mathrm{FeB}, \mathrm{Fe}^{2+}{ }^{+} \mathrm{BO}_{\mathrm{X}}$ (i.e. pyroborate), and $\alpha$ - $\mathrm{Fe}$ as determined by Mossbauer spectroscopy, electron diffraction, and x-ray photoelectron spectroscopy (XPS). Oxidation after exposure to air leads to the conversion of the FeB to $\alpha$ $\mathrm{Fe}$ then to an undetermined $\mathrm{Fe}^{2+}$ phase and then finally to $\mathrm{Fe}_{3} \mathrm{O}_{4}$. Iron based metals are of interest as active, selective catalysts for a number of hydrogenation reactions including methanation and Fischer-Tropsch synthesis. We report catalytic results of iron clusters in the hydrogenolysis of naphthyl bibenzyl methane (NBM), a model reaction for coal liquefaction. The role of surfactant in the reaction mechanism is determined.
\end{abstract}

\section{Introduction}

Colloidal sized metal and semiconductor particles (1 - $20 \mathrm{~nm})$ are of current interest, because they mark a material transition range between molecular and bulk properties. With decreasing colloid size, bulk properties are lost as the continuum of electronic states breaks down (i.e. quantum size effects) and as the fraction of surface atoms becomes large. There are a number of ways to synthesize metal particles with diameters between 1-20 nm (nanoclusters) [1-3]. All synthesis routines include nucleation, growth, and stabilization of the particles and attempt to control particle size, size distribution, chemical composition, and structure. Chemical or photolytic reduction [4-6], thermal decomposition [7-9], and vapor 
phase condensation [10-12] of metal salts and organometallic reagents initiates nucleation and growth. Controlling double layer forces with buffers and electrolytes [13,14] and the use of steric stabilizing agents such as polymers [15-24], surfactants [25-33], and bulky ligand appendages [34-41] stabilize the particles in solution.

A common nanocluster synthesis technique is the reduction of metal salts in inverse micelle solutions [25-33]. Inverse micelles are solution structures formed by the selfassembly of surfactants in apolar solvents (i.e. toluene, alkanes). Surfactants possess two distinct moieties, a hydrophilic head group and a hydrophobic tail group, and they selfassemble in apolar solvents so that the hydrophilic head groups shield themselves from the oleic surroundings [42]. The relatively polar head group regions solubilize and confine added metal salts and act as reaction cages when a reducing agent is introduced. The reaction sequence is sustained by material exchange between inverse micelles, and final particle size is dependent on stabilization by the surfactant. Ultra-small, monodispersed particles sterically stabilized in solution by surfactant are formed.

There are numerous advantages to synthesizing colloidal sized metal particles in inverse micelle solutions. The inverse micelle structure provides a reaction template in the appropriate size range, and size control is achieved by regulating the nucleation and growth process [2]. The size of particles formed by the reduction of metal salts in inverse micelle solutions depends on the inherent size of the inverse micelles and the material exchange rate between inverse micelles. Both micelle size and the material exchange rate depend on experimental parameters like the surfactant/solvent system, the addition of water which swells the inverse micelles, and the salt to surfactant ratio. The inverse micelle technique provides a single synthesis route to producing a number of different metals, metal oxides, and metal sulfides. Metal type is dependent only on the initial metal salt chosen, and $\mathrm{Au}, \mathrm{Ag}, \mathrm{Pt}$, $\mathrm{Pd}, \mathrm{Rh}$, and $\mathrm{Ni}$ and $\mathrm{Fe}$ based metals are examples of different colloids synthesized in inverse micelles [43-47]. Bimetallic colloids are formed by starting with mixtures of metal salts. Metal sulfides are synthesized by using sulfiding agents rather than reducing agents [48-61], and metal oxides are produced by using reducing agents in a basic environment [62-64].

We have explored the synthesis of iron based nanoclusters in inverse micelles. Characterization of the size of the clusters is completed with transmission electron microscopy (TEM), and chemical composition of the clusters synthesized by an Fe salt, borohydrid reduction reaction is completed with Mossbauer spectroscopy, electron 
diffraction, and x-ray photoelectron spectroscopy (XPS). The effect of oxidation on the clusters is determined. Our interest in iron colloids stem from their potential use as coal liquefaction catalysts [79-81]. Iron based metals are also of interest as active, selective catalysts for a number of hydrogenation reactions including methanation and Fischer-Tropsch synthesis $[82,83]$. Here, we test the iron clusters in the hydrogenolysis of naphthyl bibenzyl methane (NBM), a model reaction for coal liquefaction.

Metal clusters prepared by a number of techniques have been studied as potential catalysts [68-71]. Reactant adsorption and the reactivity in various processes depend strongly on particle size. $\mathrm{Pt}, \mathrm{Pd}, \mathrm{Rh}$, and Ir particles prepared via inverse micelle techniques [72] have been studied in the hydrogenation and isomerization of but-1-ene [73,74], and the hydrogenolysis and isomerization of hexanes [75]. The activity of these catalysts increases when supported, and the selectivity of the colloidal sized particles is a function of the particle size. These studies found that activity is hindered by the presence of surfactant most likely due to steric effects on the metal surface. In other studies, the presence of surfactant on' $\mathrm{Ni}_{2} \mathrm{~B}$ enhances activity by increasing the dispersion of the particles despite steric effects, but adsorbed species on $\mathrm{Co}_{2} \mathrm{~B}$ particles decrease activity again most likely due to steric effects [76]. In catalyst tests performed on colloids in normal micelles, the presence of surfactant enhances selectivity $[77,78]$ as hydrogenation is preferred when the reactant mimics the surfactant and the double bond is accessible to the metal surface.

\section{Experimental}

Octane and toluene were purchased from Aldrich at $99.9+\%$ purity. Surfactants used include didodecyldimethylammonium bromide (DDAB) from Kodak and butyl-ethylene glycol n-dodecyl ether $\left(\mathrm{C}_{12} \mathrm{E}_{4}\right)$ from Nikkol. Metal salts used include iron(III) chloride hexahydrate from Aldrich and iron(II) tetrafluoroborate hexahydrate from Alfa Chemicals. Lithium borohydride in tetrahydrofuran (2M) was purchased from Aldrich.

A. Synthesis of Fe Based Colloids: Four different iron samples (FeI, FeII, FeIII, and FeIV) were prepared and studied (Table 1). The reactions and all chemical manipulations are carried out in a glove box under dry, oxygen-free conditions. The synthesis technique is general and specific samples differ only in the type and concentration of salts and surfactant added to the apolar solvents. First, the inverse micelle solutions are prepared by adding 
surfactant to the solvents (i.e. DDAB in toluene, $\mathrm{C}_{12} \mathrm{E}_{4}$ in octane). Then, the metal salts are introduced and the precursor salt solutions are mixed overnight on a stirring plate to assure complete solubilization. Transparent yellow Fe salt solutions are formed. $2 \mathrm{M} \mathrm{LiBH}_{4} / \mathrm{THF}$ solution is directly injected into the salt solutions under rapid stirring to initiate the reduction of iron. The reaction is run at a 3:1 molar ratio of $\left[\mathrm{BH}_{4}^{-}\right]:\left[\mathrm{Fe}^{2+}\right]$. The reacted Fe solutions turn black upon reduction, and the colloids formed in solution are stabilized by surfactant indefinitely. As an example of the synthesis, Fel is made by first mixing $10 \mathrm{wt}$. \% DDAB in toluene. $0.01 \mathrm{M} \mathrm{FeCl}_{3}$ is added to the solution and mixed overnight. The $2 \mathrm{M} \mathrm{LiBH}_{4}$ in THF is then directly injected to total $0.03 \mathrm{M} \mathrm{LiBH}_{4}$ in the Fe salt, inverse micelle solution.

Further manipulation of the DDAB/toluene solutions produces iron powders. When approximately $15 \mathrm{vol} . \%$ methanol is added to the colloidal solution, the surfactant is washed from the colloid surface, and the particles aggregate. A black precipitate forms below a clear and transparent solution after several hours. The precipitate is separated from the solution by centrifugation and washed with methanol. The process is repeated two or three times, and the powder is finally dried (FeIl). We have also coated the particles with poly(vinylpyrrolidone) ( $M W=40,000)$ by adding the polymer and iron powder to methanol. The particles remain in solution for days without the presence of surfactant. Removing the methanol produces Fe particles embedded in PVP powder (FeIV). Replacing surfactant with polymer allows us to control the ratio of stabilizing agent to metal, remove the relatively volatile surfactant for $\mathrm{x}$-ray photoelectron spectroscopy studies, and protect the particles from oxidation during air exposure.

B. Characterization of the Colloids: Particle size and composition in the colloidal solutions are characterized with transmission electron microscopy (TEM) and electron diffraction. These tests are performed with a Jeol 1200EX electron microscope. The colloidal solutions are applied directly on a holey carbon grid, and the solution is wicked away by adsorbent towels under the grid leaving the particles on the grid. Transfer of the particle coated grid into the microscope briefly exposes the sample to air.

Mossbauer spectroscopy is used to determine the chemical state of the iron in-situ (FeII) and in the PVP coated iron powders (FeIV). The Mossbauer Spectrometer (Austin Science) and its operation are described in detail elsewhere [84]. The spectra are obtained using a $60 \mathrm{mCi}^{57} \mathrm{Co}$ in palladium foil source and computer fitted to Lorentzian lines with a 
least-squares optimization procedure. The spectra are corrected during the collection procedure to remove the curved background of instrumental origin, and resonant absorption areas are found from integration of the background curvature-corrected spectra. With the use of an absolute laser velocity calibrator it is possible to measure isomer shifts to within an absolute accuracy of $+1-0.005 \mathrm{~mm} / \mathrm{s}$. The in-situ sample was tested in the frozen state $(\mathrm{T}=-$ $176^{\circ} \mathrm{C}$ ), and the particle embedded polymer powder was tested under four conditions: at room temperature without air exposure, at room temperature after air exposure, at $-183^{\circ} \mathrm{C}$, and at $-183^{\circ} \mathrm{C}$ with an applied magnetic field of $11 \mathrm{kGauss}$.

$\mathrm{X}$-ray photoelectron spectroscopy (XPS) is used to determine the chemical state of the iron particles. XPS is performed with a VG Microtech Clam2 analyzer operating at a resolution of $1 \mathrm{eV}$ and a $600 \mathrm{~W} \mathrm{Al} \mathrm{K} \alpha \mathrm{X}$-ray source. Assignment of the iron oxidation state relies on comparison with the $\mathrm{Fe} 2 \mathrm{p}$ peak positions and lineshapes of iron metal and the various iron oxides [85]. These standard lineshapes have been verified on the same instrument used to analyze the colloidal iron particles. Charge referencing was performed for all samples by adjusting the $C 1 \mathrm{~s}$ binding energy to $284.6 \mathrm{eV}$. To prepare samples of the surfactant stabilized iron particles suitable for XPS analysis, the in-situ solution (FeII) was evaporated to concentrate the particles and a drop of the resulting solution was placed on a $\mathrm{Ta}$ foil and allowed to evaporate completely overnight. For analysis of the washed iron powders (FeIII), the particles were re-dispersed in methanol and a drop of the dispersed solution was placed on a Ta foil and again allowed to dry. Finally, for the particles embedded in PVP (FeIV), the PVP matrix was dissolved in methanol and a drop of the resulting solution was placed on a Ta foil and the methanol evaporated to form a PVP film. All samples were prepared and mounted on a sample holder inside a dry, oxygen free glove box. As with microscopy, transfer of the sample to the XPS chamber requires brief exposure to air.

Complexometric experiments were used to determine the extent of reaction of the iron salts upon reduction. 1, 10 phenanthroline forms a complex with ionic $\mathrm{Fe}^{2+}$ species that has a distinctive maximum at $530 \mathrm{~nm}$. The peak is followed before and after reaction with UVvisible spectrophotometry (Hewlett-Packard 8452A diode array spectrophotometer) by adding 1,10 phenanthroline to the Fe salt precursor solutions and reacted Fe colloid solutions. The quantity and nature of the $\mathrm{Fe}^{2+}$ species present is determined. Two salt solutions were studied: $\mathrm{FeCl}_{3}$ and $\mathrm{Fe}\left(\mathrm{BF}_{4}\right)_{2}$ in 10 wt. \% $\mathrm{C}_{12} \mathrm{E}_{4} /$ octane. 
Finally, BET surface area analysis of the methanol washed iron powder was carried out with a Quantachrome Autosorb-6 surface analysis apparatus.

C. Naphthyl Bibenzyl Methane (NBM) Hydrogenolysis: Hydrogenolysis testing of $\mathrm{NBM}$ has been developed as a model reaction for coal liquefaction [86]. We add $100 \mathrm{mg}$ of NBM, $400 \mathrm{mg}$ of the hydrogen donating solvent 9,10 dihydrophenanthrene $(9,10 \mathrm{DHP})$, and up to 15 wt. \% catalyst on an NBM basis to a $10 \mathrm{ml}$ flame sealed glass test tube. If used, 15 $\mathrm{mg}$ of elemental sulfur is added. The test tube is sealed under ambient air pressure. The tube is placed in a $400^{\circ} \mathrm{C}$ sand bath for one hour. Previously, NBM hydrogenolysis has been used to study the selectivity of catalysts for five different cleavage reactions. We do not quantify the selectivity of the catalysts used here. Conversion is calculated as the ratio of the molar sum of all products to the initial amount of NBM. The products are worked up in THF and analyzed by gas chromatography. Methanol washed Fe powders were tested as catalysts (FeII). These results were compared to the commercial catalyst Shell 324 (12.4 wt. \% Mo, 2.8 wt. \% $\mathrm{Ni}$ on $\mathrm{Al}_{2} \mathrm{O}_{3}$ ).

\section{Results}

Transmission electron micrographs show that the product of the reaction is highly dispersed nanometer sized particles of uniform size and shape (Figure 1). The number average diameter by TEM is $1.5+/-0.2 \mathrm{~nm}$ (FeI). Fe particle size as determined by TEM shows no discernible trend and is roughly constant in the ranges of $0.001 \mathrm{M}-0.01 \mathrm{M} \mathrm{FeCl} 3$ salt concentration and 1-10 wt. \% DDAB in toluene. In this range, the average particle diameter is $2.4 \mathrm{~nm}$. The methanol extracted Fe powder (FeII) characterized by TEM and BET surface analysis consists of agglomerated particles. Large agglomeration structures larger than $100 \mathrm{~nm}$ in size are observed (Figure 2). These structures consist of the ultra small particles (approximately $2 \mathrm{~nm}$ in diameter) flocculated together. Multi-point BET analysis gives a surface area of $156 \mathrm{~m}^{2} / \mathrm{gm}$ for this sample. The degree of re-dispersion upon addition of PVP has not been determined. As the particles are stable in solution for only a couple of days with PVP, it is likely that the degree of dispersion is not as great as after the original reaction in the presence of surfactant.

Mossbauer spectroscopy completed on the in-situ sample (Fell) frozen at $-176^{\circ} \mathrm{C}$ (Figure 3) indicates the presence of three different forms of iron. The spectrum is fit with 
two doublets and a singlet (corresponding Mossbauer parameters are found in Table 2). We assign the first doublet to the presence of fine superparamagnetic particles of $\mathrm{FeB}$, the second doublet to the presence of $\mathrm{Fe}^{2}+\mathrm{BO}_{\mathrm{X}}$, and the singlet to the presence of superparamagnetic $\mathrm{Fe}$ metal. On the basis of spectral areas, the iron phases are present at 83,15, and 2 mole \%, respectively with the species order listed above. The mole percentages are based on equal recoil-free fractions of each phase, and this assumption is only approximately correct in the case of small clusters.

Mossbauer spectroscopy completed on the particle embedded polymer powder (FeIV) indicates the presence of the same three forms of iron. The spectrum for the powder at $21^{\circ} \mathrm{C}$ before exposure to air (Figure 4) is fit with two doublets and a singlet (corresponding Mossbauer parameters are found in Table 3). The mole $\%$ of $\mathrm{FeB}, \mathrm{Fe}^{2}+\mathrm{BO}_{\mathrm{X}}$, and $\mathrm{Fe}$ metal are 60,10 , and 30 mole $\%$, respectively. There is no discernible change in the spectrum or fit after the sample is exposed to air. The spectrum of the sample at $-183^{\circ} \mathrm{C}$ is quite different from the room temperature spectrum. It appears that the two large peaks in the room temperature spectrum have broadened. The broadened spectrum is fit with a sextet and two doublets. The sextet has a magnetic hyperfine splitting of $128 \mathrm{kOe}$ and an isomer shift of $0.35 \mathrm{~mm} / \mathrm{sec}$ consistent with the presence of superparamagnetic FeB. When a magnetic field is applied to the sample, the spectrum is further broadened.

Selected area electron diffraction patterns of in-situ iron particles (FeII) deposited on the microscope grid are consistent with the presence of $\alpha$-Fe. Diffraction parameters, $d$, from scattering patterns depend on instrument settings and the radius of each scattering maxima (d $=L \lambda / R$, where $L$ is the instrument sample to detector distance, $\lambda$ is the electron beam wavelength, and $\mathrm{R}$ is the diffraction ring radius). The ratios of theoretical diffraction parameters to the first diffraction parameter $\left(\mathrm{d}_{\mathrm{d}} \mathrm{d}_{1}\right)$ are known for many crystalline materials. The diffraction pattern of FeIl shows several maxima, and experimentally we observe $\mathrm{d} / \mathrm{d}_{1}$ equal to $1,0.701,0.573,0.482,0.436$, and 0.381 . Theoretical values of $d / d_{1}$ for $\alpha$-Fe equal 1 , $0.707,0.577,0.500,0.447$, and 0.408 . Theoretical values of $d / d_{1}$ for FeB equal $1,0.842$, $0.728,0.696,0.670$, and 0.615 . The electron diffraction results, therefore, indicate the presence of $\alpha-\mathrm{Fe}$ and do not indicate the presence of any crystalline iron oxides, borides, or 
borates. A separate diffraction pattern consistent with the presence of crystalline $\mathrm{B}_{2} \mathrm{O}_{3}$ as a reaction by-product is also detected.

XPS detects $\mathrm{Fe}^{2+}$ for both the iron particles embedded in PVP (FeIV) and the in-situ iron particles (FeII). For FeIV, an Fe 2p3/2 binding energy of $\sim 709.0 \mathrm{eV}$ and a prominent shakeup feature at $\sim 715 \mathrm{eV}$ is observed (Figure 5a). Zero valent Fe either as a metal or boride is not detected. The signal to noise ratios for these spectra are poor due to the presence of either surfactant or PVP near the sample surface. The low signal to noise ratios prevent the detection of boron in these samples and prevent the possible detection of small amounts of zero valent $\mathrm{Fe}$, which would appear at $707.0 \mathrm{eV}$. Signal to noise ratios are improved slightly by mild heating in vacuum, but not enough to allow detection of boron or positive identification of a metallic iron component. For the uncoated iron particles (FeIII), the signal to noise ratio is an order of magnitude higher, and the initial state of the iron is clearly a mixture of $\mathrm{Fe}^{2+}$ and $\mathrm{Fe}^{3+}$ (Figure $5 \mathrm{~b}$ ). The resulting spectrum is quite similar to that of magnetite $\left(\mathrm{Fe}_{3} \mathrm{O}_{4}\right)$, and no more than $2-3 \%$ of the detected iron is present as metallic iron or iron boride. An oxidized form of boron is also detected with a $B$ 1s binding energy of $192.0 \mathrm{eV}$ consistent with the presence of either $\mathrm{B}_{2} \mathrm{O}_{3}$ or an iron borate. Heating the washed iron particles to $300^{\circ} \mathrm{C}$ under vacuum results in elimination of the $\mathrm{Fe}^{3+}$ contribution and the emergence of a pure $\mathrm{Fe}^{2+}$ spectrum.

Complexometric experiments indicate that the reaction is complete upon the addition of $\mathrm{LiBH}_{4}$. In the $\mathrm{Fe}\left(\mathrm{BF}_{4}\right)_{2}$ precursor solutions, the $\mathrm{Fe}^{2+}$ species complexes with 1,10 phenanthroline, and the distinctive peak is observed at $530 \mathrm{~nm}$. The peak is non existent in $\mathrm{Fe}\left(\mathrm{BF}_{4}\right)_{2}$ samples after the addition of $\mathrm{LiBH}_{4}$ indicating the reaction goes to completion. In $\mathrm{FeCl} 3$ precursor solutions, no complex is formed with the $\mathrm{Fe}^{3+}$ species. After reaction, a slight peak at $530 \mathrm{~nm}$ is detected indicating some conversion of $\mathrm{Fe}^{3+}$ to $\mathrm{Fe}^{2+}$ but too little to quantify.

Hydrogenolysis of naphthyl bibenzyl methane (NBM) was tested with several inverse micelle synthesized iron based catalysts and compared with the commercial catalyst Shell 324. We tested the methanol extracted Fe powder (FeIII) with and without sulfur additives and the dispersed catalysts with surfactant (FeI). In each experiment we monitored the percentage recovery of the reactant NBM and the percentage conversion to products by gas 
chromatography. In each experiment we also monitored the percentage recovery of the hydrogen donating solvent 9,10 DHP and the percentage converted to its dehydrogenated form phenanthrene. In an average of six runs, NBM was recovered at nearly $97+/-3 \%$ in thermal runs (no catalyst) and no product was obtained (Figure 6a). Some NBM was converted to products in thermal runs with added elemental sulfur. Little 9,10 DHP was dehydrogenated to phenanthrene in the thermal runs. FeIII as catalyst decreases the amount of NBM recovered to $87 \%$ and with added $S$ to $75 \%$, and product recovery correspondingly increases. Hydrogens are given up to the hydrogenolysis products, and some 9, $10 \mathrm{DHP}$ is dehydrogenated to phenanthrene (Figure 6b). The dispersed catalysts with surfactant present (FeI) showed little activity compared to thermal runs.

Much higher product yields were obtained with Shell 324 (Figure 7). Higher conversions of 9,10 DHP to phenanthrene are consistent with these higher yields. In order to study the effect of surfactant on catalytic activity, we doped Shell 324 with increasing amounts of DDAB. As the Shell 324:DDAB mass ratio decreases, the trends in reactant recovery and product conversion move back to the thermal results indicating loss of catalytic activity. Initially the conversion of 9,10 DHP to phenanthrene moves toward the thermal results as well. At the lower Shell 324:DDAB ratios, however, this trend reverses itself and nearly all of the $9,10 \mathrm{DHP}$ is converted to phenanthrene.

\section{Discussion}

Introduction of $\mathrm{LiBH}_{4}$ to iron salt containing inverse micelle solutions causes immediate reduction of the salt to form nanometer sized, highly dispersed particles of equal size and shape. The reaction goes to completion. No initial Fe salt remains as determined by the complexometric experiments. $\mathrm{LiBH}_{4}$ enters the surfactant-oil solution and is solubilized within the inverse micelle structure. The reaction is initiated and is sustained through material exchange between inverse micelles. After the particle growth has equilibrated, the surfactant stabilizes the particles and prevents flocculation and precipitation. The final particle size of nanometer sized clusters formed in inverse micelles depends on a complicated nucleation and growth process [2]. Inverse micelles effect this process primarily in two ways: (1) diffusion of the reacting nucleation sites and ions is governed by exchange rates between micelles and (2) the critical nucleation size depends inherently on the size of the inverse micelles. Size dependence of metal borides, $\mathrm{CdS}$, and $\mathrm{CdSe}$ clusters produced in 
inverse micelles has been discussed previously [48-61]. The Fe particles synthesized in DDAB/toluene mixtures showed no size dependence by TEM on the salt to surfactant concentration ratio within the range studied.

Mossbauer spectroscopy of in-situ Fe colloids (FeII) indicates three products are formed by the reduction of iron salts with $\mathrm{LiBH}_{4}$ in inverse micelle solutions. FeB, $\mathrm{Fe}^{2+} \mathrm{BO}_{\mathrm{x}}$, and metallic $\mathrm{Fe}$ are synthesized to 83 mole $\%, 15$ mole $\%$, and 2 mole $\%$, respectively. Similar type spectra were observed for the $\mathrm{NaBH}_{4}$ reduction of Fe salts in diglyme [82], and the doublet was assigned to the presence of FeB. The isomer shifts we report are higher, but they are consistent with those reported by Shinjo et al. [88]. Metallic Fe produces a singlet with an isomer shift close to zero $[89,90]$. Clearly, $\square-\mathrm{Fe}$ is not the majority phase as determined by Mossbauer. The following reaction accounts for the presence of the three products: $3 \mathrm{Fe}^{2+}+4 \mathrm{BH}_{4}^{-}+\mathrm{xH}_{2} \mathrm{O} \rightarrow-->\mathrm{FeB}+\mathrm{Fe}(\mathrm{s})+\mathrm{Fe}^{2+} \mathrm{BO}_{\mathrm{X}}+(5$ $+\mathrm{x}) \mathrm{H}_{2}+\mathrm{B}_{2} \mathrm{H}_{6}$. The waters of hydration of the metal salt provide the source of water, and pyroborate, $\mathrm{Fe}_{2} \mathrm{~B}_{2} \mathrm{O}_{3}$, is one possible $\mathrm{Fe}^{2+}$ borate phase represented by $\mathrm{Fe}^{2+} \mathrm{BO}_{\mathrm{x}}$. Similar reaction products and a similar reaction mechanism are found for the borohydride reduction of iron salts in a nonaqueous solvent [91, 92]. Mossbauer spectroscopy on the particle embedded polymer powder ( $\mathrm{FeIV}$ ) detects the three phases, $\mathrm{FeB}, \mathrm{Fe}^{2+}{ }_{\mathrm{BO}}$, and metallic $\mathrm{Fe}$, in approximate mole percentages of $60 \%, 10 \%$, and $30 \%$, respectively. The conversion of approximately $25 \%$ of $\mathrm{FeB}$ to $\mathrm{Fe}(\mathrm{s})$ results from the methanol workup necessary to extract the particles from solution and prepare the powder. There is no discernible change in the spectra or fit after the powder is exposed to air. The iron is apparently protected from oxidation by the polymer.

Mossbauer results of the powder at low temperatures and in the presence of a magnetic field further support the presence of $\mathrm{FeB}$. The spectral broadening is consistent with longer magnetic relaxation times in a superparamagnetic species, and the sextet has a hyperfine field consistent with $\mathrm{FeB}$. In the limit of a sufficiently large magnetic field or at sufficiently low temperatures, the spectrum would resolve into six lines.

Electron diffraction of the in-situ Fe colloids (FeII) gives strong evidence for the presence of $\alpha-\mathrm{Fe}$. FeB is not detected. It has been reported that the presence of air during the reduction process favors the formation of metallic metal over metal borides by the 
following mechanism: $\mathrm{MB}+\mathrm{O}_{2}--->\mathrm{M}(\mathrm{s})+\mathrm{B}_{2} \mathrm{O}_{3}[91,92]$. In our procedure, the reaction is done in an argon environment, but the TEM grid is exposed to air prior to microscopy. The above stated mechanism is most likely responsible for the detection of $\alpha$-Fe as the majority phase by electron diffraction while $\mathrm{FeB}$ is the majority phase detected by Mossbauer. The detection of crystalline $\mathrm{B}_{2} \mathrm{O}_{3}$ by diffraction supports this theory.

XPS of the in-situ solution (FeII) and the polymer coated particles (FeIV) detects an undetermined $\mathrm{Fe}^{2+}$ species. We postulate that the $\mathrm{Fe}^{0}$ particles $(\mathrm{FeB}$ and $\alpha-\mathrm{Fe}$ ) formed by the reduction reaction and the oxidation of $F e B$ undergo further surface oxidation to form a thin, poorly crystalline oxide layer over an $\mathrm{Fe}^{0}$ core. The presence of metallic iron detected by Mossbauer is most likely obscured by the oxide layer and the poor signal to noise ratio. The surface oxidation does not continue on to the formation of $\mathrm{Fe}_{3} \mathrm{O}_{4}$ as in bulk iron samples [87]. The small size of the iron particles (in the case of FeII) or the small grain sizes (in the case of FeIV) and the presence of the surfactant or polymer may result in oxidation behavior that differs from bulk iron. XPS of the iron powder (Fem) indicates the presence of $\mathrm{Fe}_{3} \mathrm{O}_{4}$ or a mixture of $\mathrm{Fe}^{2+}$ and $\mathrm{Fe}^{3+}$. Since the washed sample is severely agglomerated and not protected by either surfactant or polymer, it is not surprising that the oxidation behavior upon exposure to air is similar to the oxidation behavior of bulk iron metal. Also, XPS detection of an oxidized boron species (not a boride, however) in the washed sample is consistent with the conversion of $\mathrm{FeB}$ into iron metal and $\mathrm{B}_{2} \mathrm{O}_{3}$ upon air exposure. Conversion of $\mathrm{Fe}^{3+}$ to $\mathrm{Fe}^{2+}$ upon heating $\mathrm{FeIII}$ to $300^{\circ} \mathrm{C}$ under vacuum suggests that the $\mathrm{Fe}^{3+}$ is a superficial surface layer formed during brief air exposure during sample loading.

Hydrogenolysis of NBM indicates catalytic activity with FeIII. A more than linear increase in activity with added $\mathrm{S}$ indicates that there is a synergistic effect between the $\mathrm{Fe}$ and S. Catalyst activity of Shell 324 far exceeds the activity of the iron based clusters. Doping Shell 324 with surfactant illustrates the effect of surfactant on catalyst activity. When Shell 324 is doped with surfactant, catalyst activity decreases (Figure 7). The behavior of the hydrogen donating solvent indicates two mechanisms are at work. At low surfactant concentrations, a decrease in product conversion and a decrease in the amount of $9,10 \mathrm{DHP}$ dehydrogenated to phenanthrene indicates less activity due to a chemical or steric poisoning of the catalyst. At high surfactant concentrations, we propose that a decrease in NBM 
conversion and an increase in the amount of 9, 10 DHP dehydrogenated to phenanthrene indicates that the thermal pyrolysis products of the surfactant (observed by GC) scavenge the hydrogens from the donor solvent. The dispersed catalyst (FeI) showed little activity most likely due to the presence of surfactant. The apparent advantage of the nanometer size of the particles is eliminated by the surfactant.

\section{Conclusions}

We have synthesized nanometer sized, highly dispersed $\mathrm{Fe}$ based particles in inverse micelle systems. TEM shows that the average particle size is less than $3 \mathrm{~nm}$ in diameter. Mossbauer spectroscopy confirms that the products of the reduction reaction of iron salts with $\mathrm{LiBH}_{4}$ are $83 \% \mathrm{FeB}, 15 \% \mathrm{Fe}^{2}+\mathrm{BO}_{\mathrm{X}}$, and $2 \% \mathrm{Fe}^{0}$. Methanol washing and forced precipitation causes uncontrolled growth of the particles and results in relatively high surface area powders. $\mathrm{FeB}$ is converted to $\alpha-\mathrm{Fe}$ then to an undetermined $\mathrm{Fe}^{2+}$ phase and then finally to $\mathrm{Fe}_{3} \mathrm{O}_{4}$ as the degree of oxidation by exposure to air increases. Exposure to methanol converts approximately $25 \%$ of $\mathrm{FeB}$ to $\mathrm{Fe}^{0}$. A shell and core picture of the clusters develops with oxidation as detected by XPS. Catalytic activity of the ultra-small particles is determined in a model compound hydrogenolysis reaction. When surfactant is present, catalytic activity is hindered by two mechanisms. Surfactant chemically poisons the catalyst, and by-products of surfactant disintegration at reaction conditions scavenge hydrogen from hydrogen donating solvents. This latter mechanism is important in coal liquefaction as this process depends strongly on hydrogen transfer.

\section{Acknowledgments}

This work is supported by the U.S. Department of Energy under contract DE-AC0494AL85000. 


\section{References}

(1) J.S. Bradley, "Clusters and Colloids, From Theory to Applications", Ed. G. Schmid, VCH Publications, Inc., 1994, Chapter 6.

(2) M.L. Steigerwald and L.E. Brus, Annu. Rev. Mater. Sci., 19 (1989) 471.

(3) A. Henglein, Chem. Rev., 89 (1989) 1861.

(4) J. Shen, Z. Hu, Y.Hsia, and Y. Chen, Appl. Phys. Lett., 59 (1991) 2510.

(5) K. Takiyama, Bull. Chem. Soc. Jpn., 31 (1958) 944.

(6) J. Turkevich, P.C. Stevenson, and, J. Hillier, J. Am. Chem. Soc. Faraday Soc. Disc., 11 (1951) 55.

(7) J.G. Brennan, T. Siegrist, P.J. Carroll, S.M. Stuczynski, L.E. Brus, and M.L. Steigerwald, J. Am. Chem. Soc., 111 (1989) 4141.

(8) S.R. Hoon, M. Kilner, G.J. Russell, and B.K. Tanner, J. of Magnetism \& Magnetic Materials, 39 (1983) 107.

(9) C.H. Griffiths, M.P. O'Horo, and T.W. Smith, J. Appl. Phys., 50 (1979) 7108.

(10) S.T. Lin, M.T. Franklin, and K.J. Klabunde, Langmuir, 2 (1986) 259.

(11) K. Kimura and S. Bandow, Bull. Chem. Soc. Jpn., 56 (1983) 3578.

(12) C.G. Francis, H. Huber, and G.A. Ozin, Inorg. Chem., 19 (1980) 219.

(13) M. Quinn and G. Mills, J. Phys. Chem., 98 (1994) 9840.

(14) G.L. Trivino, K.J. Klabunde, and E.B. Dale, Langmuir, 3 (1987) 986.

(15) Y. Ng Cheong Chan, R.R. Schrock, and R.E. Cohen, Chem. Mater., 4 (1992) 24.

(16) C.C. Cummins, R.R. Schrock, and R.E. Cohen, Chem. Mater., 4 (1992) 27.

(17) N. Ishizuki, K. Torigoe, K. Esumi, and K. Meguro, Colloids and Surfaces, 55 (1991) 15.

(18) W. Mahler, Inorg. Chem., 27 (1988) 435.

(19) Y. Wang, A. Suna, W. Mahler, and R.J. Rasowski, J. Chem. Phys., 87 (1987) 7315.

(20) J.P. Kuczynski, B.M. Milosavljevic, and J.K. Thomas, J. Phys. Chem., 88 (1984) 980.

(21) A.W.H. Mau, C.B. Huang, N. Kakuta, A.J. Bard, A. Campion, M.J. White, and S.E.

Webber, J. Am. Chem. Soc., 106 (1984) 6537.

(22) A. Fojtik, H. Weller, U. Koch, and A. Henglein, Ber. Bunsenges. Phys. Chem., 88 (1984) 969.

(23) D. Meisssner, D. Memming, and B. Kastening, Chem. Phys. Lett., 96 (1983) 34.

(24) P.H. Hess and P.H. Parker, J. Appl. Polym Sci., 10 (1966) 1915.

(25) K. Torigoe and K. Esumi, Langmuir, 8 (1992) 59.

(26) K. Esumi, M. Shiratori, H. Ishizuka, T. Tano, K. Torigoe, and K. Meguro, Langmuir, 7 (1991) 457.

(27) X.K. Zhao, S. Xu, and J.H. Fendler, Langmuir, 7 (1991) 520.

(28) E.C. O'Sullivan, R.C. Patel, and A.J.I Ward, J. Coll. Inter. Sci., 146 (1991) 582.

(29) C. Larpent, F. Brisse-Le Menn, and H. Patin, J. Molec. Catal., 65 (1991) L35.

(30) E. Borgarello, D. Lawless, N. Serpone, E. Pelizzetti, and D. Meisel, J. Phys. Chem., 94 (1990) 5048.

(31) K. Maguro, M. Torizuka, and K. Esumi, Bull. Chem. Soc. Jpn., 61 (1988) 341.

(32) K. Monserrat, M. Gratzel, and P. Tundo, J. Am. Chem. Soc., 102 (1980) 5527.

(33) J. Kiwi and M. Gratzel, J. Am. Chem. Soc., 101 (1979) 721.

(34) Y. Nosaka, N. Ohta, T. Fukuyama, and N. Fujii, J. Coll. Inter. Sci., 155 (1993) 23.

(35) Z. Duan, M.J. Hampden-Smith, A. Datye, P. Nigrey, C. Quintana, and A.P. Sylwester, J. of Catal., 139 (1993) 504.

(36) J.P. Folkers, P.E. Laibinis, and G.M. Whitesides, Langmuir, 8 (1992) 1330.

(37) F. Zimmermann and A. Wokann, Molec. Phys., 73 (1991) 959. 
(38) C. Becker, T. Fries, K. Wendelt, U. Kreibig, and G. Schmid, J. Vac. Sci. Technol., B 9 (1991) 810.

(39) D. Hayes, D. Meisel, and O.I. Micic, Collods and Surfaces, 55 (1990) 121.

(40) M.P. Andrews and G.A. Ozin, J. Phys. Chem., 90 (1986) 2929.

(41) A. Henglein, J. Phys. Chem., 83 (1979) 2209.

(42) D. Langevin, Acc. Chem. Res., 21 (1988) 255.

(43) J.P. Wilcoxon, R.L. Williamson, and R. Baughman, J. Chem. Phys., 98 (1993) 9933.

(44)P. Barnickel, A. Wokaun, Molec. Phys., 69 (1990) 1.

(45) J.B. Nagy, Colloids and Surfaces, 35 (1989) 201.

(46) J.B. Nagy, A. Gourgue, and E.G. Derouane, "Preparation of Catalysts II", Eds. G.

Poucelet, P. Grange, P.A. Jacobs, Elsevier Science Publishers, 1983, pg. 193.

(47) M. Boutonnet, J. Kizling, P. Stenius, and G. Maire, Colloids and Surfaces, 5 (1982) 209.

(48) L. Motte, C. Petit, L. Boulanger, P. Lixon, and M.P. Pileni, Langmuir, 8 (1992) 1049.

(49) M.P. Pileni, L. Motte, and C. Petit, Chem. Mater., 4 (1992) 338.

(50) B.H. Robinson, T.F. Towey, S. Zourab, A.J.W.G. Visser, and A. van Hoek, Colloids and Surfaces, 61 (1991) 175.

(51) C. Petit, P. Lixon, and M.P. Pileni, J. Phys. Chem., 94 (1990) 1598.

(52) A.R. Kortan, R. Hull, R.L. Opila, M.G. Bawendi, M.L. Steigerwald, P.J. Caroll, and

L.E. Brus, J. Am. Chem. Soc., 112 (1990) 1327.

(53) T.F. Towey, A. Khan-Lodhi, and B.H. Robinson, J. Chem. Soc. Faraday Trans., 86 (1990) 3757.

(54) S. Modes and P. Lianos, J. Phys. Chem., 93 (1989) 5854.

(55) C. Petit and M.P. Pileni, J. Phys. Chem., 92 (1988) 2282.

(56) M.L. Steigerwald, A.P. Alivisatos, J.M. Gibson, T.D. Harris, R. Korton, A.J. Muller, A.M. Thayer, T.M. Duncan, D.C. Douglass, and L.E. Brus, J. Am. Chem. Soc., 110 (1988) 3046.

(57) J.H. Fendler, Chem. Rev., 87 (1987) 877.

(58) P. Lianos and J.K. Thomas, Coll Surf. Sci., 117 (1986) 505.

(59) P. Lianos and J.K. Thomas, Chem. Phys. Lett., 125 (1986) 299.

(60) T. Dannhauser, M. O'Neil, K. Johansson, D. Whitten, and G. McLendon, J. Phys. Chem., 90 (1986) 6074.

(61) M. Meyer, C. Wallberg, K. Kurihara, and J.H. Fendler, J. Chem. Soc., Chem. Commun. 90 (1984).

(62) J.B. Nagy, A. Claerbout, "Surfactants in Solution" 11, Eds. K.L. Mittal, D.O. Shah, 1991 pg. 363.

(63) M. Gobe, K. Kon-No, K. Kandori, A. Kitahara, J. Coll. Inter. Sci., 93 (1983) 293.

(64) K. Kurihara, J.Kizling, P. Stenius, J.H. Fendler, J. Am. Chem. Soc., 105 (1983) 2574.

(68) L.N. Lewis, Chem. Rev., 93 (1993) 2693.

(69) H. Bonnemann, W. Brijoux, R. Brinkmann, E. Dinjus, T. Jouロen, B. Koral, Angew. Chem. Int. Ed. Engl., 30 (1991) 1312.

(70) S.C. Davis and K.J. Klabunde, Chem. Rev., 82 (1982) 153.

(71) Y. Sasson, A. Zoran, and J. Blum, J. Molec. Catal., 11 (1981) 293.

(72) M. Boutonnet, J. Kizling, P. Stenius, and G. Maire, Colloids Surf., 5 (1982) 209.

(73) M. Boutonnet, J. Kizling, V. Mintsa-Eya, A. Choplin, R. Touroude, G. Maire, and P. Stenius, J. of Catal., 103 (1987) 95.

(74) M. Boutonnet, J. Kizling, R. Touroude, G. Maire, and P. Stenius, Appl. Catal., 20 (1986) 163. 
(75) M. Boutonnet, J. Kizling, R. Touroude, G. Maire, and P. Stenius, Catal. Lett., 9 (1991) 347.

(76) J.B. Nagy, I. Bodart-Ravet, and E.G. Dorouane, J. Am. Chem. Soc., Faraday Disc., 87 (1989) 189.

(77) N. Toshima, T. Takahashi, and H. Hirai, Chem. Lett., (1985) 1245.

(78) M. Ohtaki, N. Toshima, M. Komiyama, and H. Hirai, Bull. Chem. Soc. Jpn., 63 (1990) 1433.

(79) D.W. Matson, J.L. Linehan, J.G. Darab, and M.F. Buehler, Energy \& Fuels, 8 (1994) 10.

(80) A. Martino, J.P. Wilcoxon, and J.S. Kawola, Energy \& Fuels, 8 (1994) 1289.

(81) T. Suzuki, H. Yamada, P.L. Sears, and Y. Watanabe, Energy \& Fuels, 3 (1989) 707.

(82) C. Wang and C.H. Bartholomew, Appl. Catal., 62 (1990) 221.

(83) H. Itoh, H. Hosaka, T. Ono, E. Kikuchi, Appl. Catal., 40 (1988) 53.

(84) L.R. Neubauer, Ph.D. Dissertation, Brigham Young University, 1986.

(85) C.S. Kuivila, J.B. Butt, and P.C. Stair, Appl. Surface Sci., 32 (1988) 99.

(86) M. Faracasiu and C. Smith, Prepr. Pap. Div. Fuel Chem.-Am. Chem. Soc. 35, 404,' 1990.

(87) A.G. Sault, Appl. Surface Sci., 74 (1994) 249.

(88) I.F. Shinjo, H. Takaki, Y. Nakamura, and N. Shikazono, J. Phys. Soc. Jpn., 19 (1964) 1252.

(89) P.A. Smith, Masters Thesis, Brigham Young University, 1990.

(90) J. Danon, "Chemical Applications of Mossbauer Spectroscopy", Eds. V.I. Goldanskii, R.H. Herber, Academic Press, 1968.

(91) G.N. Glavee, K.J. Klabunde, C.M. Sorensen, and G.C. Hadjipanayis, Langmuir, 10 (1994) 4726.

(92) G.N. Glavee, K.J. Klabunde, C.M. Sorensen, and G.C Hadjipanayis, Inorg. Chem., 34 (1995) 28. 
Table 1. Listing of the samples used in this study.

\begin{tabular}{|c|c|c|}
\hline NAME & DESCRIPTION & CHARACTERIZATION \\
\hline FeI & $\begin{array}{c}0.01 \mathrm{M} \mathrm{FeCl}{ }_{3}, 10 \text { wt. \% } \\
\text { DDAB/toluene }\end{array}$ & TEM \\
\hline FeII & $\begin{array}{c}0.01 \mathrm{M} \mathrm{Fe}\left(\mathrm{BF}_{4}\right)_{2}, 10 \text { wt. \% } \\
\mathrm{C}_{12} \mathrm{E}_{4} / \text { octane }\end{array}$ & Mossbauer, electron diffraction, XPS \\
\hline FeIII & FeI powder from methanol washing & $\begin{array}{c}\text { TEM, BET, XPS, NBM } \\
\text { hydrogenolysis }\end{array}$ \\
\hline FeIV & $\begin{array}{c}\text { FeIII re-dispersed with PVP and re- } \\
\text { dried }\end{array}$ & Mossbauer, XPS \\
\hline
\end{tabular}

Table 2. Mossbauer Parameters for FeII (Figure 3). $\mathrm{T}=-176^{\circ} \mathrm{C}$.

\begin{tabular}{|ccccc|}
\hline Assignment & $\begin{array}{c}\text { Isomer Shift } \\
(\mathrm{mm} / \mathrm{sec})\end{array}$ & $\begin{array}{c}\text { Quadrupole } \\
\text { Splitting } \\
(\mathrm{mm} / \mathrm{sec})\end{array}$ & $\begin{array}{c}\text { Magnetic } \\
\text { Hyperfine } \\
\text { Splitting (kOe) }\end{array}$ & \% Area \\
\hline $\begin{array}{c}\text { Doublet 1 } \\
(\mathrm{FeB})\end{array}$ & 0.470 & 0.69 & -- & 83.3 \\
$\begin{array}{c}\text { Doublet 2 } \\
\left(\mathrm{Fe}^{2+} \mathrm{BO}_{\mathrm{x}}\right)\end{array}$ & 1.300 & 3.08 & -- & 14.7 \\
Singlet $\left(\mathrm{Fe}^{0}\right)$ & -0.110 & -- & -- & 2.0 \\
\hline
\end{tabular}

${ }^{\text {arelative to iron }}$

Table 3. Mossbauer Parameters for FeIV (Figure 4). $\mathrm{T}=21^{\circ} \mathrm{C}$.

\begin{tabular}{|ccccc|}
\hline Assignment & $\begin{array}{c}\text { Isomer Shift } \\
(\mathrm{mm} / \mathrm{sec})\end{array}$ & $\begin{array}{c}\text { Quadrupole } \\
\text { Splitting } \\
(\mathrm{mm} / \mathrm{sec})\end{array}$ & $\begin{array}{c}\text { Magnetic } \\
\text { Hyperfine } \\
\text { Splitting (kOe) }\end{array}$ & \% Area \\
\hline $\begin{array}{c}\text { Doublet 1 } \\
(\mathrm{FeB})\end{array}$ & 0.450 & 0.27 & ---- & 60.4 \\
$\begin{array}{c}\text { Doublet 2 } \\
\left(\mathrm{Fe}^{2+} \mathrm{BO}_{\mathrm{x}}\right)\end{array}$ & 1.108 & 2.11 & ---- & 10.4 \\
Singlet $\left(\mathrm{Fe}^{0}\right)$ & -0.136 & -- & --- & 29.2 \\
\hline
\end{tabular}

arelative to iron 


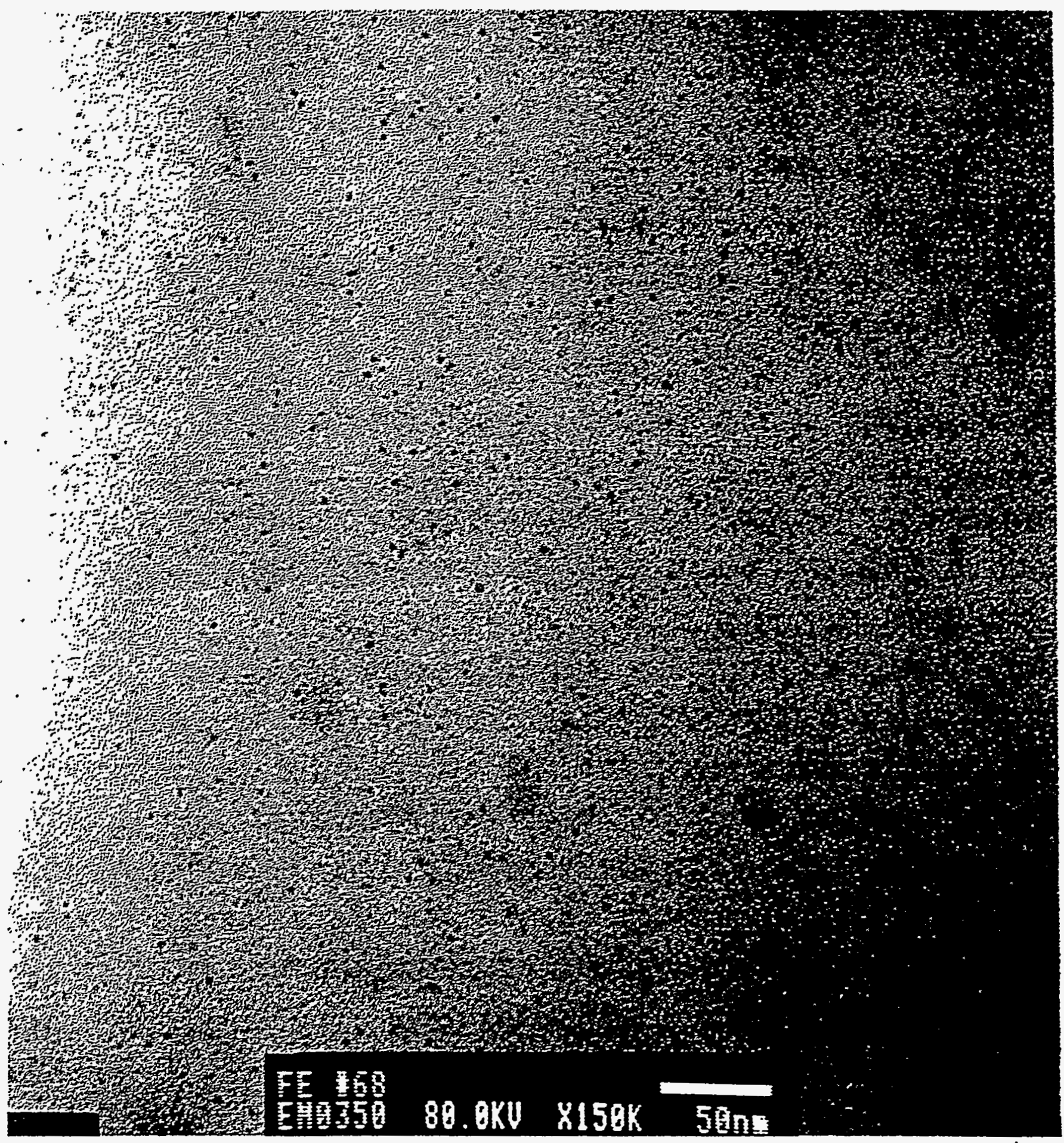

Figure 1. TEM of FeI particles. The number average diameter of the particles is $1.5+1-0.2$ nm. 


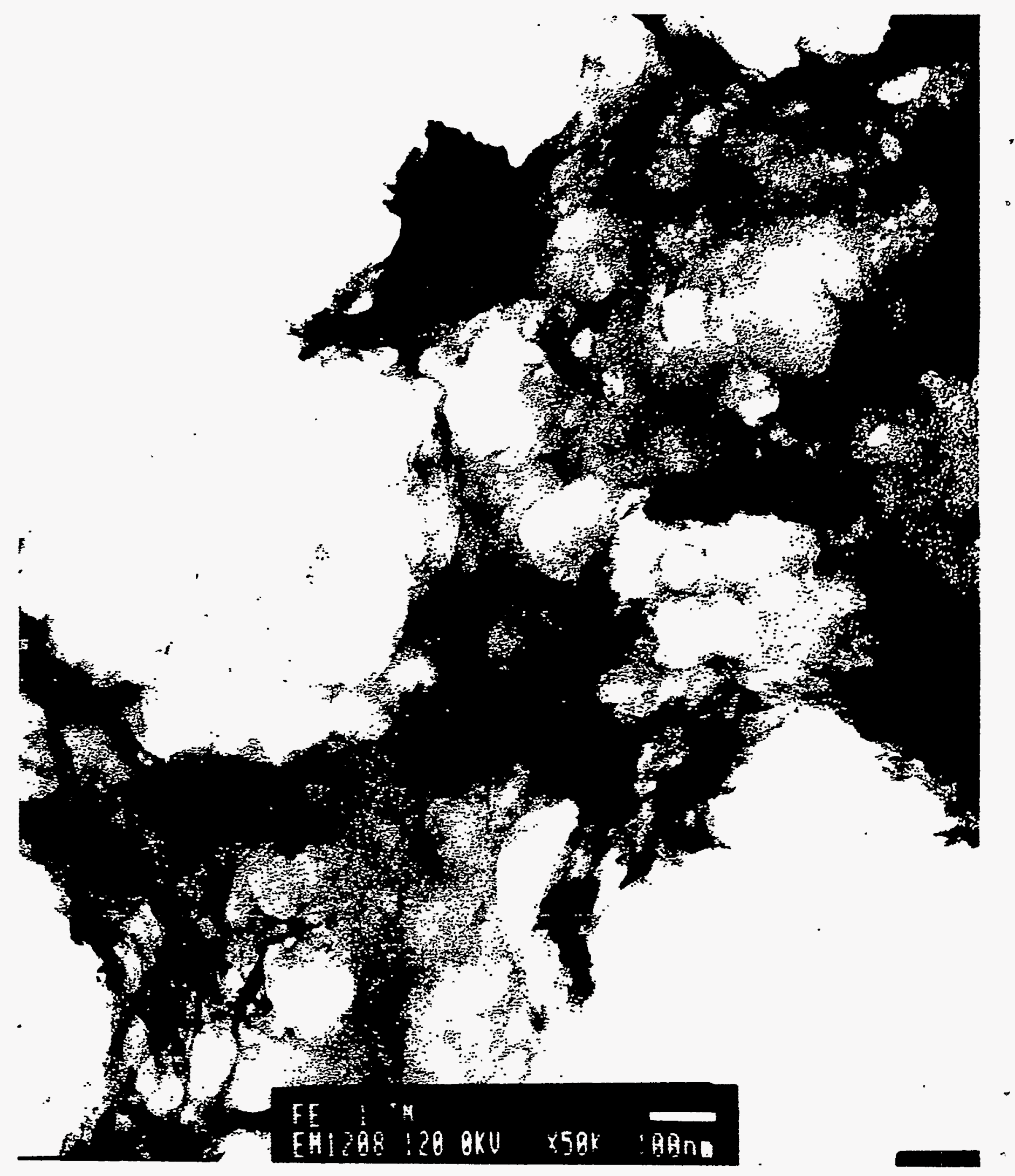

Figure 2. TEM of FeIII particles. After methanol washing the clusters aggregate and form particulate powders. 


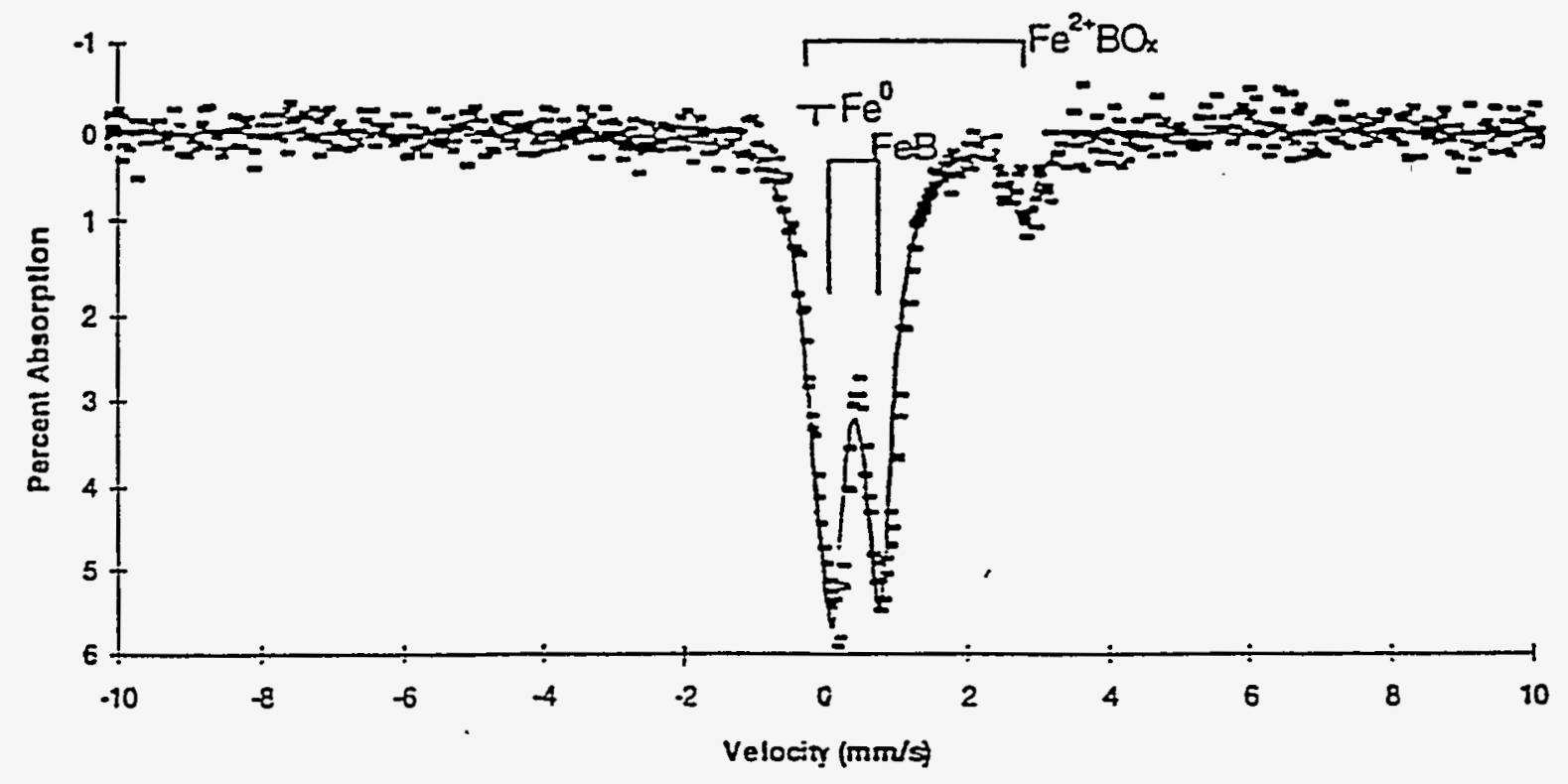

Figure 3. Mossbauer spectrum of Fell. $\mathrm{T}=-176^{\circ} \mathrm{C}$. Fit analysis listed in Table 2. 


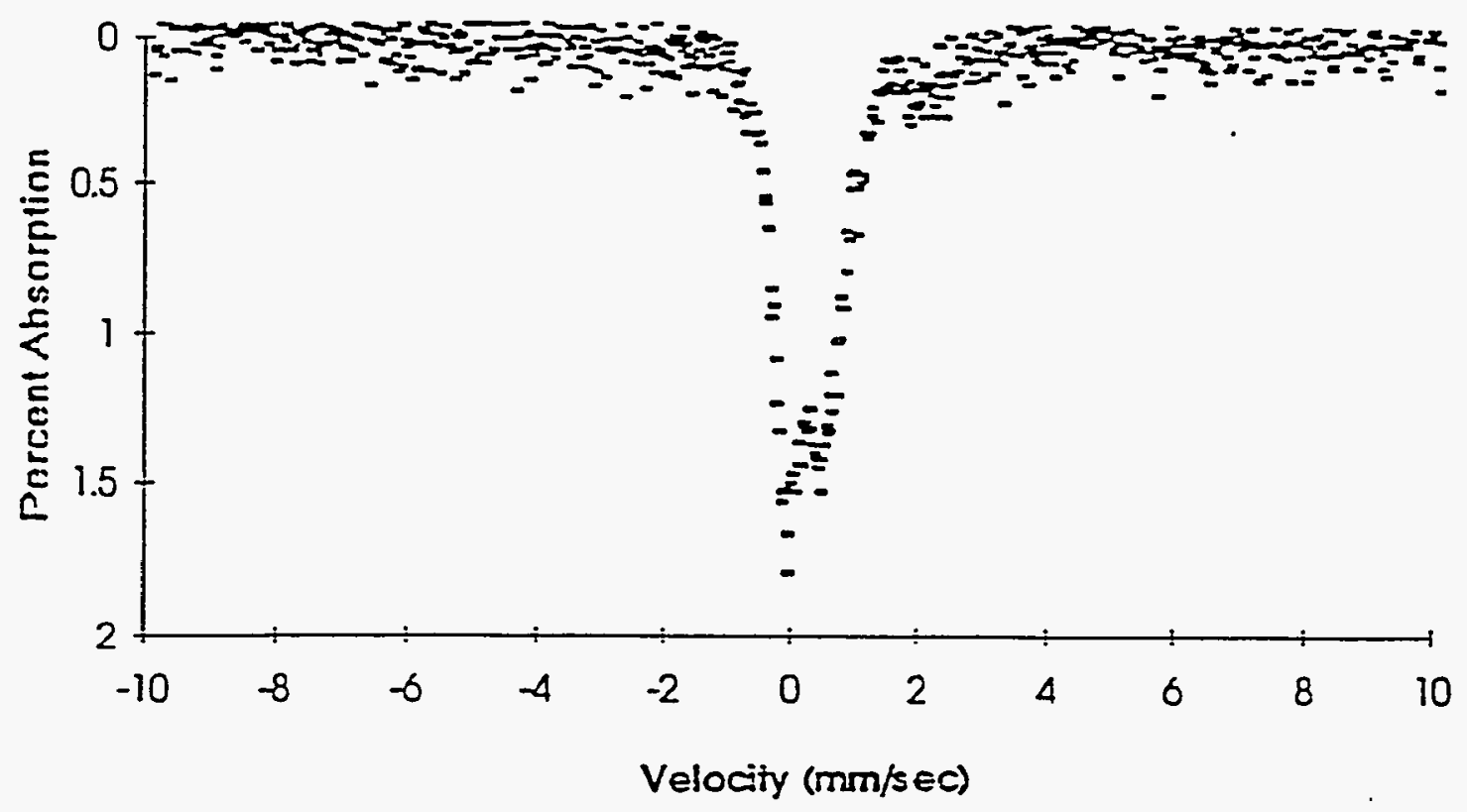

Figure 4. Mossbauer spectrum of FeIV. $\mathrm{T}=21^{\circ} \mathrm{C}$. Fit analysis listed in Table 3. 


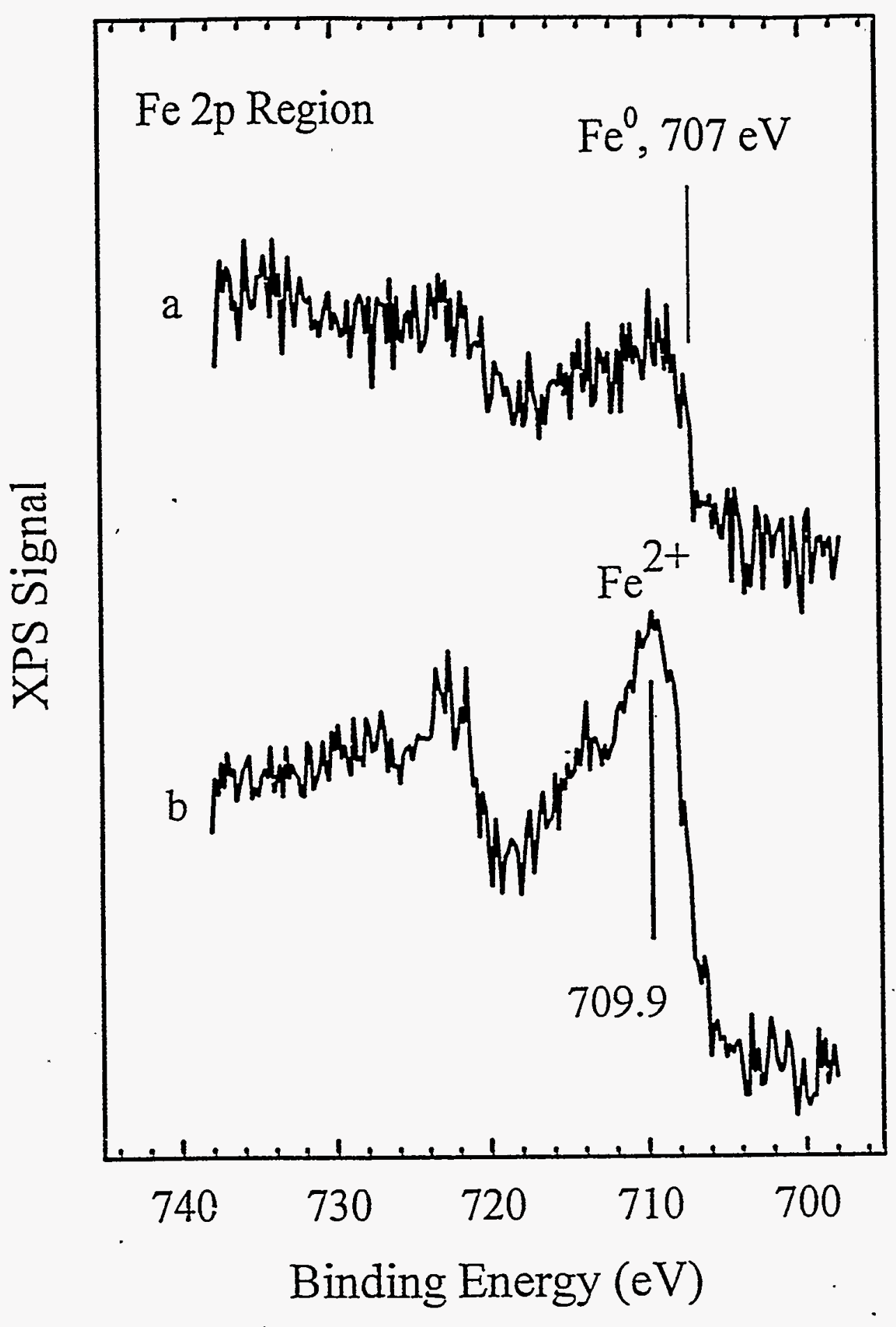

Figure 5a: XPS of the Fe 2p region of Fe particles embedded in PVP (FeIV). a) As prepared, b) heated to $250^{\circ} \mathrm{C}$ in vacuum. Note the single $\mathrm{Fe} 2 \mathrm{p}$ peak at $709.9 \mathrm{eV}$ with a shakeup feature at $-715 \mathrm{eV}$ characteristic of $\mathrm{Fe}^{2+}$. No appreciable intensity is observed at the position expected for zero valent iron. 


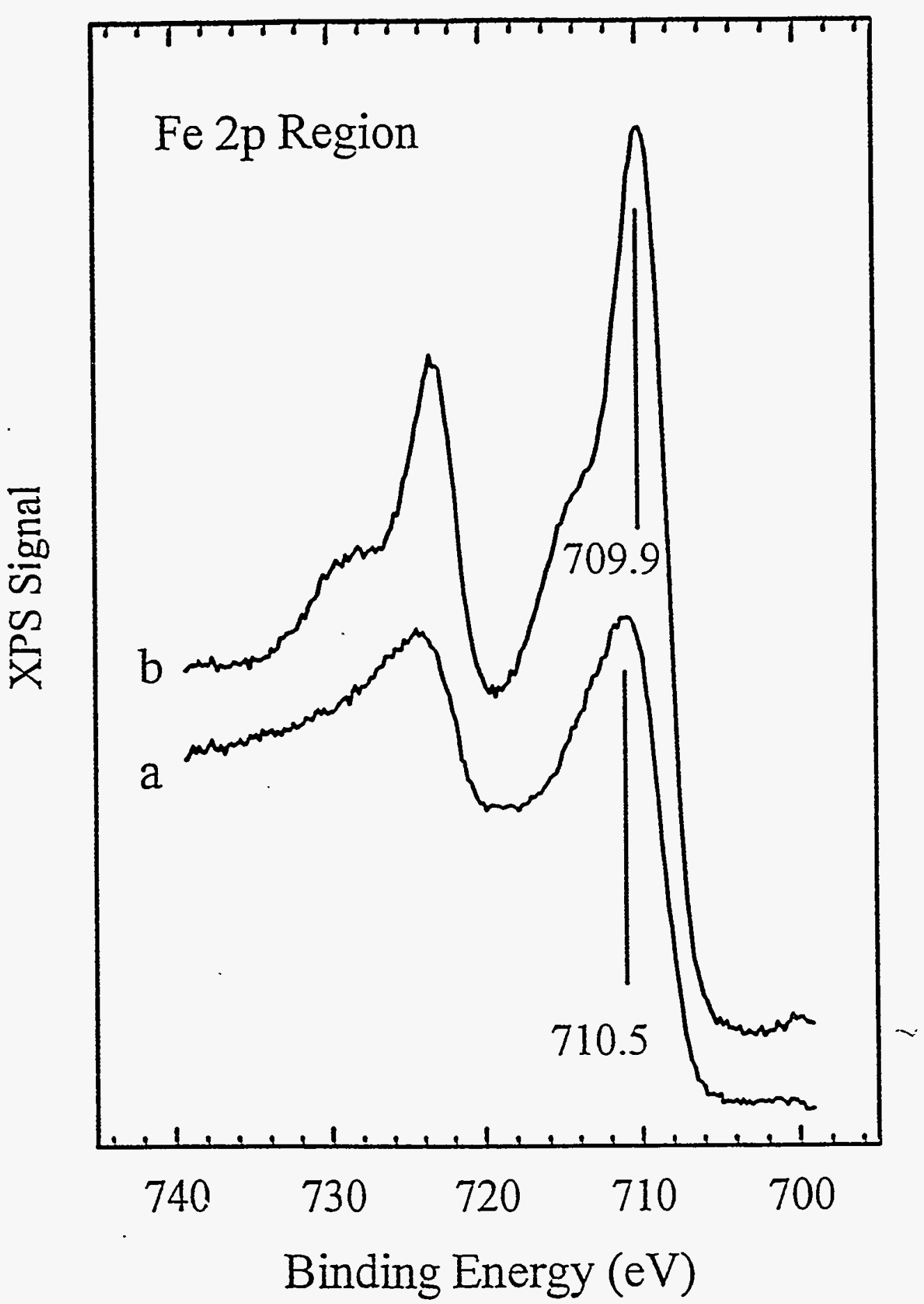

Figure 5b: XPS of the Fe 2p region of uncoated Fe particles (FeII). a) As prepared, b) heated to $300^{\circ} \mathrm{C}$ in vacuum. The absence of shakeup features in the as prepared spectrum is characteristic of a mixture of $\mathrm{Fe}^{2+}$ and $\mathrm{Fe}^{3+}$, while the shift of the $2 \mathrm{p} 3 / 2$ peak and the appearance of a shakeup feature after heating to $300^{\circ} \mathrm{C}$ indicate transformation of $\mathrm{Fe}^{3+}$ into $\mathrm{Fe}^{2+}$. No zero valent iron is observed at $707.0 \mathrm{eV}$. 


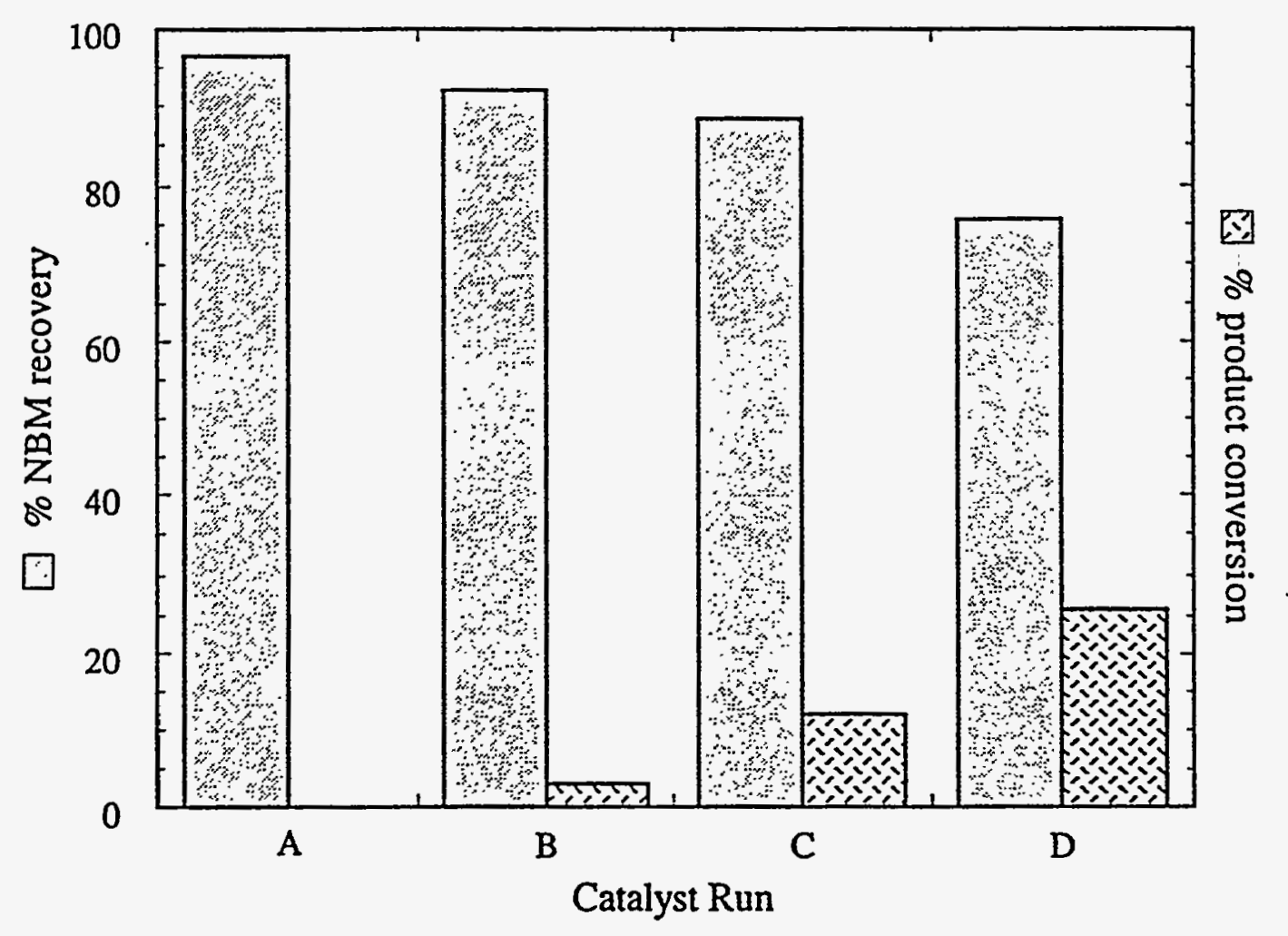

Figure 6a. NBM hydrogenolysis results for Fe clusters. The percentage of reactant recovered and percentage converted to product. $A=$ thermal average; $B=$ thermal with elemental $\mathrm{S}$ average; $\mathrm{C}=\mathrm{FeIII}(\sim 5 \%$ active metal basis $) ; \mathrm{D}=\mathrm{FeIII}$ with elemental $\mathrm{S}(\sim 5 \%$ active metal basis). 


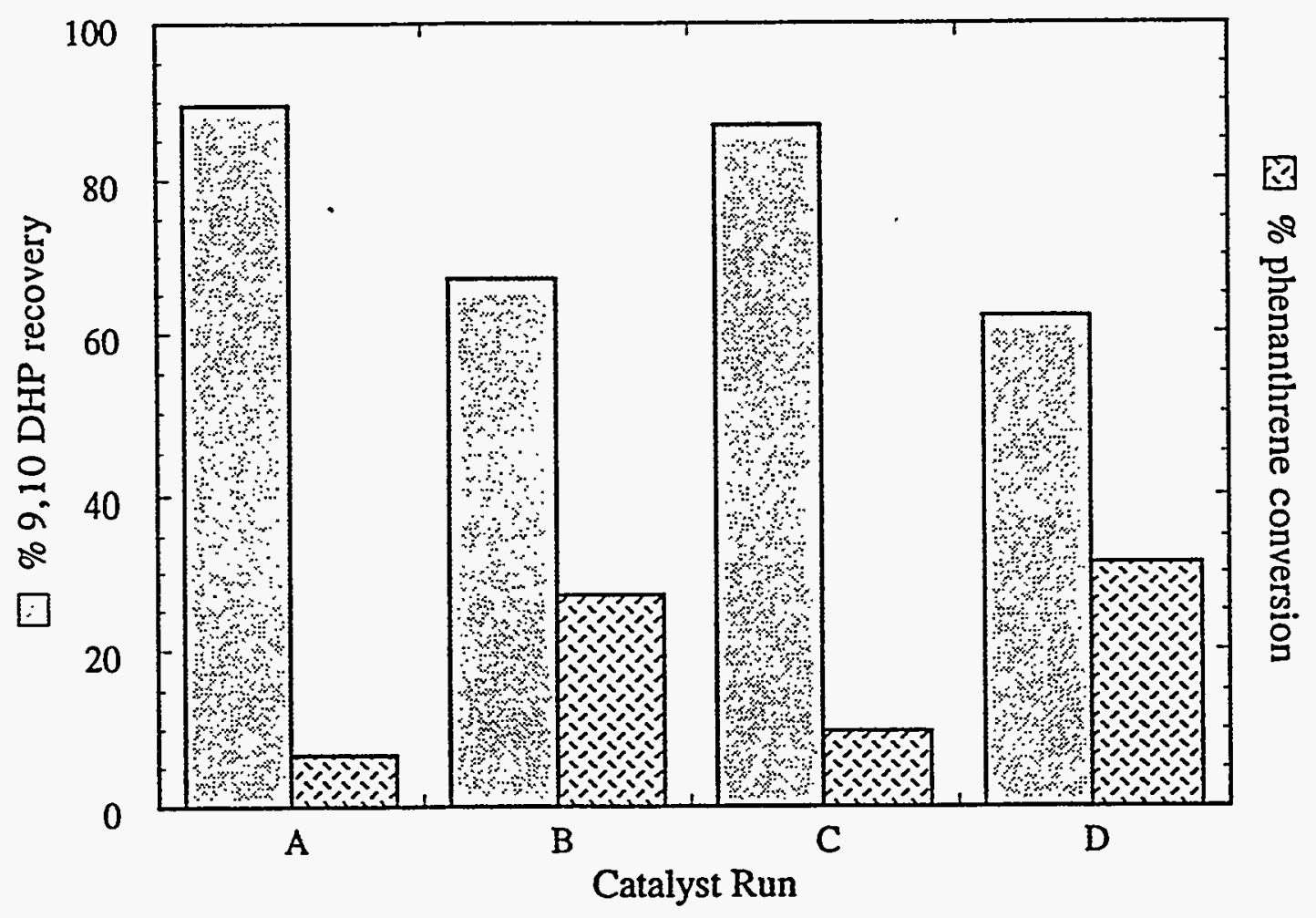

Figure $6 \mathrm{~b}$. NBM hydrogenolysis results for Fe clusters. The percentage of the hydrogen donating solvent $9,10 \mathrm{DHP}$ recovered and the percentage dehydrogenated to phenanthrene. $\mathrm{A}=$ thermal average; $\mathrm{B}=$ thermal with elemental $\mathrm{S}$ average; $\mathrm{C}=\mathrm{FeII}(\sim 5 \%$ active metal basis); $\mathrm{D}=$ Fell with elemental $\mathrm{S}$ ( $\sim 5 \%$ active metal basis). 


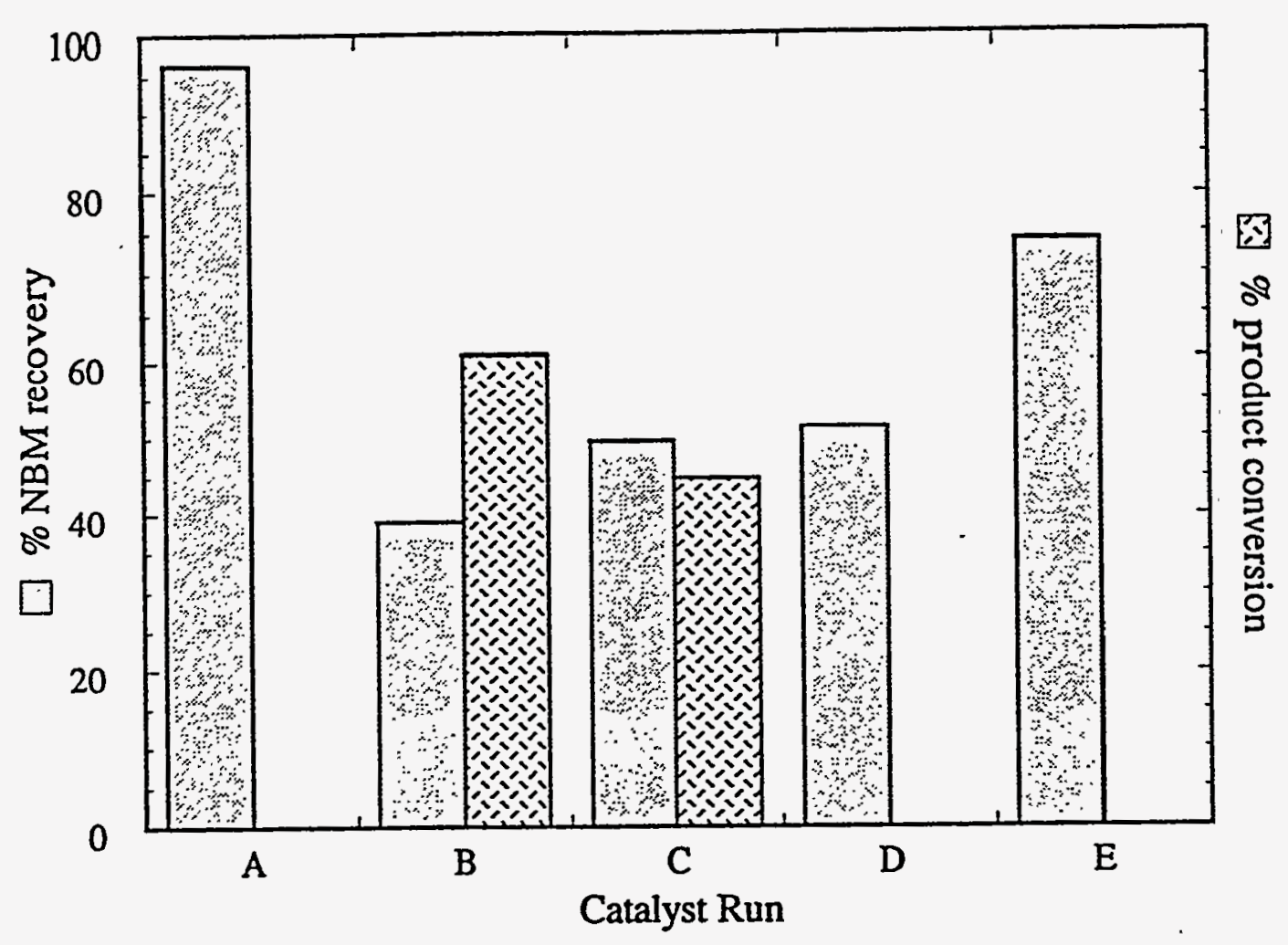

Figure 7a. NBM hydrogenolysis results for Shell 324 as a function of DDAB surfactant doping. The percentage of reactant recovered and percentage converted to product. $\mathrm{A}=$ thermal average; $B=$ Shell $324 ; C=$ Shell $324: D D A B=1: 4 ; D=$ Shell $324: D D A B=1: 20 ; E$ $=$ Shell $324: \mathrm{DDAB}=1: 50$. Active metal basis $\sim 5 \%$ for all catalyst runs. 


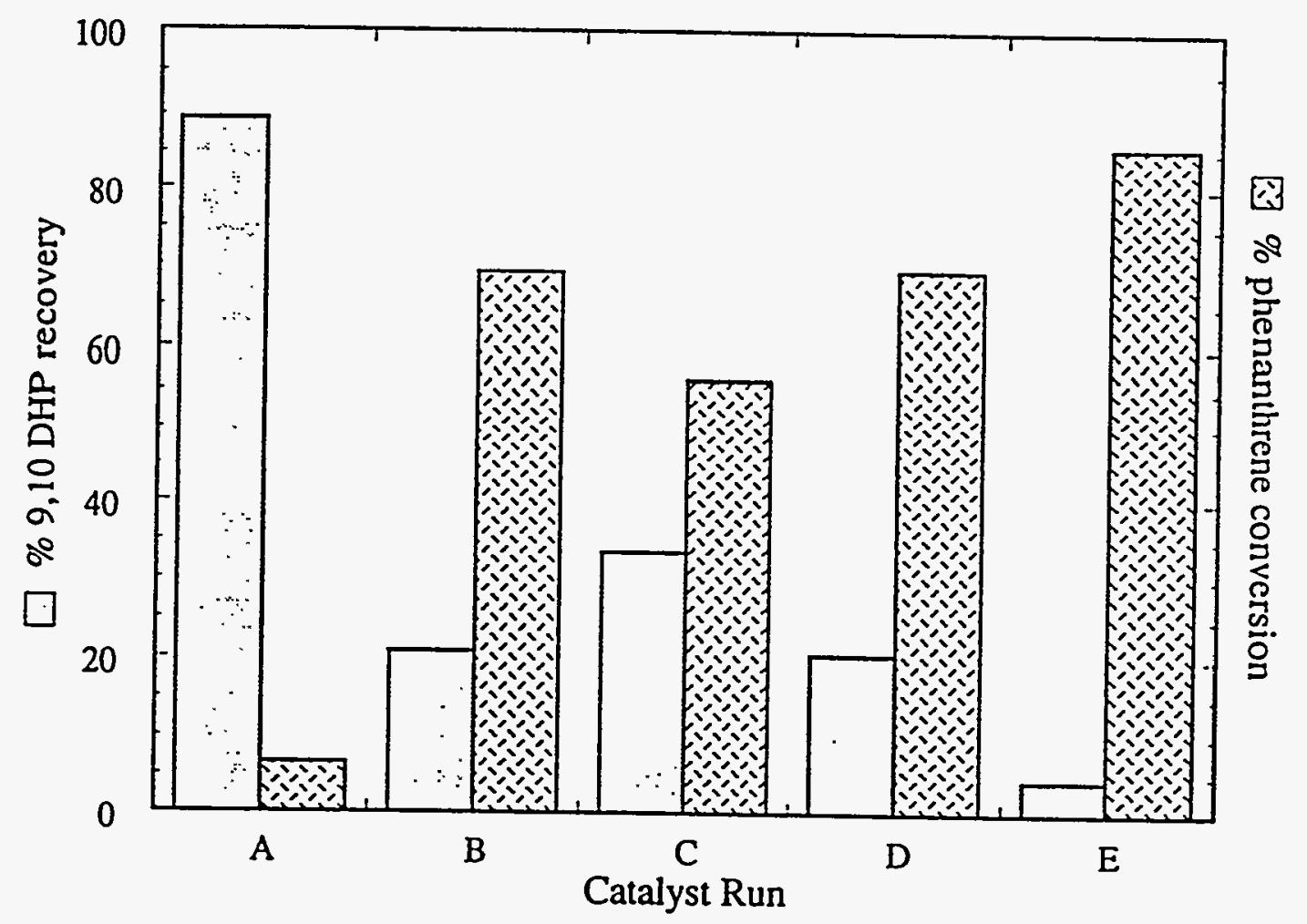

Figure 7b. NBM hydrogenolysis results for Shell 324 as a function of DDAB surfactant doping. The percentage of the hydrogen donating solvent 9, $10 \mathrm{DHP}$ recovered and the percentage dehydrogenated to phenanthrene. $A=$ thermal average; $B=$ Shell $324 ; C=$ Shell 324:DDAB =1:4; $\mathrm{D}=$ Shell 324:DDAB $=1: 20 ; \mathrm{E}=$ Shell 324:DDAB $=1: 50$. Active metal basis $\sim 5 \%$ for all catalyst runs. 


\title{
Chapter 3
}

\section{Preparation, Characterization, and Catalyst Testing of Highly Dispersed Rhodium Particles On Different Morphology Iron Oxide Supports.}

\begin{abstract}
The deposition of uniform $2-4 \mathrm{~nm}$ sized $\mathrm{Rh}$ particles on different shaped $\alpha$ $\mathrm{Fe}_{2} \mathrm{O}_{3}$ particles (rod shaped, spindle shaped, and cube shaped) and on $\beta-\mathrm{FeOOH}$ is achieved by the photochemical reduction of $\left[\left(\mathrm{C}_{2} \mathrm{H}_{4}\right)_{2} \mathrm{Rh}(\mathrm{OEt})\right]_{2} \cdot \mathrm{H}_{2} \mathrm{O}$ in the presence of the iron oxide. Deposition of the metal and synthesis of the support materials are independent, because the mild photochemical reduction does not effect the microstructure of the support. The Rh-coated iron oxide particles are characterized by electron microscopy, $\mathrm{x}$-ray diffraction, and thermal analysis. We have also studied the catalytic activity of the highly dispersed, high surface area Rh particles in a model hydrogenation reaction. Catalyst activity is dependent on the support composition ( $\beta$ $\mathrm{FeOOH}$ versus $\alpha-\mathrm{Fe}_{2} \mathrm{O}_{3}$ ) and independent on support morphology at the length scales studied $(-200 \mathrm{~nm})$. Finally, we have studied the effects of thermolysis on the dispersion of the rhodium particles, the microstructure of the support materials, and the consequent effects on the activity of the catalyst.
\end{abstract}

\section{Introduction}

Studies of nanometer sized metal particles are generating a great deal of interest due to their size-dependent properties. 1,2 These size-dependent properties exhibit themselves in both physical and chemical phenomena. Changes in physical phenomena such as electrical conductivity, optical absorption, and magnetism with size result from quantum-confinement effects. ${ }^{3-5}$ Changes is chemical phenomena such as catalytic reactivity and selectivity result from the high proportion of surface 
atoms having unsatisfied valences and low coordination numbers in ultra small particles. 6

We are interested in the preparation of nanometer sized $\mathrm{Rh}$ particles for heterogeneous (supported) catalysis applications. ${ }^{7-12}$ While there are a number of synthetic methods available to prepare heterogeneous catalysts $7-9,13-21$, photochemical reduction methods $22-24$ are attractive because they avoid high temperatures that change the support microstructure. 25 Photochemical preparation of metal on metal oxide materials from inorganic or metal-organic precursors is broadly divided into two strategies. The first strategy requires a photoactive support material, e.g. $\mathrm{TiO}_{2}$, to provide the electrons necessary to reduce the precursor complex. In this case, the metal is only deposited on the surface of the photoactive material and not on any insulating substrates. In the alternative strategy that we employ, the molecular precursor acts as the chromophore and metal may be deposited on the entire support surface regardless of composition.

In this work, we describe a method to deposit $\mathrm{Rh}$ on various supports by the photochemical reduction of a $\mathrm{Rh}(\mathrm{I})$ complex in the presence of the support. We study the synthesis, characterization, and activity of heterogeneous catalysts formed by the deposition of $\mathrm{Rh}$ on rod shaped, spindle shaped, and cube shaped $\alpha-\mathrm{Fe}_{2} \mathrm{O}_{3}$. This work is an extension of our previous work 12 in which we reported on the deposition of $2-4 \mathrm{~nm}$ sized $\mathrm{Rh}$ particles on rod-shaped $\beta-\mathrm{FeOOH}$ and spherical $\mathrm{SiO}_{2}$ particles. We further demonstrate the generality of the process and provide a comprehensive report on the catalytic activity of the materials. Because the deposition of the metal and the synthesis of the support are independent, we are able to study the deposition procedure and catalyst activity as a function of the support material. The catalysts are tested in the hydrogenation of pyrene, a model compound reaction representative of coal liquefaction processes. Finally, we investigate the thermal stability of the materials and the subsequent effect on catalytic activity.

\section{Experimental}

General Procedures and Instrumentation 
Hydrocarbon and ethereal solvents (toluene and tetrahydrofuran) were purchased from Aldrich Chemical Co. and pre-dried over sodium, distilled from sodium benzophenone ketyl, and stored over $4 \AA$ molecular sieves. Pyrene and anhydrous hexadecane was purchased from Aldrich Chemical Co. and used as delivered. Reagents $\mathrm{FeCl}_{3} \cdot 6 \mathrm{H}_{2} \mathrm{O}$ and $\mathrm{NaH}_{2} \mathrm{PO}_{4}$ were purchased from J.T. Baker Inc. The compound $\left[\left(\mathrm{C}_{2} \mathrm{H}_{4}\right)_{2} \mathrm{RhOEt}\right]_{2} \cdot \mathrm{H}_{2} \mathrm{O}$ was prepared as described previously. 9

Scanning electron microscopy (SEM) was completed with a Hitachi S-800, and transmission electron microscopy (TEM) was performed on a JOEL 2000-FX operating at $200 \mathrm{KeV}$. The reaction products were dispersed in acetone. A silicon wafer was dip-coated with the particles for SEM, and a carbon-coated copper grid was dip-coated for TEM. Electron diffraction revealed the presence or absence of crystallinity. X-ray powder diffraction was completed on a PVD-V Scintag X-ray diffractometer using a smear mount method to form a thin layer of sample onto a $3 x$ $3 \mathrm{~cm}^{2}$ piece of glass. Surface areas were measured the BET nitrogen adsorption method. Thermogravimetric analysis (TGA) was performed on a Perkin-Elmer TGA 7 instrument.

Rod-shaped, spindle-shaped, and cube-shaped particles of $\alpha-\mathrm{Fe}_{2} \mathrm{O}_{3}$ were prepared by modifications of literature procedures as described below. $26-28$ The deposition of $\mathrm{Rh}$ on these materials is also described below. Rod shaped $\beta-F e O O H$ particles and rod-shaped $\beta-\mathrm{FeOOH}$ with $2.4 \mathrm{wt} . \%$ and $11.4 \mathrm{wt} \% \mathrm{Rh}$ were prepared as described previously. 12 All manipulations were carried out under a dinitrogen atmosphere using standard Schlenk techñiques. 29

\section{Syntheses}

1. Rod, Spindle, and Cube-Shaped $\alpha-\mathrm{Fe}_{2} \mathrm{O}_{3}$

Rod-Shaped $\alpha-\mathrm{Fe}_{2} \mathrm{O}_{3}:$ Rod-shaped $\alpha-\mathrm{Fe}_{2} \mathrm{O}_{3}$ is produced from the thermal decomposition of rod-shaped $\beta$-FeOOH. Rod-shaped $\beta$-FeOOH, $0.304 \mathrm{~g}(3.42$ mmole), was heated at $400^{\circ} \mathrm{C}$ in vacuo for 2 hour, and $0.244 \mathrm{~g}(1.53 \mathrm{mmole})$ of the red-brown product was obtained. The corresponding yield of the process is $89 \%$ based on $\mathrm{Fe}_{2} \mathrm{O}_{3}$. 
Spindle-Shaped $\alpha-\mathrm{Fe}_{2} \mathrm{O}_{3}: 2.703 \mathrm{~g}(10 \mathrm{mmole})$ of $\mathrm{FeCl}_{3} \cdot 6 \mathrm{H}_{2} \mathrm{O}$ and $0.138 \mathrm{~g}$ (1.0 mmole) of $\mathrm{NaH}_{2} \mathrm{PO}_{4}$ were dissolved in about $100 \mathrm{~cm}^{3}$ of distilled water. The solution was filtered and diluted to $500 \mathrm{~cm}^{3}$, transferred into a $1000 \mathrm{~cm}^{3}$-Erlenmeyer flask, and sealed with a screw cap. The Erlenmeyer flask was then placed into a preheated oven and kept at $100^{\circ} \mathrm{C}$ for 48.5 hours. The color of the solution changed from yellow to dark-brown. After cooling, the solid was separated by centrifugation, washed six times with distilled water, three times with acetone, and dried at ambient temperature to give $0.674 \mathrm{~g}$ of final product. The corresponding yield of the process is $84 \%$ based on $\mathrm{Fe}_{2} \mathrm{O}_{3}$.

Cube-Shaped $\alpha-\mathrm{Fe}_{2} \mathrm{O}_{3}: 2.4336 \mathrm{~g}$ ( 9 mmole) of $\mathrm{FeCl}_{3}{ }^{\bullet} 6 \mathrm{H}_{2} \mathrm{O}$ was dissolved in $100 \mathrm{~cm}^{3}$ of distilled water with $5 \mathrm{~cm}^{3}$ of $0.1 \mathrm{M} \mathrm{HCl}$. The solution was filtered and diluted to $500 \mathrm{~cm}^{3}$, transferred to a $1000 \mathrm{~cm}^{3}$-Erlenmeyer flask, and sealed with a screw cap. The Erlenmeyer flask was then placed into a preheated oven and kept at $100^{\circ} \mathrm{C}$ for 24 hours. The color of the solution changed from yellow to brown. After cooling, the solid was separated by centrifugation, washed six times with distilled water, three times with acetone, and dried at ambient temperature to give $0.287 \mathrm{~g}$ of final product. The corresponding yield of the process is $40 \%$ based on $\mathrm{Fe}_{2} \mathrm{O}_{3}$.

2. Deposition of Rh Particles by Photochemical Reduction

$\mathrm{Rh}$ particles were dispersed on each of the three morphologies of $\alpha-\mathrm{Fe}_{2} \mathrm{O}_{3}$ through photoreduction of $\left[\left(\mathrm{C}_{2} \mathrm{H}_{4}\right)_{2} \mathrm{RhOEt}\right]_{2} \cdot \mathrm{H}_{2} \mathrm{O}$ in the presence of the $\alpha-\mathrm{Fe}_{2} \mathrm{O}_{3}$ samples. Three Schlenk flasks were charged separately under $\mathrm{N}_{2}$ with $7.5 \mathrm{mg}(0.018$ mmole) of $\left[\left(\mathrm{C}_{2} \mathrm{H}_{4}\right)_{2} \mathrm{RhOEt}\right] 2^{\cdot} \mathrm{H}_{2} \mathrm{O}$ and $150 \mathrm{mg}$ ( $\left.0.939 \mathrm{mmole}\right)$ of rod-shaped $\alpha$ $\mathrm{Fe}_{2} \mathrm{O}_{3}, 10 \mathrm{mg}(0.023 \mathrm{mmole})$ of $\left[\left(\mathrm{C}_{2} \mathrm{H}_{4}\right)_{2} \mathrm{RhOEt}\right]_{2} \cdot \mathrm{H}_{2} \mathrm{O}$ and $200 \mathrm{mg}(1.25 \mathrm{mmole})$ of spindle-shaped $\alpha-\mathrm{Fe}_{2} \mathrm{O}_{3}$, and $8 \mathrm{mg}(0.019 \mathrm{mmole})$ of $\left[\left(\mathrm{C}_{2} \mathrm{H}_{4}\right)_{2} \mathrm{RhOEt}\right]_{2} \cdot \mathrm{H}_{2} \mathrm{O}$ and $154 \mathrm{mg}(0.964 \mathrm{mmole})$ of cube-shaped $\alpha-\mathrm{Fe}_{2} \mathrm{O}_{3}$. A stir bar and $15 \mathrm{~cm}^{3}$ of THF was added to each flask. The suspensions were dispersed by indirect sonication for one minute and stirred at room temperature in the dark for about 30 minutes. The mixtures were then irradiated with a mercury lamp for 2 hours. The products were separated by filtration, washed with acetone, and dried in air. The filtrates of $\mathrm{Rh}$ on 
rod-shaped and spindle-shaped $\alpha-\mathrm{Fe}_{2} \mathrm{O}_{3}$ are colorless while the filtrate of $\mathrm{Rh}$ on cube-shaped $\alpha-\mathrm{Fe}_{2} \mathrm{O}_{3}$ shows a light yellow color. Assuming quantitative reaction of the $\mathrm{Rh}(\mathrm{I})$ complex, the metal loading on the supports is $2.4 \mathrm{wt}$. \%.

\section{Catalytic Pyrene Hydrogenation}

Hydrogenation of pyrene was carried out in a Berghof Teflon lined microbatch reactor. The reactor was charged with $100 \mathrm{mg}$ pyrene and $1000 \mathrm{mg}$ of hexadecane solvent, and the reactions were run at $100+/-3^{\circ} \mathrm{C}, 100$ psig cold $\mathrm{H}_{2}$, for $30 \mathrm{~min}$. Two catalyst loadings were tested: $0.5 \mathrm{wt}$. \% and $2.3 \mathrm{wt} . \%$ catalyst on a Rh to pyrene basis. Hydrogenation products for this reaction include isodecahydropyrene, decahydropyrene, tetrahydropyrene, iso-hexahydropyrene, hexahydropyrene, and dihydropyrene. Products were worked up in toluene and product analysis was completed by gas chromatography. Activity is reported in turnover numbers (TON) defined by: [ (number of moles of pyrene reacted)/(number of moles of $\mathrm{Rh} \cdot$ time) ].

\section{Results and Discussion}

Characterization of 2-4 $\mathrm{nm}$ Rh Particles on Rod-Shaped, Spindle Shaped, and CubeShaped $\alpha-\mathrm{Fe}_{2} \mathrm{O}_{3}$

The synthesis described in the previous section produced rod-shaped, spindleshaped, and cube-shaped $\alpha-\mathrm{Fe}_{2} \mathrm{O}_{3}$. X-ray powder diffraction confirmed the presence of crystalline $\alpha-\mathrm{Fe}_{2} \mathrm{O}_{3}$ in the rod-shaped material, and X-ray powder diffraction and electron diffraction confirmed the presence of crystalline $\alpha-\mathrm{Fe}_{2} \mathrm{O}_{3}$ in the spindle and cube-shaped material. The lattice fringe images observed by high resolution TEM verify that individual particles of spindle and cube-shaped $\alpha-\mathrm{Fe}_{2} \mathrm{O}_{3}$ are single crystals. The spindle-shaped particles are approximately $325 \mathrm{~nm}$ long and $125 \mathrm{~nm}$ in diameter, and the average cube-shaped particle size is $150 \mathrm{~nm}$. BET surface areas of the rod, spindle, and cube shaped $\alpha-\mathrm{Fe}_{2} \mathrm{O}_{3}$ powders are $8.6 \mathrm{~m}^{2} / \mathrm{g}, 11.3 \mathrm{~m} / \mathrm{g}$, and 7.8 $\mathrm{m}^{2} / \mathrm{g}$, respectively. 
After deposition of $\mathrm{Rh}$ by photochemical reduction, the red-brown rod-shaped powders turned brown, and the brown spindle-shaped powders and red-brown cubeshaped powders darkened. In all cases, it is seen that $2-4 \mathrm{~nm}$ particles of $\mathrm{Rh}$ are uniformly dispersed on the support materials. As in our previous work, we have shown that the photochemical deposition procedure is independent of support material $\left(\mathrm{Fe}_{2} \mathrm{O}_{3}, \mathrm{FeOOH}\right.$, and $\left.\mathrm{SiO}_{2}\right)$. Quantitative deposition of the $\mathrm{Rh}$ on the support resulted as no unreacted $\left[\left(\mathrm{C}_{2} \mathrm{H}_{4}\right) 2 \mathrm{Rh}(\mathrm{OEt})\right]_{2} \cdot \mathrm{H}_{2} \mathrm{O}$ exists in solution after reaction as determined by ${ }_{1}$ HNMR spectroscopy of the material contained in the supernatant. Energy dispersive spectroscopy confirms the presence of $\mathrm{Rh}$ in all samples. No Rh particles were detected in the dispersed solution (even after sonication) indicating the particles adhere strongly to the support surface.

Thermolysis of Rod-, Spindle-, and Cube-Shaped $\alpha-\mathrm{Fe}_{2} \mathrm{O}_{3}$, and of $\beta$-FeOOH and $\mathrm{Rh}$ coated $\beta-F e O O H$

In order to determine the effect of heat treatment in these materials, model experiments were carried out wherein the $\mathrm{Fe}_{2} \mathrm{O}_{3}$ and the $\beta-\mathrm{FeOOH}$ supports were heated and characterized by crystallography and microscopy. The spindle and cubeshaped $\alpha-\mathrm{Fe}_{2} \mathrm{O}_{3}$ particles exhibited a total weight loss of $3.5 \%$ and $1.0 \%$, respectively, on heating in static air up to $800^{\circ} \mathrm{C}$. The weight losses are probably due to the dehydration of surface hydroxyl groups. Heating these samples up to $800^{\circ} \mathrm{C}$ in a 7\% $\mathrm{H}_{2}$ in $\mathrm{N}_{2}$ atmosphere resulted in weight losses of $32.3 \%$ and $31.0 \%$ for spindleand cube-shaped powders, respectively. The weight losses are consistent with the formation of Fe.

We have shown that rod-shaped $\alpha-\mathrm{Fe}_{2} \mathrm{O}_{3}$ is produced by the thermal decomposition of rod-shaped $\beta-\mathrm{FeOOH}$ at $400^{\circ} \mathrm{C}$ in vacuo. It is interesting to note that the synthesis is strongly dependent on temperature and atmosphere. At $300^{\circ} \mathrm{C}$, an amorphous iron oxide is produced that gives no diffraction pattern. The amorphous material has a BET surface area of $54.2 \mathrm{~m}^{2} / \mathrm{gm}$, more than twice that of the original rod-shaped $\beta-F e O O H$ powder. At $400^{\circ} \mathrm{C}$, in air rather than in vacuo, an amorphous iron oxide is once again the product. The rod-shaped morphology remains, but no 
diffraction pattern is detected. TEM indicates the creation of an approximately $10 \mathrm{~nm}$ channel structure that does not exist in the $\beta-\mathrm{FeOOH}$. An increase in microporosity of the amorphous material could account for the increase in the BET surface area. At present we speculate that the amorphous structure is derived from the removal of water and interstitial ions $\left(\mathrm{Cl}^{-}\right)$from the $\beta-\mathrm{FeOOH}$ structure ${ }^{30}$, and that thermolysis under air rather than vacuum allows the gross morphology to be retained while the porous internal microstructure can develop. Further investigation is clearly warranted.

On heating $\mathrm{Rh}$ loaded $\beta-\mathrm{FeOOH}$ particles (synthesis described previously ${ }^{12}$ ) to $400^{\circ} \mathrm{C}$ in air, the transition to the same amorphous iron oxide material occurs. $\mathrm{X}$ ray diffraction shows only the pattern consistent with fcc-Rh. No agglomeration of the $\mathrm{Rh}$ particles is detected.

\section{Catalytic Hydrogenation of Pyrene}

Polynuclear aromatic hydrocarbons (PAHs) are vital in the liquefaction of coal to viable fuels, because they assist hydrogen transfer between a hydrogen source and free radical coal fragments formed in the liquefaction process. 31 Hydrogenation of the PAH pyrene models the kinetics and thermodynamics of the hydrogenation of PAHs in liquefaction reactions ${ }^{32}$ and so the reaction is studied. Hydrogenation of pyrene to form 4,5-dihydropyrene is modeled by pseudo first order reversible kinetics, and the reaction proceeds at moderate conditions $\left(\mathrm{T}=100^{\circ} \mathrm{C}, \mathrm{P}=100 \mathrm{psi}\right.$ $\mathrm{H}_{2}$ ) in the presence of a catalyst. Catalysts studied in the past include $\mathrm{Ni}^{33}, \mathrm{NiMo}^{34}$, $\mathrm{Pd}^{35}, \mathrm{Fe}^{36}, \mathrm{MoS}_{2}{ }^{37}$, and $\mathrm{Rh}^{8}$. We have studied the heterogeneous $\mathrm{Rh}$ catalysts synthesized here as a function of the support material, thermal treatment, and metal loading.

Background runs on the three types of $\alpha-\mathrm{Fe}_{2} \mathrm{O}_{3}, \beta-\mathrm{FeOOH}$, and $\mathrm{SiO}_{2}$ show no catalytic activity; no products are detected giving a TON of zero. The materials become catalytic in the presence of $\mathrm{Rh}$, and activity is a function of the active metal concentration (Figure 1). Support composition dependent catalytic activity is clearly noted (Figure 2). The activity of the $\mathrm{Rh}$ on $\beta$ - $\mathrm{FeOOH}$ catalyst is twice that of 
previously studied Rh catalysts ${ }^{8}$ and four times greater than Pd catalysts ${ }^{35}$ studied at the same conditions. Activity of the heterogeneous Rh catalysts decreases by factors of two as a function of support material for $\alpha-\mathrm{Fe}_{2} \mathrm{O}_{3}$ and then $\mathrm{SiO}_{2}$. The activity of $\mathrm{Rh}$ on rod-, spindle-, and cube-shaped $\alpha-\mathrm{Fe}_{2} \mathrm{O}_{3}$ catalysts is relatively constant (Figure 2) indicating that activity is independent on the morphology of the support material.

The effect of the thermolysis of $\mathrm{Rh}$ on $\beta-\mathrm{FeOOH}$ is minimal (Figure 3). Catalytic activity decreases slightly in the temperature treated sample. In the temperature treated sample, the dispersion of the metal and the morphology of the support remain constant, the crystalline $\beta$ - $\mathrm{FeOOH}$ becomes amorphous, and a channel structure in the amorphous material develops. Decrease in the catalytic activity must result from the chemical change in the support that produces the amorphous structure.

Finally, catalytic activity is determined as a function of the metal loading on the support (Figure 4). At constant active metal concentration with respect to the reactant pyrene, catalytic activity drops substantially in the material highly loaded with $\mathrm{Rh}$. Lower activity is consistent with lower dispersion and larger particle sizes observed in the highly loaded $\mathrm{Rh}$ on $\beta$-FeOOH material.

\section{Conclusions:}

We have prepared 2-4 $\mathrm{nm}$ sized $\mathrm{Rh}$ particles dispersed on a variety of supports by the photochemical reduction of $\left[\left(\mathrm{C}_{2} \mathrm{H}_{4}\right)_{2} \mathrm{Rh}(\mathrm{OEt})\right]_{2} \cdot \mathrm{H}_{2} \mathrm{O}$ in the presence of the supports. Rh particles were dispersed on support materials of different morphology and composition (rod-, spindle-, cube-shaped $\alpha-\mathrm{Fe}_{2} \mathrm{O}_{3}, \beta-\mathrm{FeOOH}$, and $\mathrm{SiO}_{2}$ ) indicating the procedure is general. The mild photochemical reduction does not effect the microstructure of the support. Quantitative deposition of the $\mathrm{Rh}$ on the support resulted as no unreacted $\left[\left(\mathrm{C}_{2} \mathrm{H}_{4}\right)_{2} \mathrm{Rh}(\mathrm{OEt})\right]_{2} \cdot \mathrm{H}_{2} \mathrm{O}$ or $\mathrm{Rh}$ particles exist in solution after reaction. Catalytic hydrogenation testing of the materials indicate that activity is a function of the composition of the support but not the morphology of the support at the length scales studied. Activity is also a function of particle size as effected by an increase in the metal loading of the material. Thermolysis of the $\beta$-FeOOH supported 
material shows that the dispersion of the metal and the morphology of the support remain constant, the crystalline $\beta-\mathrm{FeOOH}$ becomes amorphous, and a channel structure in the amorphous material develops. As a result, catalytic activity of the heat treated $\mathrm{Rh}$ on $\beta$ - $\mathrm{FeOOH}$ decreases.

Acknowledgments:

This work was funded by the Department of Energy, contract number DEAC04-76DP00789. The authors thank the NSF Chemical Instrumentation program for the purchase of a low field NMR spectrometer, Dr. Lu-Min Wang for assistance in obtaining some of the TEM data on a JEOL 2000-FX in the Department of Earth and Planetary Sciences, UNM, Dongshui and Leo Archer for X-ray powder diffraction data and Ranjan Ghosal for surface area measurements. We also thank ONR for analytical facilities and Dr. A. Sylwester for helpful discussions. 


\section{References}

(1) P. Marquardt, G. Nimtz and B. Muhlschlegel Solid State Communications 1988, $65,539$.

(2) See for example,Science 1992, 255, 1049.

(3) M. L. Steigerwald and L. E. Brus Acc. Chem. Res. 1990, 23, 183.

(4) Y. Yang and N. Herron J. Phys. Chem. 1991, 95, 525.

(5) P. O'Brien, in Inorganic Materials, (Edited by D. W. Bruce and D. O'Hare), p. 491. John Wiley \& Sons Ltd., (1992).

(6) M. Che and C. O. Bennett, in Advances in Catalysis, (Edited by D. D. Eley, H. Pines and P. B. Weisz), Vol. 36, p. 55. Academic, New York (1989).

(7) Z. Duan, M. J. Hampden-Smith, A. Datye, P. J. Nigrey, C. Quintana and A. P. Sylwester, in Chemical Processes in Inorganic Materials: Metal and Semiconducter Clusters and Colloids, (Edited by J. S. Bradley, P. D. Persans, G. Schmid and R. R. Chianelli), Vol. 272, p. 109. Mat. Res. Soc. Symp. Proc., Pittsburgh (1992).

(8) Z. Duan, M. J. Hampden-Smith, D. A., P. Nigrey, C. Quintana and A. P. Sylwester J. Catal. 1993, 139, 504.

(9) Z. Duan, M. J. Hampden-Smith and A. P. Sylwester J. Organomet. Chem. 1993, 449, 173.

(10) Z. Duan, M. J. Hampden-Smith and A. P. Sylwester Chem. Mater. 1992, 4, 1146.

(11) Z. Duan and M. J. Hampden-Smith published (1993).

(12) Z. Duan and M. J. Hampden-Smith Chem. Mater. 1993, 5, 994.

(13) S. C. Davis and K. J. Klabunde Chem. Rev. 1982, 82, 153.

(14) G. Brauer Handbook of preparative Inorganic Chemistry. 2nd ed., Vol. 2. Academic, New York, (1965).

(15) R. D. Rieke Science 1989, 246, 1260.

(16) H. Bönnemann, W. Brijoux, R. Brinkmann, E. Dinjus, T. Joussen and B. Korall Angew. Chem. Int. Ed. Engl. 1991, 30, 1312.

(17) H. Bönnemann, W. Brijoux and T. Joussen Angew. Chem. Int. Ed. Engl. 1990, 29, 273.

(18) J. S. Bradley, J. M. Millar, E. W. Hill and S. Behal J. Catal. 1991, 129, 530.

(19) J. S. Bradley, J. M. Millar, E. W. Hill and M. Melchior J. Chem. Soc., Chem. Comm. 1990, 705,

(20) J. S. Bradley, J. M. Millar and E. W. Hill J. Am. Chem. Soc. 1991, 113, 4016.

(21) H. Glassl, R. Hayek and R. Kramer J. Catal. 1981, 68, 397.

(22) J. M. Herrmann and M. J.L. J. Catal. 1990, 121, 340.

(23) J. M. Herrmann, J. Disdier, P. Pichat, A. Fernandez, A. Gonzalez-Elipe, G. Munuera and C. Leclercq J. Catal. 1991, 132, 490, and references therein.

(24) A. Fernandez, A. R. Gonzalez-Elipe, C. Real, A. Caballero and G. Munuera Langmuir 1993, 9, 121.

(25) C. J. Brinker and G. W. Scherer Sol-Gel Science, The Physics and Chemistry of Sol-Gel Processing. Academic Press, (1990).

(26) E. Matijevic and P. Scheiner J. Coll. Interface Sci. 1978, 63, 509.

(27) M. Ozaki, S. Kratohvil and E. Matijevic J. Colloid Interface Sci. 1984, 102, 146. 
(28) R. Partch, S. G. Gangolli, E. Matijevic, W. Cai and S. Arajs J. Colloid Interface Sci. 1991, 144, 27.

(29) D. F. Shriver and M. A. Drezden The Manipulation of Air-Sensitive Compounds. 2nd ed., pp 78. Wiley- Interscience, New York, (1986).

(30) A. F. Wells, in Structural Inorganic Chemistry, 5th ed.(1986).

(31) D.D. Whitehurst, T.O. Mitchell and M. Farcasiu, in Coal Liquefaction, Academic Press, New York, (1980).

(32) H.P. Stephens and R.J. Kottenstette J. Am. Chem. Soc., Div. Fuel Chem. 1985, $30(2), 345$.

(33) E.S. Olson and R.K. Sharma J. Am. Chem. Soc., Div. Fuel Chem. 1994, 39(3), 706.

(34) T.J. Gardner, L.I. McLaughlin, S.E. Lott, and J.B. Oelfke J. Am. Chem Soc., Div. Fuel Chem. 1994, 39(4), 1078.

(35) R.G. Dosch, F.V. Stohl, and J.T. Richardson ACS Symp. Ser. 1990, 437, 279.

(36) Y. Tang and C.W. Curtis Energy Fuels 1994, 8(1), 63.

(37) P.S. Ting, C.W. Curtis and D.C. Cronauer Energy Fuels 1992, 6(4), 511. 


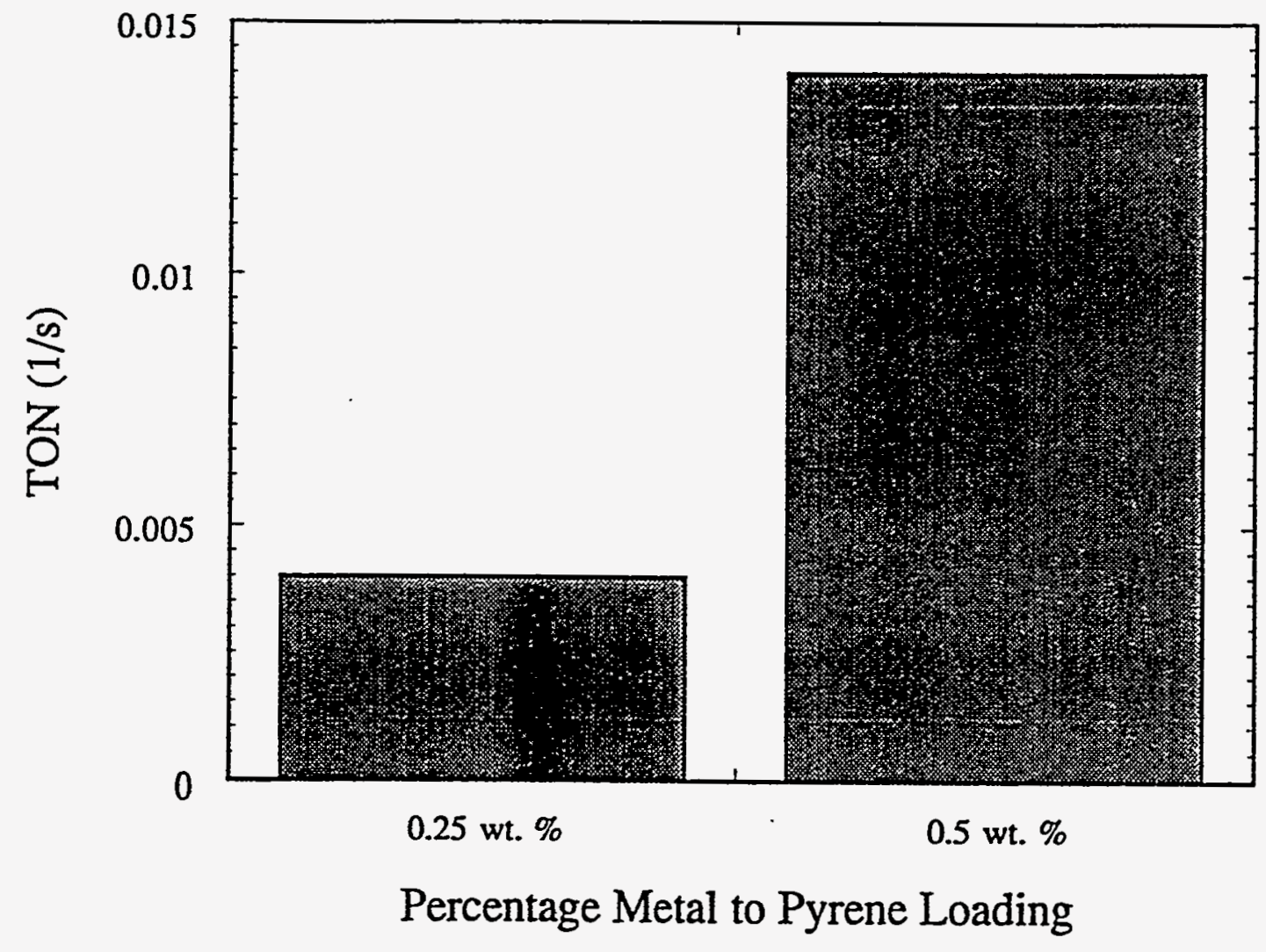

Figure 1: Effect of $\mathrm{Rh}$ concentration on the catalytic hydrogenation of pyrene for 2.4 wt. \% Rh on cube-shaped $\alpha-\mathrm{Fe}_{2} \mathrm{O}_{3}$ materials. TON for cube-shaped $\alpha-\mathrm{Fe}_{2} \mathrm{O}_{3}$, all other iron oxide materials, and $\mathrm{SiO}_{2}$ without $\mathrm{Rh}$ is zero (no products formed). 


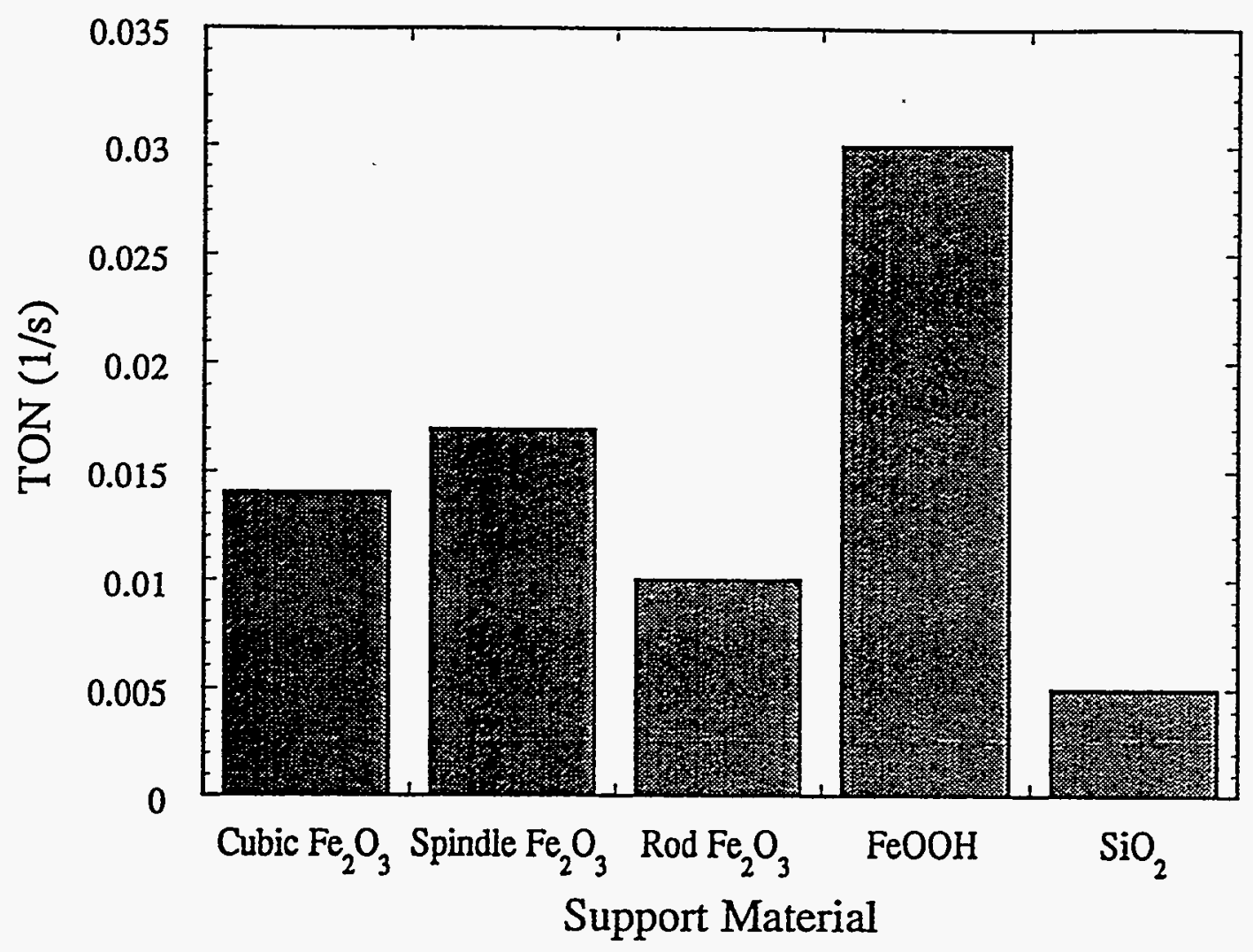

Figure 2: Effect of support composition and morphology on the catalytic hydrogenation of pyrene ( $2.4 \mathrm{wt}$. \% Rh on each support, $0.5 \mathrm{wt}$. \% Rh to pyrene). $\beta$ FeOOH supported materials are roughly two times more active than $\alpha-\mathrm{Fe}_{2} \mathrm{O}_{3}$ supported materials and six times for active than $\mathrm{SiO}_{2}$ supported materials. 


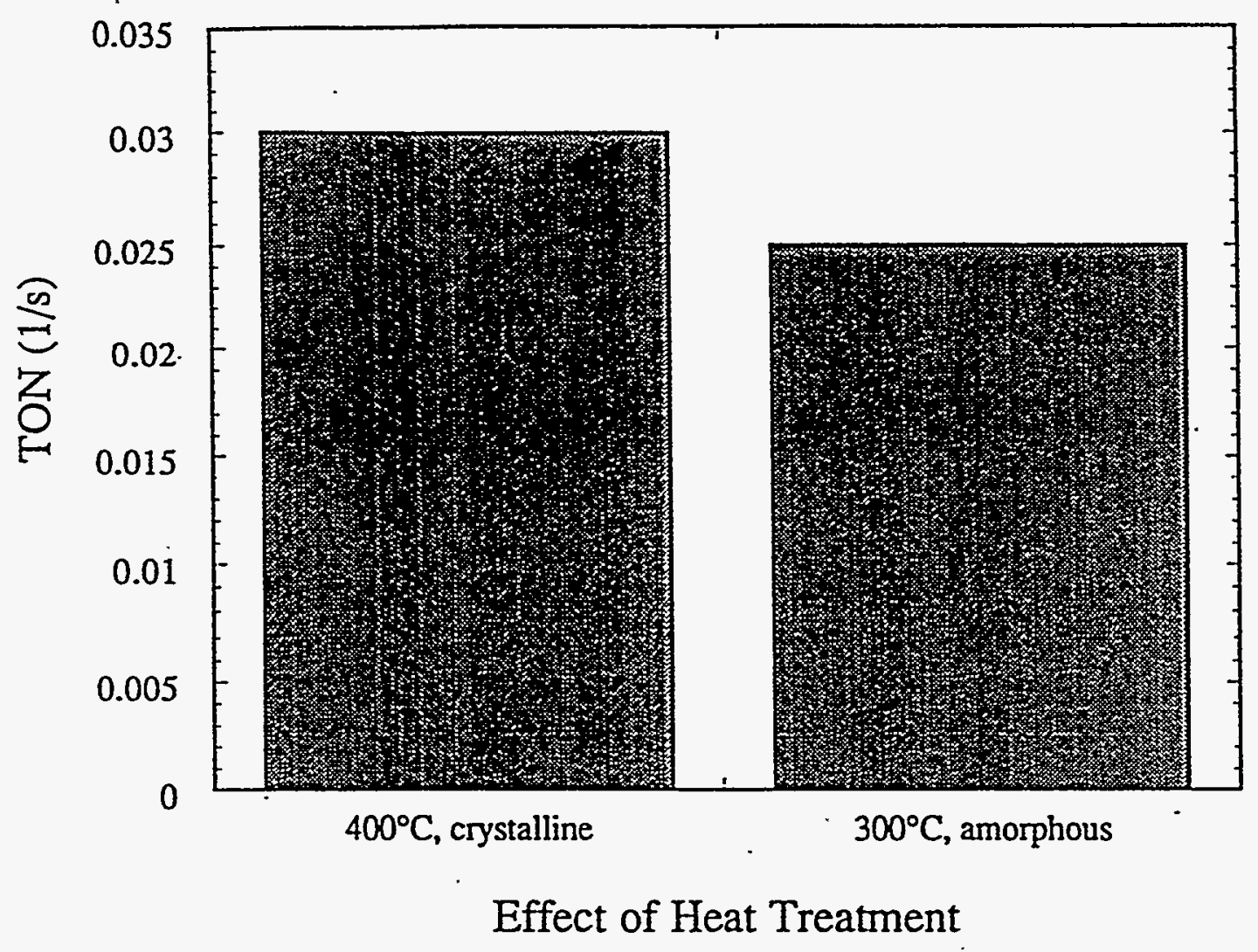

Figure 3: Effect of heat treatment on synthesis of $\beta$-FeOOH supported catalysts (2.4 wt. \% Rh on each support, $0.5 \mathrm{wt}$. \% Rh to pyrene). Catalytic activity decreases slightly with the crystalline to amorphous change in the support material. The crystalline material is synthesized in vacuo at $400^{\circ} \mathrm{C}$ while the amorphous material is synthesized at $300^{\circ} \mathrm{C}$. 


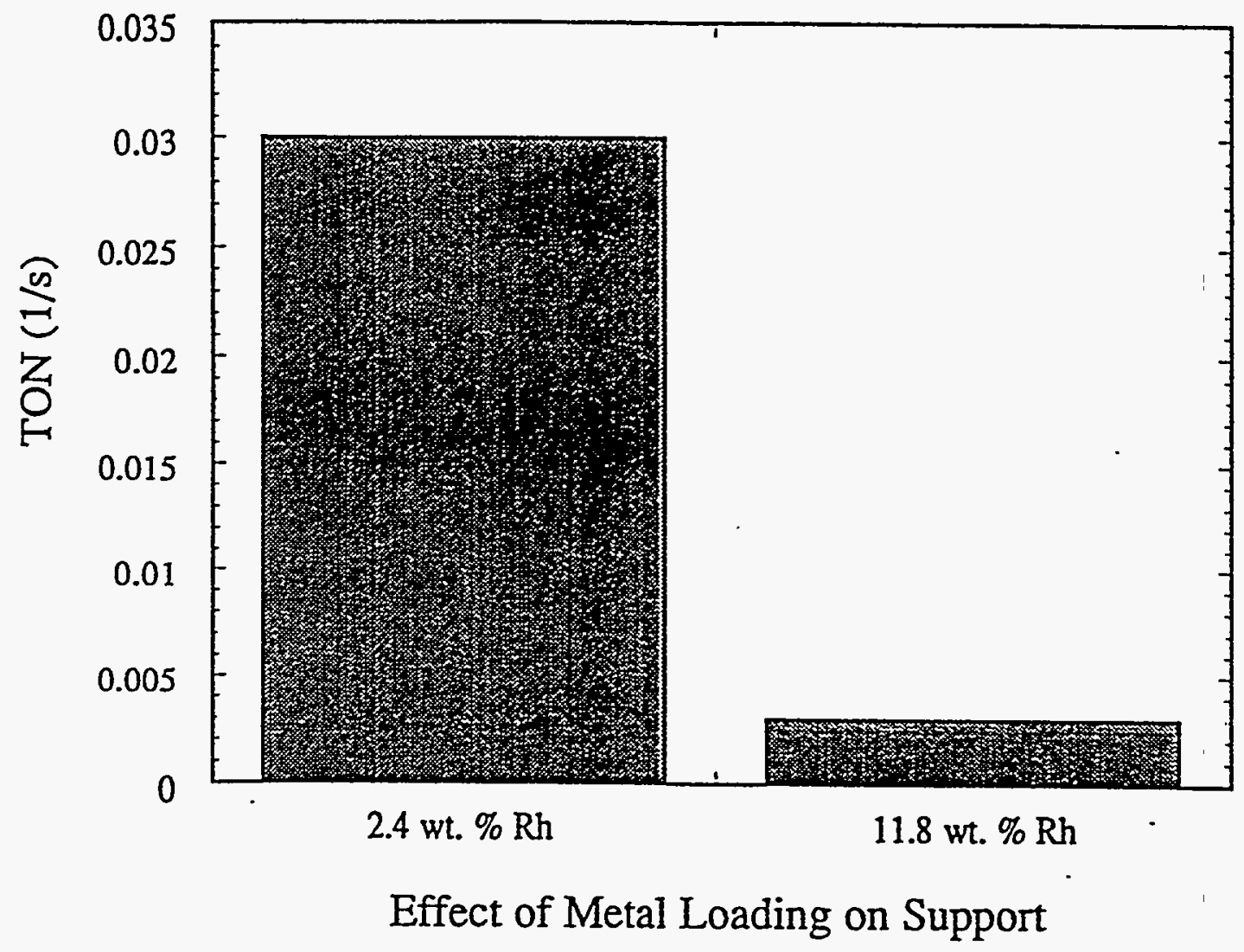

Figure 4: Effect of metal loading on the $\beta$-FeOOH support (at constant metal concentration in the reaction) on catalytic hydrogenation of pyrene. The material with high metal loadings shows a marked decrease in activity due to loss of dispersion of the metal. 


\title{
Chapter 4
}

\section{Encapsulation of Gold Nanoclusters in Silica Materials via an Inverse Micelle / Sol-Gel Synthesis}

\begin{abstract}
Nanometer sized gold particles were encapsulated in the micropores of xerogels and aerogels. The synthesis involves the sequential reduction of a gold salt followed by sol-gel processing in an inverse micelle solution. The inverse micelle solution solubilizes the metal salt and provides a micro-reactor for the nucleation, growth, and stabilization of the nanometer sized clusters. Hydrolysis and condensation of an added siloxane precursor produces a wet gel embedding the particles. Characterization of the particle size and composition and the particle growth process was completed with transmission electron microscopy (TEM), electron diffraction, and UV-visible absorption spectrometry. Characterization of the gel surface areas was completed with $\mathrm{N}_{2}$ porosimetry. Material properties determined as a function of the gel precursor (TEOS vs. a pre-hydrolyzed form of TEOS), the water to gel precursor reaction stoichiometry, and surfactant concentration are discussed in terms of the unique solution chemistry occurring in the micro-heterogeneous inverse micelle solutions.
\end{abstract}

\section{Introduction}

Colloidal sized metal and semiconductor particles with diameters of $1-20 \mathrm{~nm}$ (nanoclusters) are of current interest because they mark a material transition range between molecular and bulk properties. With decreasing colloid size, bulk properties are lost as the continuum of electronic states breaks down (i.e. quantum size effects) and as the fraction of surface atoms becomes large. There are a number of ways to synthesize metal particles with diameters between 1-20 $\mathrm{nm}^{1-3}$. All synthesis routes include nucleation, growth, and stabilization of the particles and attempt to control particle size, size distribution, chemical composition, and structure. Chemical or photolytic reduction ${ }^{46}$, thermal decomposition ${ }^{7.9}$, and vapor phase condensation ${ }^{10-12}$ of metal salts and organometallic reagents initiates 
nucleation and growth. Controlling double layer forces with buffers and electrolytes ${ }^{13.14}$ and the use of steric stabilizing agents such as polymers ${ }^{15-24}$, surfactants ${ }^{25 \cdot 34}$, and bulky ligand appendages ${ }^{35-42}$ stabilize the particles in solution.

A common nanocluster synthesis technique is the reduction of metal salts in inverse micelle solutions ${ }^{25-34}$. Inverse micelles are solution structures formed by the self-assembly of surfactants in apolar solvents (i.e. toluene, alkanes). Surfactants possess two distinct moieties, a hydrophilic head group and a hydrophobic tail group, and they self-assemble in apolar solvents so that the hydrophilic head groups shield themselves from the oleic surroundings $\mathrm{s}^{43}$. The relatively polar head group regions solubilize and confine added metal salts and act as reaction cages when a reducing agent is introduced. Ultra-small, monodispersed particles sterically stabilized in solution by surfactant are formed. Advantages of the inverse micelle synthesis technique include mean particle size control and compositional variety. Particle size control results from regulation of the nucleation site size (inverse micelle size) and the particle growth rate (material exchange rate between inverse micelles). A variety of metals and bimetallics are produced by choosing different metals salts and mixtures of metal salts. Unfortunately, the presence of surfactant hinders potential applications (i.e. catalysis), and removal of the surfactant causes immediate flocculation of the particles with loss of the unique and interesting material properties.

Our goal is to isolate nanoclusters and preserve their unique properties without the presence of any stabilizing agents that attach themselves to the particle surface. We have developed sol-gel processing in inverse micelle, nanocluster solutions to encapsulate or sterically entrap nanoclusters in the micropores of xerogels and aerogels. The synthesis is a sequential reduction of a metal salt and sol-gel processing of an added siloxane precursor in an inverse micelle solution. Sol-gel processing of porous silica gels in polar solvents has been used to encapsulate a variety of large molecules including laser dyes, photochromics, and proteins ${ }^{4-50}$. Sol-gel encapsulation involves polymerization of a gel precursor, usually tetramethoxysilane or tetraethoxysilane, to build the silica gel structure (host) around the dopant (guest). Steric entrapment occurs when the gel pore size is comparable to the dopant size. Also, nanometer sized gold particles have been stabilized with a silica coating using silane coupling agents as surface primers ${ }^{51}$. The product of this work includes nanoclusters in a silica monolith that remain small, of one size, and highly dispersed even after the removal of the surfactant. 
Other works involving sol-gel processing in surfactant, apolar solvent solutions use high surfactant or alcohol concentrations and are not suitable for monodisperse cluster formation $^{52-58}$. We have established an entirely new system with low surfactant concentrations and no alcohol. The inverse micelle solutions and precursor salt solutions are characterized by small angle x-ray scattering (SAXS). After chemical reduction of the metal salt and hydrolysis and condensation of an added siloxane precursor, cluster size, chemical composition, and the cluster growth process are characterized by transmission electron microscopy (TEM), electron diffraction, and UV-visible absorption spectrometry. Gel surface areas are determined by $\mathrm{N}_{2}$ porosimetry. Material properties are determined as a function of the gel precursor (TEOS vs. the pre-hydrolyzed form of TEOS), the water to gel precursor reaction stoichiometry (molar ratio $=4: 1,3: 1,2: 1$, and 1:1), surfactant concentration, drying mechanism, and process washing with hexanol.

We are currently interested in nanocluster-gel materials as catalysts. Metal-gel matrixed materials have been extensively studied as catalysts, because high surface area, high porosity gels act to disperse the active metal, minimize mass transfer and pore diffusion limitations, and promote activity through tunable surface acidities. Traditionally, atomic metals are added to gels when salts are ion-exchanged with terminal hydroxyl groups on the gel $^{59.60}$, through impregnation into the gel backbone during gel processing ${ }^{61.64}$, or through the

use of chelating agents ${ }^{65,66}$. Clusters are formed later through chemical, thermal, and photolytic reduction. No current synthesis method prevents metal particle sintering under robust catalytic conditions, however. Our work is the first where formation of clusters proceeds formation of the gel, thus resulting in encapsulation and potentially new advantages. Clusters are trapped in the gel pores of comparable size limiting the modes of particle sintering. The advantages of the inverse micelle technique are retained including formation of ultra-small, monodisperse, highly dispersed particles, and particle size and composition control (i.e. various metals, mixtures of different metals, alloys, layered particles, metal oxides, and metal sulfides).

\section{Experimental}

Materials: The surfactant didodecyldimethylammonium bromide (DDAB), apolar solvent toluene ( $99.9+\%$ purity), reducing agent lithium borohydride in tetrahydrofuran ( $2 \mathrm{M})$, gold trichloride, tetraethylorthosilicate (TEOS), and a $40 \mathrm{wt}$ \% tetrabutylammonium 
hydroxide (TBAOH) in water solution were purchased from Aldrich and used as delivered. Polydiethoxy siloxane (M.W. $=610 \mathrm{gm} / \mathrm{mol}$ ), an oligomer of TEOS formed by a proprietary pre-hydrolysis reaction, was purchased from United Chemical Technologies of Bristol, PA and used as delivered. Hexanol is used as a washing solvent and is purchased from Aldrich at $99.9+\%$ purity.

Synthesis: Surfactant is added to toluene (1-5 wt. \%) and stirred by hand shaking to form the inverse micelle solution. $\mathrm{AuCl}_{3}(0.001 \mathrm{M})$ and the gel precursor $(0.4 \mathrm{M})$ are added, and the solution is stirred until the salt is fully solubilized. A gold colored transparent solution is formed. The $\mathrm{LiBH}_{4} / \mathrm{THF}$ solution is injected into the salt precursor solution under rapid stirring so that the $\left[\mathrm{BH}_{4}^{-}\right]:\left[\mathrm{Au}^{3+}\right]=3: 1$. The gold solution immediately turns dark purple. The $40 \mathrm{wt}$ \% TBAOH in water solution is added 1 to 5 minutes afterwards, and gelation time is marked when the solution no longer flows under gravity. A deep purple, viscous gel is formed. The water to gel precursor molar ratio is studied as an experimental variable and is set at 1:1, 2:1, 3:1, and 4:1. Two different gel precursors are tested: TEOS and a pre-hydrolyzed form of TEOS. The gels are aged for fourteen days prior to drying under ambient conditions to form xerogels or under supercritical $\mathrm{CO}_{2}$ extraction to form aerogels. Some samples were washed to remove surfactant and reaction by-products by passing warm hexanol over the dried, crushed gels through an aspirator funnel. Samples discussed in 'Results' were not washed unless indicated.

Characterization: SAXS was completed at the Small-Angle Scattering Center at the University of New Mexico. A $12 \mathrm{~kW}$ rotating anode source was used with at Kratky camera and an M-Braun linear position sensitive detector. A Ni filter was used as a monochromator, and the samples were sealed in quartz capillary tubes. Particle size and composition in the colloidal solutions were characterized with transmission electron microscopy (TEM) and electron diffraction. These tests were performed with a $300 \mathrm{keV}$ Phillips CM30 electron microscope. Dried, crushed gels were dispersed over a holey carbon substrate. UV-visible absorption spectroscopy was completed with a Hewlett Packard 8452A Diode Array spectrophotometer. Quartz cuvettes were used in liquid samples, and gels were sliced and mounted on quartz microscope slides. BET surface area analysis of the crushed xerogels and aerogels was carried out with a Quantachrome Autosorb-6 surface analysis apparatus. 


\section{Results}

Small angle $\mathrm{x}$-ray scattering was used to characterize the inverse micelle solutions and the effect of the addition of the gold salt and gel precursors to the solution structure. SAXS of DDAB/toluene inverse micelle solutions, gold salt in DDAB/toluene inverse micelle solutions, and gold salt and TEOS in DDAB/toluene inverse micelle solutions is weak but existent. Scattering curves are flat and bend only at relatively large scattering angles. Guinier analysis indicates the aggregate size, $R_{g}=0.49 \mathrm{~nm}$, remains constant in all three cases (Figure 1). No small angle x-ray scattering is observed when only TEOS is added to DDAB/toluene mixtures. Finally, scattering and Guinier analysis of gold salt and TEOS solutions after reduction (i.e. after cluster formation, but before hydrolysis and condensation) indicates a cluster size of $R_{\mathrm{g}}=2.34 \mathrm{~nm}$.

Formation of the clusters occurs within seconds after addition of the reducing agent. The gold solutions turn purple, the color indicative of the presence of colloidal sized gold. Gelation occurs in the cluster, surfactant solutions at 1.5, 2.5, 3.3, and 24 hours after the addition of TBAOH/H $/ \mathrm{H}_{2} \mathrm{O}$ for samples with the reaction stoichiometry of $1: 1,2: 1,3: 1$, and 4:1 molar ratio of water to TEOS. The pre-hydrolyzed TEOS precursor samples gel much more quickly compared to the TEOS precursor. With the molar ratio of water to TEOS oligomer = $4: 1$, gelation occurs in 15 minutes. For reaction stoichiometries of $3: 1$ and less, gelation occurs within 5 minutes. The basic water solution is fully solubilized in the inverse micelle solution before gelation. The clusters are embedded in the gels upon gelation. The solutions are transparent and clear (yet colored) at all water concentrations and no cloudiness or indication of phase separation is visibly apparent.

"All wet gels are transparent, homogeneously deep purple, viscous, and undergo little to no synerises (Figure 2). Drying to form xerogels causes the gels to shrink $1 / 3$ to $1 / 2$ of their original volume independent of reaction stoichiometry and precursor type. Deep purple monoliths are formed. Drying to form aerogels causes little shrinking in pre-hydrolyzed TEOS gels and shrinks TEOS precursor gels by $1 / 2$ of their original volume. Aerogels appear chalky and light purple, but regain their color when wetted.

The synthesis produces highly dispersed, monodispersed, nanometer sized gold particles embedded in silica matrices (Figure 3). Particle size is tested as a function of reaction stoichiometry, the gel precursor type, the drying procedure, and washed vs. unwashed samples. Particle size is independent of reaction stoichiometry, gel precursor type, 
and the effect of washing (Table 1). The size distribution of a typical sample is relatively narrow (Figure 4). For washed samples, particle size is independent of the drying procedure (xerogels vs. aerogels). It appears that the particles are washed out of samples made with the pre-hydrolyzed form of the TEOS precursor. Few are observed within the gel by TEM. Particle size is roughly twice as large in gels as in pure inverse micelle solutions of DDAB and toluene $e^{67}$. Electron diffraction from the clusters show five rings that index to the presence of FCC-Au. The relative diffraction peak radii for the five rings are 1.0, $0.86,0.61$, 0.52 , and 0.40 . It should be noted that our work with Pd systems indicate $0.01 \mathrm{M}$ metal salt precursor solutions result in approximately $1 \mathrm{wt}$. $\% \mathrm{Pd}$ on $\mathrm{SiO}_{2}$ materials as measured by atomic adsorption spectroscopy.

Monitoring cluster size in solution and in wet gels as a function of time provides information on the growth mechanism and the effect of gelation on particle growth. Size is monitored by measuring the gold plasmon resonance observed by UV-visible spectroscopy. Colloidal sized gold in solution exhibits a plasmon resonance between 500 and $550 \mathrm{~nm}$ depending on particle size. Increasing particle size results in a red shift in the plasmon resonance. Immediately after salt reduction, the gold plasmon resonance occurs at $502 \mathrm{~nm}$ in gel precursor, $\mathrm{DDAB}$, and toluene solutions. The plasmon resonance red shifts to $509 \mathrm{~nm}$ after three hours and stabilizes at $526 \mathrm{~nm}$ after seven days (Figure 5). If gelation is initiated to a new sample by adding TBAOH/water to the solution, the gold plasmon resonance is 518 $\mathrm{nm}$ and $527 \mathrm{~nm}$ after three hours and after seven days, respectively (Figure 5). The particles slowly grow after the reduction before stabilizing in both un-gelled and gelled samples. The particle growth mechanism is faster, but the final particle size is unaffected by the gelation process (assuming constant medium refractive indices between ungelled and gelled samples).

Surface areas as determined by $\mathrm{N}_{2}$ porosimetry are strongly dependent on the reaction stoichiometry, surfactant concentration, and gel precursor type. With some exceptions, aerogel surface areas increase as the molar ratio of water to gel precursor increases and as surfactant concentration decreases (Figures 6 and 7). Aerogels formed with pre-hydrolyzed TEOS precursor exhibit higher surface areas than when formed with TEOS (compare Figures $6 a$ and $7 a)$. When gold is embedded in the silica matrices, the surface areas increase and follow the same trend with reaction stoichiometry (Figures $6 \mathrm{~b}$ and $7 \mathrm{~b}$ ). To determine if surfactant changes the reaction chemistry and thus the material properties or obstructs the silica surface, the materials were washed with warm hexanol. Surface areas increase and 
follow similar trends with reaction stoichiometry (Figures $6 \mathrm{~b}$ and $7 \mathrm{~b}$ ). TEOS xerogels exhibit negligible surface areas (not shown). Pre-hydrolyzed TEOS xerogels (not shown) exhibit similar surface areas and trends as do pre-hydrolyzed TEOS aerogels (Figure 7a). The silica matrices are mesoporous with pore diameters ranging from $5-30 \mathrm{~nm}$.

\section{Discussion}

SAXS indicates that inverse micelles formed in DDAB/toluene solutions are small $\left(R_{g}=0.49 \mathrm{~nm}\right)$ with only a few surfactant molecules forming each aggregate structure. SAXS also indicates that the addition of gold salt or the addition of both gold salt and TEOS have no effect on aggregate size. However, SAXS is not observed when only TEOS is added to inverse micelle solutions. .TEOS alone causes dissolution of the surfactant aggregate structures. TEOS is miscible in toluene, but relatively polar. The driving force for surfactant aggregation decreases as the gel precursor acts as a co-solvent for toluene and the polar moieties of the surfactant. It is likely that there is some effect of TEOS on gold salt inverse micelle structures, and the effect is offset by the salt or not detected due to weak scattering.

The size of particles formed by the reduction of metal salts in typical inverse micelle solutions depends on the inherent size of the inverse micelles and the material exchange rate between inverse micelles. Both micelle size and the material exchange rate depend on experimental parameters like the surfactant/solvent system, the addition of water which swells the inverse micelles, and the salt to surfactant ratio. Particle size control is often complicated but feasible. Our work with the TEOS, DDAB, and toluene system establishes three results: (1) the reduction reaction in TEOS inverse micelle solutions and in gelled samples produces particles roughly two times larger than particles formed in only DDAB/toluene mixtures ${ }^{67}$, (2) the gelation has little effect on the final particle size as determined by the plasmon resonance, but does influence the initial growth rate, and (3) the particle size is not dependent on the gel precursor type, the reaction stoichiometry, or the drying method.

The effect of TEOS on the inverse micelle structure and the cluster growth and stabilization processes is the likely cause for the increased particle size in the mixtures studied here compared to particles synthesized in traditional DDAB/toluene mixtures. Cluster nucleation and growth is sustained within the inverse micelles. A change in micelle structure and the growth and stabilization process is inferred from the effects of TEOS on the 
surfactant aggregation phenomena as detected by SAXS. With the addition of the base and water solution, hydrolysis and condensation of the TEOS starts. The presence of water and the further production of water and alcohol in the reactions further destabilizes the inverse micelle structure and causes the clusters to grow at a faster rate as evidenced by the UVvisible absorption spectra. However, the final particle size is unchanged in gelled systems compared to ungelled systems. Apparently, an upper bound in the stabilization process is reached.

Some of the variables adjusted in this study to control material properties include the gel precursor type, the water to gel precursor reaction stoichiometry, and the drying process (xerogels vs. aerogels). The precursor type and drying process would have no direct effect on particle size control and none was observed. More typical methods to control particle size include, the surfactant/solvent system, the metal salt to surfactant ratio, and water content. Different surfactant/solvent systems were explored in this study (Polyoxyethylene (6) nonyl phenyl in cyclohexane and tetraethyleneglycol mono n-dodecyl ether in octane), but results were less fruitful. Silica particles resulted, or phase separation occurred with only the water rich phase gelling ${ }^{68}$. Salt to surfactant ratios were not varied in this study. We attempted to control particle size through the use of water content. No particle size control is apparent in this effort most likely due to the destabilization of the inverse micelle structure in the presence of TEOS and the hydrolysis and condensation by-products. However, the principles of particle size control remain in this methodology and further effort is required.

Despite the lack of particle size control, the role of the surfactant is clear. The inverse micelles act to solubilize the metal salt, stabilize the clusters against growth, and solubilize the $\mathrm{TBAOH} / \mathrm{H}_{2} \mathrm{O}$ mixture during gelation. If surfactant is not used, little $\mathrm{AuCl}_{3}$ is solubilized in TEOS, toluene mixtures $(<<0.001 \mathrm{M})$. Reduction with $\mathrm{LiBH}_{4}$ results in large particles that are only stable for a few hours. Finally, introduction of the $\mathrm{TBAOH} / \mathrm{H}_{2} \mathrm{O}$ mixture results in immediate precipitation of the clusters, phase separation of a water rich and an oil rich phase, and no gelation.

In traditional sol-gel chemistry, the relative rates of hydrolysis and condensation as effected by sol-gel processing parameters like acid vs. base catalyzed reactions and the $\mathrm{H}_{2} \mathrm{O}: \mathrm{Si}$ ratio control the final gel properties ${ }^{6,70}$. In general, hydrolysis dominates under acidcatalyzed, low $\mathrm{H}_{2} \mathrm{O}$ :Si ratio conditions and weakly branched, polymeric sols and gels are produced. Condensation dominates under base-catalyzed, high $\mathrm{H}_{2} \mathrm{O}: \mathrm{Si}$ ratio conditions to 
produce highly condensed particulate sols and gels. As condensation rates increase, gelation time and gel surface areas generally decrease.

For gels prepared with TEOS in inverse micelle solutions as the $\mathrm{H}_{2} \mathrm{O}$ :Si ratio increases, gel times and surface areas increase in direct contradiction to the above stated conventional wisdom. We propose that the mechanism of gelation in the cluster solutions depends on the relative solubilities of the reactants in various 'areas' of the microheterogeneous solutions. TEOS is soluble in toluene, and DDAB is soluble in TEOS. Thus, TEOS most likely exists within the inverse micelle and throughout the solution. Decreased aggregation of DDAB in toluene with the introduction of TEOS as detected by SAXS supports this hypothesis. Added water is only soluble within the inverse micelle. Thus, it is likely that gelation occurs mostly at the surfactant interface. Two important results stem from this hypothesis. First, the effective $\mathrm{H}_{2} \mathrm{O}: \mathrm{Si}$ ratio is much higher than expected from the set $\mathrm{H}_{2} \mathrm{O}$ :Si recipe resulting in high condensation rates. As a result, gels are formed in this study even at low $\mathrm{H}_{2} \mathrm{O}$ :Si recipes, and surface areas of TEOS gels are low. Second, with increasing $\mathrm{H}_{2} \mathrm{O}$ :Si ratio, the $\mathrm{H}_{2} \mathrm{O}: \mathrm{DDAB}$ ratio increases. Increasing the $\mathrm{H}_{2} \mathrm{O}$ :DDAB leads to larger, more polydisperse inverse micelles and a subsequent decrease in the surface area of reaction. Increasing the $\mathrm{H}_{2} \mathrm{O}: \mathrm{Si}$ recipe actually leads to a decrease in the effective $\mathrm{H}_{2} \mathrm{O}: \mathrm{Si}$ ratio, and thus, lower condensation rates and the observed higher surface areas and faster gel times. While the proposed gelation mechanism explains the existing data, it is clearly not universally applicable. Monoliths are not formed in other inverse micelles systems, and the use of other structured surfactant phases produce periodic silica phases ${ }^{71 \cdot 73}$.

Gel surface areas increase with decreasing surfactant concentration. This result is consistent with our proposed mechanism. As surfactant concentration decreases, the $\mathrm{H}_{2} \mathrm{O}$ :DDAB ratio increases resulting in a decrease in the surface area of the reaction. Decreasing surfactant concentration leads to lower effective $\mathrm{H}_{2} \mathrm{O}:$ Si ratios, lower condensation rates, and the observed higher surface areas. It is apparent from the increase in the gel surface area after the surfactant is washed away that surfactant also blocks the available gel surface.

Gelation times are faster and surface areas are higher when the pre-hydrolyzed form of TEOS is used. These results indicate that the condensation rates for the pre-hydrolyzed TEOS precursor reactions are slower than for the TEOS precursor reactions. Steric effects are most likely responsible for the slower condensation rates with the bulkier precursor. 


\section{Conclusions}

We have encapsulated nanometer sized Au particles in the micropores of xerogels and aerogels. The synthesis is a sequential reduction of a gold salt and sol-gel processing in an inverse micelle solution. The inverse micelle solution is used to solubilize the metal salt and provide a micro-reactor for the nucleation, growth, and stabilization of the nanometer sized clusters. Hydrolysis and condensation of an added siloxane precursor produces a wet gel embedding the particles. The presence of gel precursors de-stabilizes the inverse micelle structure resulting in larger particle sizes compared to typical inverse micelle synthesis techniques. Particle size control is complicated by the gel precursor effect on inverse micelle structure and the production of water and alcohol in the hydrolysis and condensation reactions. Finally, a unique gelation technique is outlined in these micro-heterogeneous solutions. Gelation occurs across the surfactant interface increasing the effective $\mathrm{H}_{2} \mathrm{O}: \mathrm{Si}$ ratio. Gelation occurs even at low $\mathrm{H}_{2} \mathrm{O}: \mathrm{Si}$ ratios, and condensation rates are high. Sol-gel parameters like the $\mathrm{H}_{2} \mathrm{O}$ :Si ratio and the surfactant concentration have unique and sometimes not intuitive effects on the hydrolysis and condensation rates and the resulting material properties.

\section{Acknowledgments}

The authors thank Thomas Headley of the Electron Microscopy/Metallography Department at Sandia for the TEM's, Edward M. Russick of the Organic Materials Processing Department at Sandia for the supercritical extractions, Duen Wu Hua and Thomas P. Rieker of the University of New Mexico for the SAXS, and Steven K. Showalter of the Solar Thermal Technology Department at Sandia. 


\section{References}

(1) Bradley, J.S. Clusters and Colloids, From Theory to Applications; Schmid, G., VCH Publications, Inc., 1994, Chapter 6.

(2) Steigerwald, M.L.; Brus, L.E. Annu. Rev. Mater. Sci. 1989, 19, 471.

(3) Henglein, A. Chem. Rev. 1989, 89, 1861.

(4) Shen, J.; Hu, Z.; Hsia, Y.; Chen, Y. Appl. Phys. Lett. 1991, 59, 2510.

(5) Takiyama, K. Bull. Chem. Soc. Jpn. 1958, 31, 944.

(6) Turkevich, J.; Stevenson, P.C.; Hillier, J. J. Am. Chem. Soc. Faraday Soc. Disc. 1951, $11,55$.

(7) Brennan, J.G.; Siegrist, T.; Carroll, P.J.; Stuczynski, S.M.; Brus, L.E.; Steigerwald, M.L. J. Am. Chem. Soc. 1989, 111, 4141.

(8) Hoon, S.R.; Kilner, M.; Russell, G.J.; Tanner, B.K. J. of Magnetism \& Magnetic Materials 1983, 39, 107.

(9) Griffiths, C.H.; O’Horo, M.P.; Smith, T.W. J. Appl. Phys. 1979, 50, 7108.

(10) Lin, S.T.; Franklin, M.T.; Klabunde, K.J. Langmuir 1986, 2, 259.

(11) Kimura, K.; Bandow, S. Bull. Chem. Soc. Jpn. 1983, 56, 3578.

(12) Francis, C.G.; Huber, H.; Ozin, G.A. Inorg. Chem. 1980, 19, 219.

(13) Quinn, M.; Mills, G. J. Phys. Chem. 1994, 98, 9840.

(14) Trivino, G.L.; Klabunde, K.J.; Dale, E.B. Langmuir 1987, 3, 986.

(15) Ng Cheong Chan, Y.; Schrock, R.R.; Cohen, R.E. Chem. Mater. 1992, 4, 24.

(16) Cummins, C.C.; Schrock, R.R.; Cohen, R.E. Chem. Mater. 1992, 4, 27.

(17) Ishizuki, N.; Torigoe, K.; Esumi, K.; Meguro, K. Colloids and Surfaces 1991, 55, 15.

(18) Mahler, W. Inorg. Chem. 1988, 27, 435.

(19) Wang, Y.; Suna, A.; Mahler, W.; Rasowski, R.J. J. Chem. Phys. 1987, 87, 7315.

(20) Kuczynski, J.P.; Milosavljevic, B.M.; Thomas, J.K. J. Phys. Chem. 1984, 88, 980.

(21) Mau, A.W.H.; Huang, C.B.; Kakuta, N.; Bard, A.J.; Campion, A.; White, M.J.; Webber, S.E. J. Am. Chem. Soc. 1984, 106, 6537.

(22) Fojtik, A.; Weller, H.; Koch, U.; Henglein, A. Ber. Bunsenges. Phys. Chem. 1984, 88, 969.

(23) Meisssner, D.; Memming, D.; Kastening, B. Chem. Phys. Lett. 1983, 96, 34.

(24) Hess, P.H.; Parker, P.H. J. Appl. Polym Sci. 1966, 10, 1915.

(25) Torigoe, K.; Esumi, K. Langmuir 1992, 8, 59.

(26) Esumi, K.; Shiratori, M.; Ishizuka, H.; Tano, T.; Torigoe, K.; Meguro, K. Langmuir $1991,7,457$.

(27) Zhao, X.K.; Xu, S.; Fendler, J.H. Langmuir 1991, 7, 520.

(28) O'Sullivan, E.C.; Patel, R.C.; Ward, A.J.I J. Coll. Inter. Sci. 1991, 146, 582.

(29) Larpent, C.; Brisse-Le Menn, F.; Patin, H. J. Molec. Catal. 1991, 65, L35.

(30) Borgarello, E.; Lawless, D.; Serpone, N.; Pelizzetti, E.; Meisel, D. J. Phys. Chem. 1990, 94, 5048.

(31) Maguro, K.; Torizuka, M.; Esumi, K. Bull. Chem. Soc. Jpn. 1988, 61, 341.

(32) Monserrat, K.; Gratzel, M.; Tundo, P. J. Am. Chem. Soc. 1980, 102, 5527.

(33) Kiwi, J.; Gratzel, M. J. Am. Chem. Soc. 1979, 101, 721.

(34) Chen, J.P.; Sorensen, C.M.; Klabunde, K.J. Phys. Rev. B 1995, 51, 11527.

(35) Nosaka, Y.; Ohta, N.; Fukuyama, T.; Fujii, N. J. Coll. Inter. Sci. 1993, 155, 23. 
(36) Duan, Z.; Hampden-Smith, M.J.; Datye, A.; Nigrey, P.; Quintana, C.; Sylwester, A.P. J. of Catal. 1993, 139, 504.

(37) Folkers, J.P.; Laibinis, P.E.; Whitesides, G.M. Langmuir 1992, 8, 1330.

(38) Zimmermann, F.; Wokann, A. Molec. Phys. 1991, 73, 959.

(39) Becker, C.; Fries, T., Wendelt, K.; Kreibig, U.; Schmid, G. J. Vac. Sci. Technol. B $1991,9,810$.

(40) Hayes, D.; Meisel, D.; Micic, O.I. Collods and Surfaces 1990, 55, 121.

(41) Andrews, M.P.; Ozin, G.A. J. Phys. Chem. 1986, 90, 2929.

(42) Henglein, A. J. Phys. Chem. 1979, 83, 2209.

(43) Langevin, D. Acc. Chem. Res. 1988, 21, 255.

(44) Zusman, R.; Rottman, C.; Ottolenghi, M.; Avnir, D. J. of Non-Crystalline Solids 1990, $122,107$.

(45) Braun, S.; Rappoport, S.; Zusman, R.; Avnir, D.; Ottolenghi, M. Mater. Letts. 1990, 10, 1.

(46) Dunn, B.; Zink, J.I. J. Mater. Chem. 1991, 1, 903.

(47) Yamanaka, S.A.; Nishida, F.; Ellerby, L.M.; Nishida, C.R.; Dunn, B.; Valentine, J.S.;

Zink, J.I Chem. Mater. 1992, 4, 495.

(48) Ellerby, L.M.; Nishida, C.R.; Nishida, F.; Yamanaka, S.A.; Dunn, B.; Selverstone

Valentine, J.; Zink, J.I. Science 1992, 255, 1113.

(49) Avnir, D.; Braun, S.; Ottolenghi, M. Supramolecular Architecture ;1992; Ch. 27.

(50) Wu, S.; Ellerby, L.M.; Cohan, J.S.; Dunn, B.; El-Sayed, M.A.; Selverstone Valentine, J.; Zink, J.I. Chem. Mater. 1993, 5, 115.

(51) Liz-Marzan, L.M.; Giersig, M.; Mulvaney, P. Langmuir 1996, 12, 4329.

(52) Guizard, C.; Larbot, A.; Cot, L.; Perez, S.; Rouviere, J. J. Chim Phys. 1990, 87, 1901.

(53) Guizard, C.; Stitou, M.; Larbot, A.; Cot, L.; Rouviere, J. Mat. Res. Soc. Symp. Proc. 1988, 121, 115.

(54) Murakata, T.; Sato, S., Ohgawara, T.; Watanabe, T.; Suzuki, T. J. Mater. Sci. 1992, 27, 1567.

(55) Friberg, S.E.; Yang, C.C.; Sjoblom, J. Langmuir 1992, 8, 372.

(56) Friberg, S.E.; Yang, C.C. Innovations in Materials Processing Using Aqueous Colloid and Surface Chemistry; Doyle, F.M.; Raghavan, S.; Somasundaran, P.; Warren, G.W.; 1988; pg. 181.

(57) Jones, S.M.; Amran, A.; Friberg, S.E. J. Disp. Sci. and Tech. 1994, 15, 513.

(58) Lessard, R.B.; Wallace, M.M.; Oertling, W.A.; Chang, C.K.; Berglund, K.A.; Nocera, D.G. Mat. Res. Soc. Symp. Proc. 1989, 155, 109.

(59) Tominaga, H.; Ono, Y.; Keii, T. J. of Catal. 1975, 40, 197.

(60) Ikoma, S.; Takano, S.; Nomoto, E.; Yokoi, H. J. of Non-Crystalline Solids 1989, 113, 130.

(61) Morke, W.; Lamber, R.; Schubert, U.; Breitscheidel, B. Chem. Mater. 1994, 6, 1659.

(62) Ishiyama, J.I.; Kurokawa, Y.; Nakayama, T.; Imaizumi, S.; Appl. Catal. 1988, 40, 139.

(63) Lopez, T.; Villa, M.; Gomez, R. J. Phys. Chem. 1991, 95, 1690.

(64) Lopez, T.; Lopez-Gaona, A.; Gomez, R. Langmuir 1990, 6, 1343.

(65) Moon Choi, K.; Shea, K.J. J. Am. Chem. Soc. 1994, 116, 9052. 
(66) Hardee, J.R.; Tunney, S.E.; Frye, J.; Stille, J.K. J. of Polym. Sci.: Part A: Polym. Chem. 1990, 28, 3669.

(67) Wilcoxon, J.P.; Williamson, R.L.; Baughman, R. J. Chem. Phys. 1993, 98, 9933.

(68) As mentioned in the introduction, other inverse micelle solutions have been used in gelation reactions, but surfactant or alcohol concentrations are high. These recipes are unsuitable in our cluster synthesis. Cluster synthesis was not attempted in the other studies. (69) Brinker, C.J.; Scherer, G.W. Sol-Gel Science-The Physics and Chemistry of Sol-Gel Processing; Academic Press: San Diego, 1990.

(70) Ward, D.A.; Ko, E.I. I\&EC Research 1995, 34, 421.

(71) Kresge, C.T.; Leonowicz, M.E.; Roth, W.J.; Vartuli, J.C.; Beck, J.S. Nature 1992, 359, 710.

(72) Monnier, A.; Schuth, F.; Huo, Q.; Kumar, D.; Margolese, D.; Maxwell, R.S.; Stucky, G.D.; Krishnamurthy, M.; Petroff, P.; Firouzi, A.; Janicke, M.; Chmelka, B.F. Science 1993, 261, 1299.

(73) Huo, Q.; Margolese, D.I.; Ciesla, U.; Feng, P.; Gier, T.E.; Sieger, P.; Leon, R.; Petroff, P.M.; Schuth, F.; Stucky, G.D. Nature 1994, 368, 317. 
Table 1. Particle diameters in nanometers as a function of reaction stoichiometry, drying process, precursor type, and hexanol washing.

\begin{tabular}{|c|c|c|c|c|c|c|c|c|}
\hline \multirow{3}{*}{$\mathrm{H}_{2} \mathrm{O}: \mathrm{Si}$} & \multicolumn{4}{|c|}{ AEROGELS } & \multicolumn{4}{|c|}{ XEROGELS } \\
\hline & \multicolumn{2}{|c|}{ TEOS } & \multicolumn{2}{|c|}{$\begin{array}{c}\text { pre-hydrolyzed } \\
\text { TEOS }\end{array}$} & \multicolumn{2}{|c|}{ TEOS } & \multicolumn{2}{|c|}{$\begin{array}{c}\text { pre-hydrolyzed } \\
\text { TEOS }\end{array}$} \\
\hline & washed & $\begin{array}{c}\text { not } \\
\text { washed }\end{array}$ & washed & $\begin{array}{c}\text { not } \\
\text { washed }\end{array}$ & washed & $\begin{array}{c}\text { not } \\
\text { washed }\end{array}$ & washed & $\begin{array}{c}\text { not } \\
\text { washed }\end{array}$ \\
\hline $2: 1$ & $\begin{array}{c}5.8+/- \\
1.3 \\
\end{array}$ & $\begin{array}{c}5.6+1- \\
1.5 \\
\end{array}$ & $\begin{array}{c}5.7+/- \\
1.3 \\
\end{array}$ & N/A & N/A & $\begin{array}{c}6.0+/- \\
1.5 \\
\end{array}$ & N/A & N/A \\
\hline $4: 1$ & $\begin{array}{c}5.6+/- \\
1.1 \\
\end{array}$ & $\begin{array}{c}6.7+/- \\
1.1 \\
\end{array}$ & N/A & N/A & N/A & $\begin{array}{c}6.7+1- \\
2.2 \\
\end{array}$ & N/A & $\begin{array}{c}7.1+/- \\
1.2 \\
\end{array}$ \\
\hline
\end{tabular}




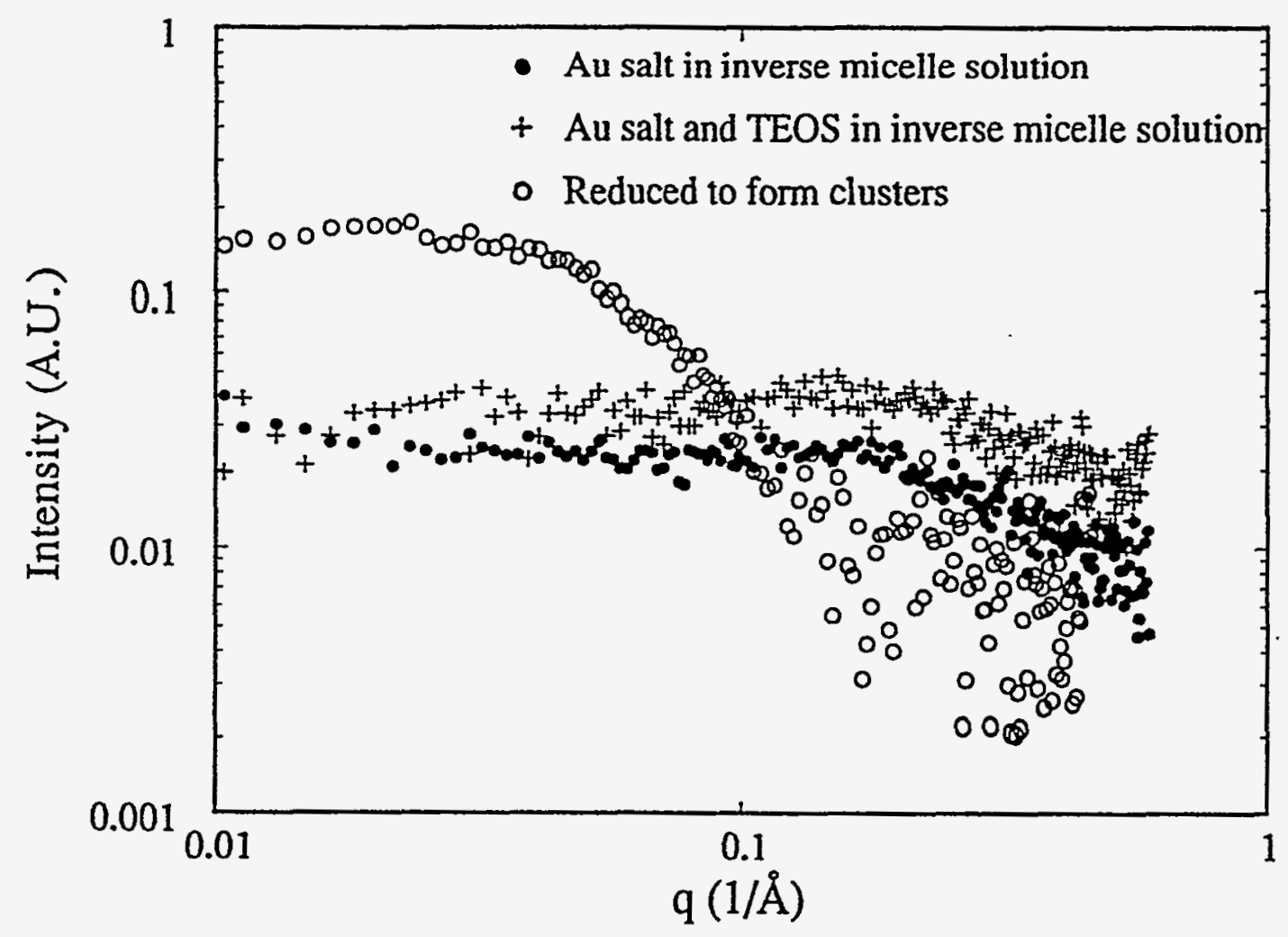

Figure 1. Small angle $x$-ray scattering of DDAB/toluene inverse micelles with a) added $A u$ salt, b) added $A u$ salt and TEOS, c) Au clusters in the presence of TEOS. No SAXS is observed when only TEOS is added to DDAB/toluene. The precursor solutions show small aggregate structures $\left(R_{g}=0.49 \mathrm{~nm}\right)$. The cluster size as detected by SAXS $\left(R_{\varepsilon}=2.34 \mathrm{~nm}\right)$ is slightly smaller than when measured by TEM. $q=(4 \pi / \lambda) \sin \theta$ where $\lambda$ is the wavelength of the radiation and $2 \theta$ is the scattering angle. 


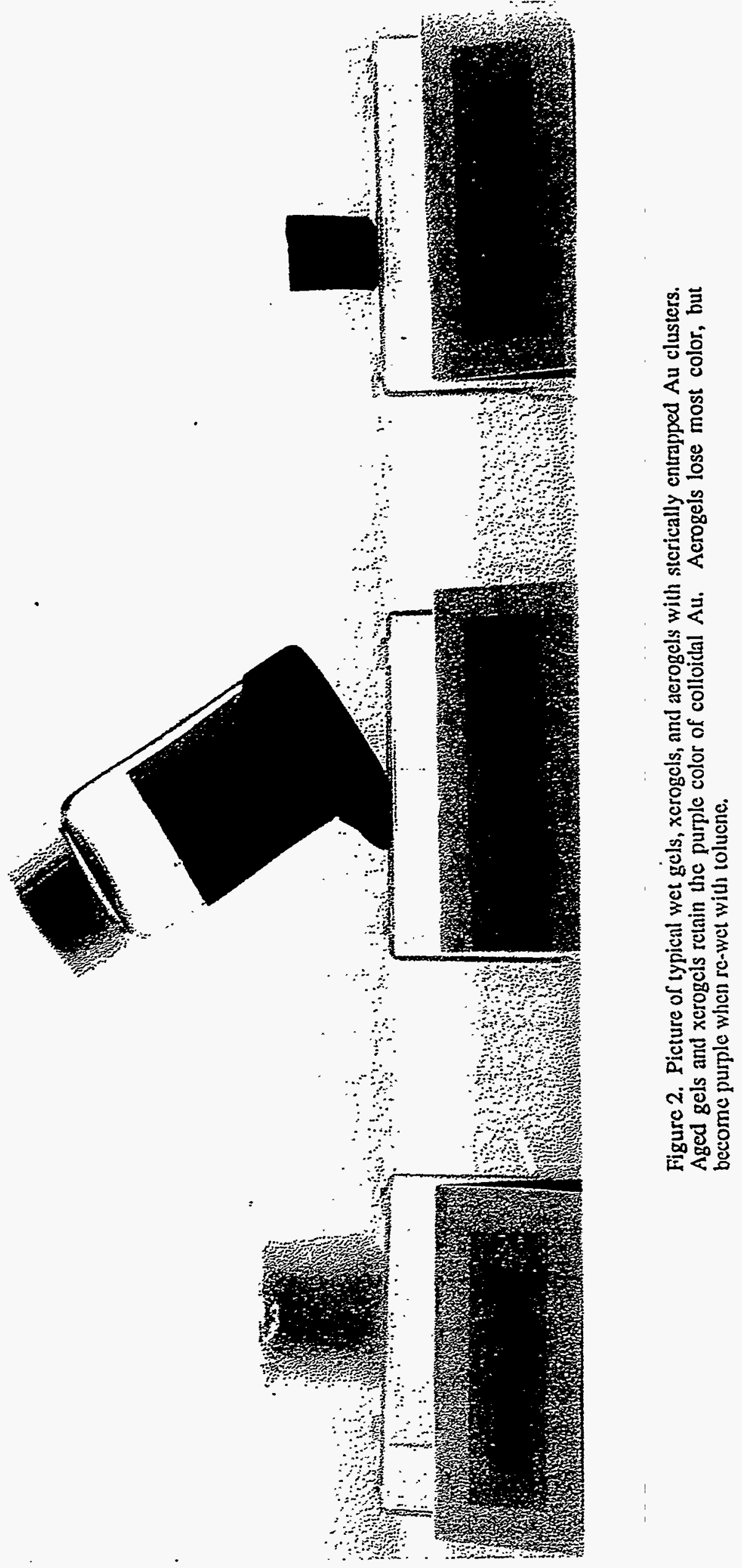




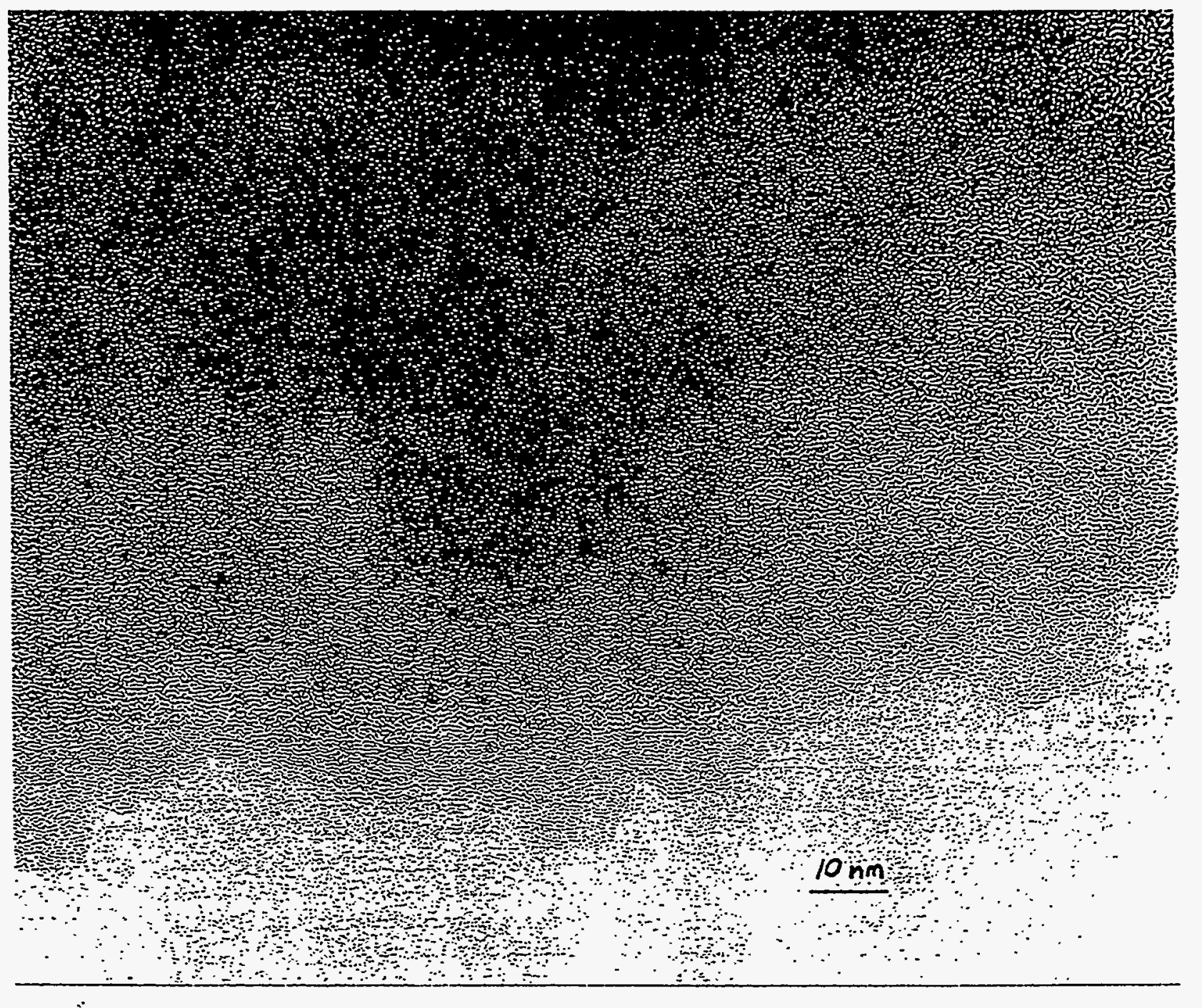

Figure 3. TEM of Au clusters embedded in silica matrix. The particles are $5.7 \mathrm{~nm}$ in diameter and highly dispersed. Particle size is roughly twice that of Au clusters synthesized in traditional DDAB/toluene solutions without TEOS. Particle size is independent of gel precursor type, drying procedure, or the $\mathrm{H}_{2} \mathrm{O}$ :Si ratio. 


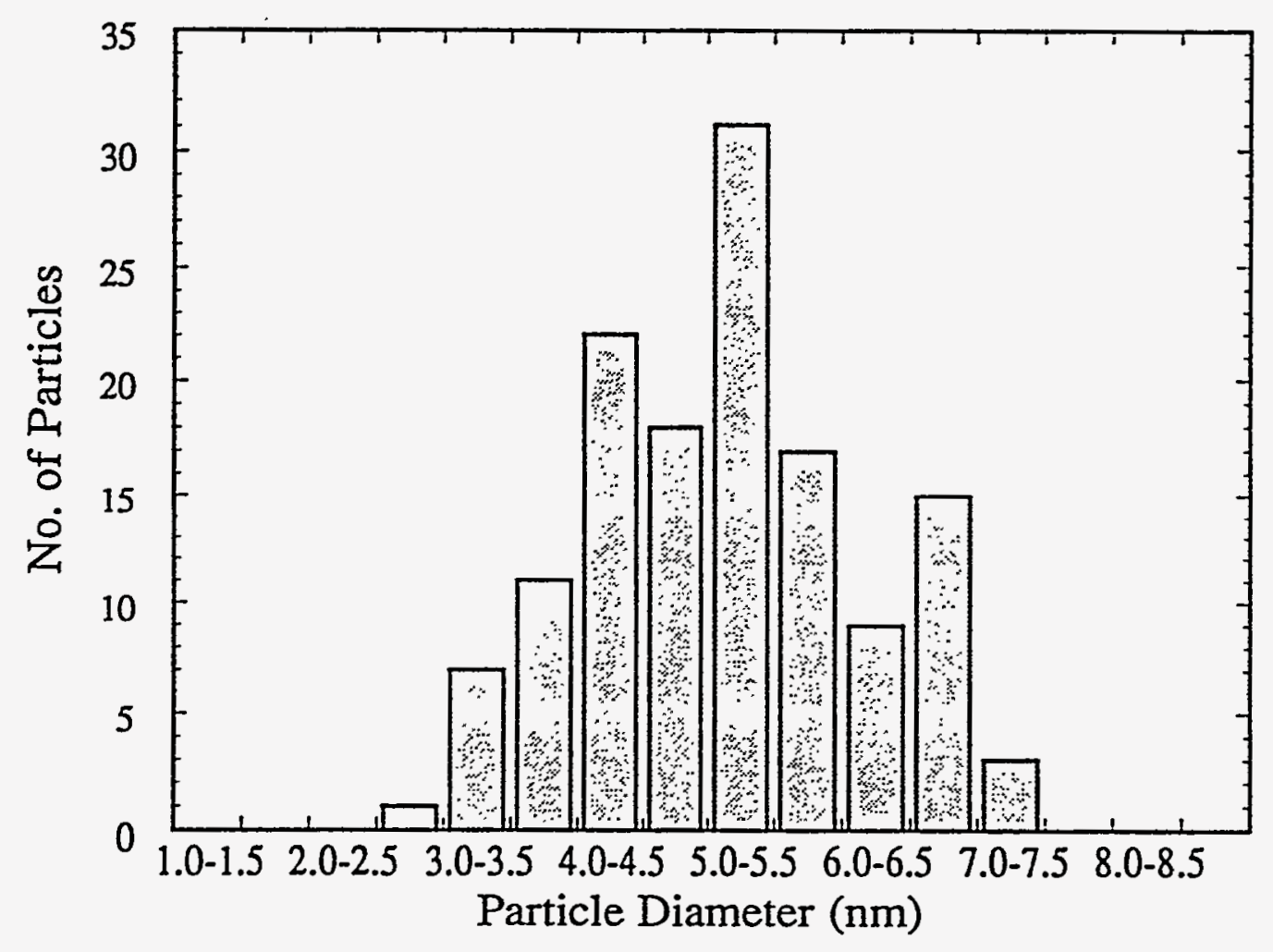

Figure 4. Size distribution as determined by TEM indicating average particle size and monodispersity. 


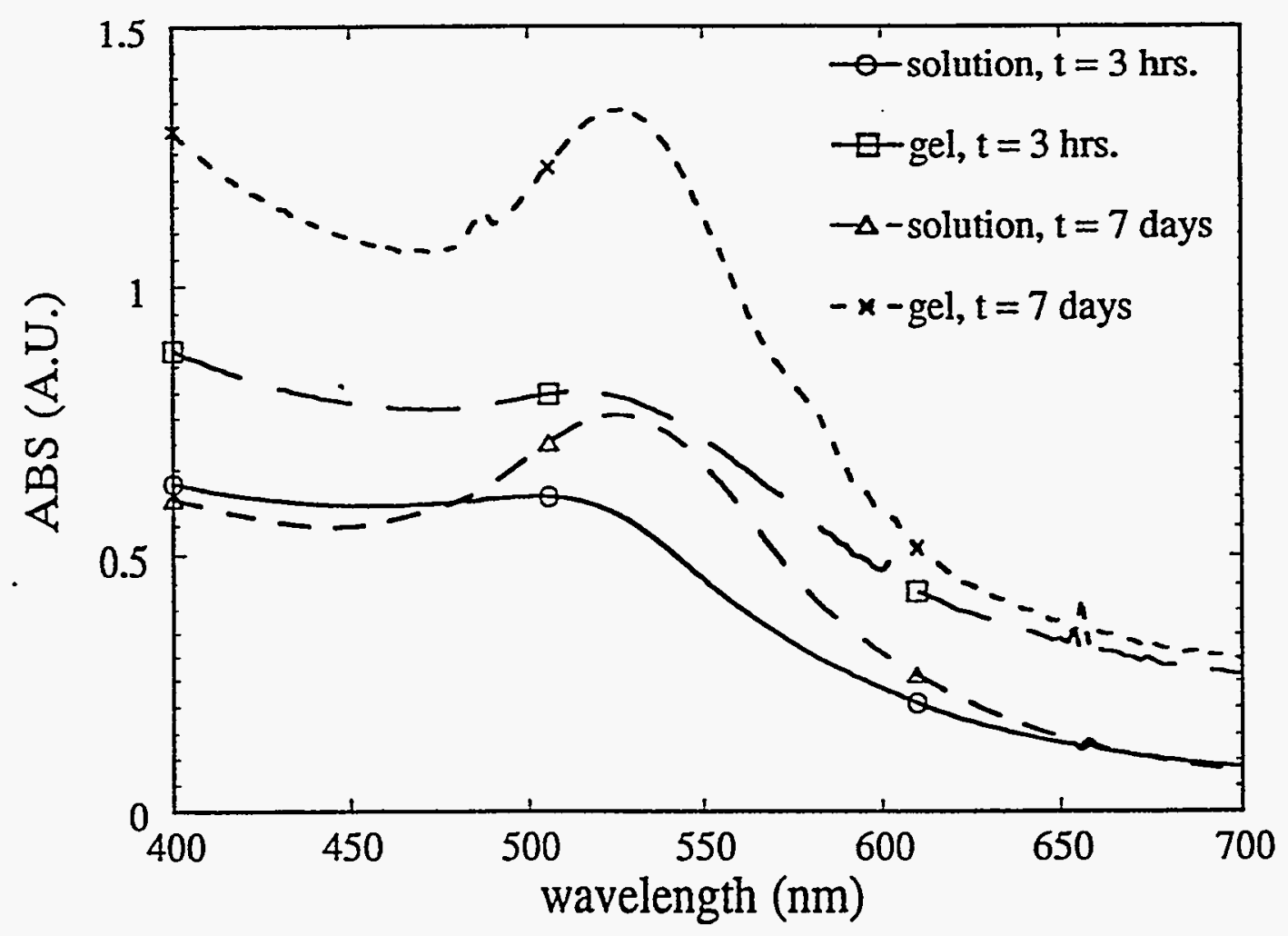

Figure 5. UV-Visible spectrum of Au cluster as a function of time and gelled and ungelled samples. The plasmon resonance red shifts with time as the particles grow. The growth rate is faster in gelled samples, but final particle size is independent of the gelation process. 

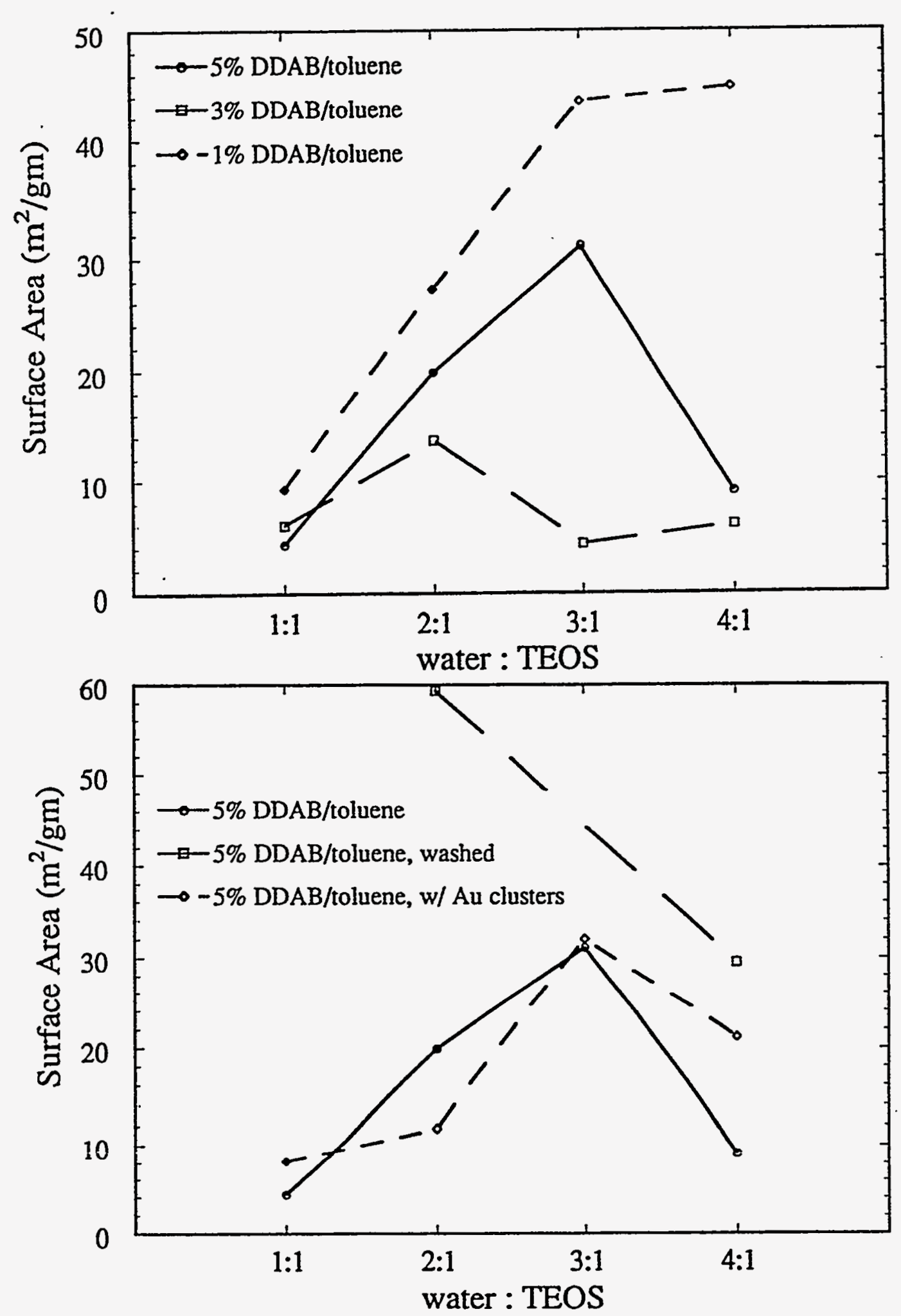

Figure 6. (a) BET surface area's of TEOS aerogels as a function of the $\mathrm{H}_{2} \mathrm{O}: \mathrm{Si}$ ratio and surfactant concentration. Au salt or clusters are not present. The materials are mesoporous. Surface areas generally increase with the reaction stoichiometry and decreasing surfactant concentration. BET surface area's of TEOS xerogels are negligible. (b) BET surface area's of TEOS aerogels as a function of hexanol washing and cluster presence. Surface area's increase when surfactant is washed from the pores. The presence of Au salt or clusters has no effect of gel surface areas. 

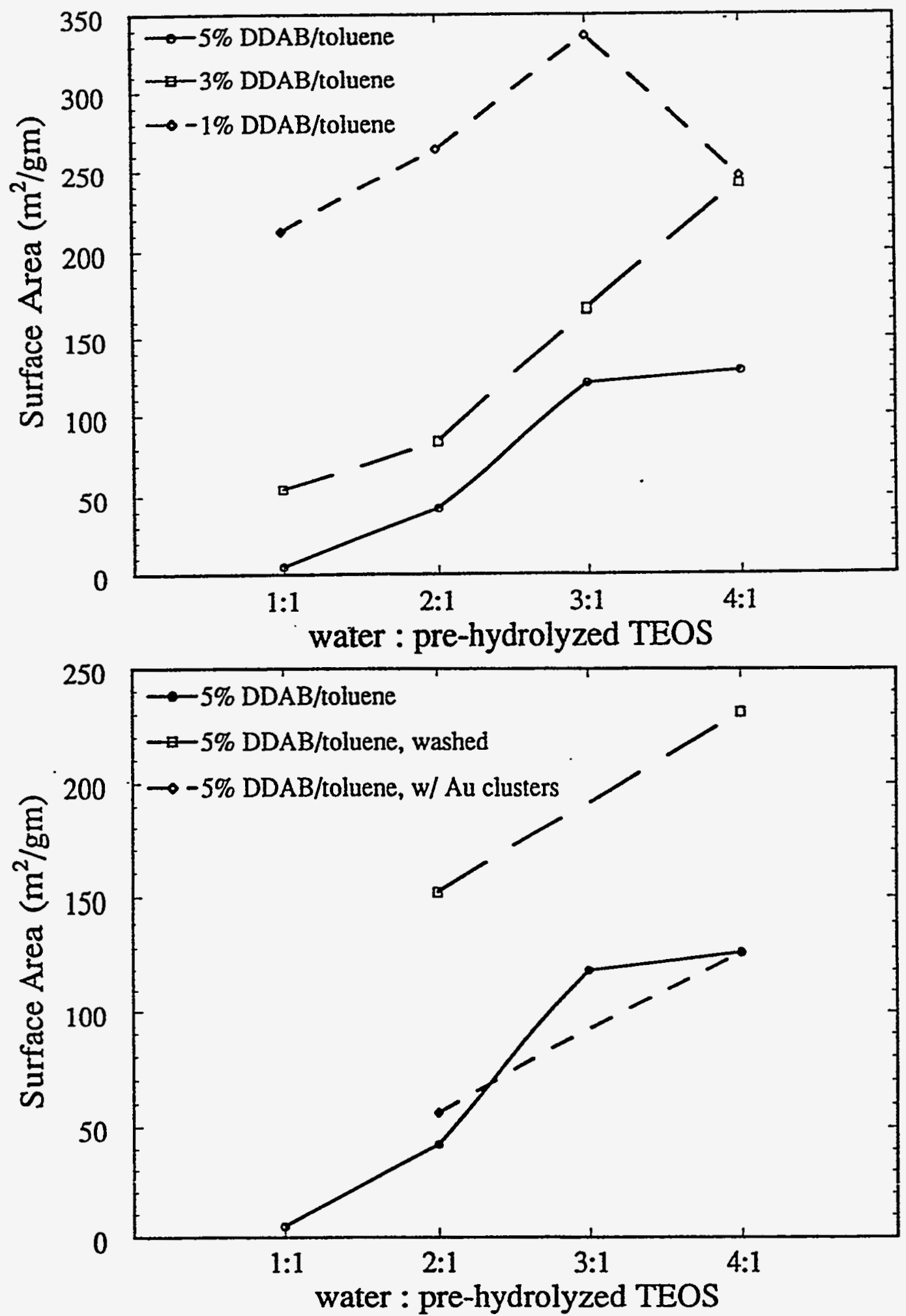

Figure 7. (a) BET surface area's of pre-hydrolyzed TEOS aerogels as a function of the $\mathrm{H}_{2} \mathrm{O}: \mathrm{Si}$ ratio and surfactant concentration. Au salt or clusters are not present. The trends established for TEOS materials are repeated. Surface areas generally increase with the reaction stoichiometry and decreasing surfactant concentration. Pre-hydrolyzed TEOS xerogel surface areas are smaller, but follow similar trends. (b) BET surface area's of prehydrolyzed TEOS aerogels as a function of hexanol washing and cluster presence. The trends established for TEOS materials are repeated. Surface area's increase when surfactant is washed from the pores. The presence of Au salt or clusters has no effect of gel surface areas. 


\title{
Chapter 5
}

\section{Novel Sol-Gel Based Nanocluster Materials as Catalysts in the Dehydrogenation of Propane}

\begin{abstract}
We introduce two novel synthesis strategies to make nanoclusters on silica and alumina supports using inverse micelle technology and sol-gel processing. In the first method, sol-gel chemistry (hydrolysis and condensation of metal alkoxides) is performed in alcohol-free inverse micelle, cluster solutions. In the second method, clusters formed in traditional inverse micelle solutions are allowed to diffuse into pre-existing wet monoliths formed using traditional sol-gel techniques. The different materials produced are characterized and compared with respect to particle size and the substrate properties using $\mathrm{N}_{2}$ porosimetry methods, chemisorption, atomic absorption, and transmission electron microscopy. The effect of calcination on particle and support stability is determined and discussed in terms of the metal loading and the relationship between particle size and pore dimensions. We conclude that the relative sizes of particles and pores has no clear effect on sintering behavior. Sintering appears to be predominately dependent on metal loading suggesting Ostwald ripening as the sintering mechanism, but even this analysis is oversimplified. Finally, we study the materials as catalysts in the selective dehydrogenation of propane with particular interest in stability over time.
\end{abstract}

\section{Introduction}

Nanometer sized metal clusters embedded in sol-gel prepared metal oxide substrates form a unique class of catalytic materials. Sol-gel processing loosely defines the inorganic polymerization reactions of hydrolysis and condensation of metal alkoxides to form solid metal oxides (1). Because many synthesis parameters are available, sol-gel processing offers versatility in final material properties not available by other catalyst synthesis methods (2-5). For instance, by adjusting the oxide precursor type and concentration, the water to precursor reaction ratio, and whether the reactions are acid or base catalyzed, materials with controlled surface areas and pore dimensions are produced. Formation of metal oxide colloidal dispersions (sols) precedes final condensation into monolith, particulate, or thin film gels. 
Also, it is possible to tune molecular composition; $\mathrm{SiO}_{2}, \mathrm{Al}_{2} \mathrm{O}_{3}, \mathrm{TiO}_{2}$, and other pure species, as well as mixed metal oxides are formed. Atomic metals are added later to gels when salts are ion-exchanged with terminal hydroxyl groups $(6,7)$, through impregnation of metal salts into the gel backbone (8-11), or through the use of chelating agents $(12,13)$. Reduced metal clusters are formed by chemical, thermal, and photolytic reduction. Again, the methods are versatile with respect to metal type, and $\mathrm{Pt}, \mathrm{Pd}$, and $\mathrm{Ru}$ are common.

In addition to versatility, metal cluster, sol-gel materials have specific catalytic advantages. For clusters synthesized by impregnation techniques and independent of metal type or support composition, some degree of occlusion of the particles within the metal oxide framework occurs (14-17). As a result, higher resistance to metal sintering is noticed. Also, sol-gel processing parameters have been used to control pore dimensions and particle sizes so that the particles are encapsulated (i.e., the pore size is approximately equal to the particle diameter) (18). Resistance to sintering increases without occlusion of the particles. In one case, however, lower activities were observed due to encapsulation (19). In another study, $\mathrm{Pt} / \mathrm{Al}_{2} \mathrm{O}_{3}$ sol-gel prepared materials showed resistance to coke formation for a number of different support and particle morphologies (20).

In this work, we test the sintering resistance, activity, and selectivity of Pt particles embedded in sol-gel formulated $\mathrm{SiO}_{2}$ and $\mathrm{Al}_{2} \mathrm{O}_{3}$ prepared by several novel synthesis methods. In an earlier study, we synthesized $\mathrm{Au}$ particles in $\mathrm{SiO}_{2}$ by combining inverse micelle techniques with sol-gel processing in nonaqueous, alcohol-free surfactant solutions (21). We have now expanded the synthesis to include the formation of $\mathrm{Pt}$ particles and the formation of $\mathrm{Al}_{2} \mathrm{O}_{3}$ supports. We also develop a second synthesis approach in this paper; namely, inverse micelle synthesized $\mathrm{Pt}$ clusters are encapsulated in traditionally synthesized sol-gel $\mathrm{SiO}_{2}$ supports. The clusters are allowed to diffuse into the pores of a wet alcogel, and drying of the gel encapsulates the particles in the micropores. We describe the synthesis, characterization, and sintering behavior of these materials, and we show activity and selectivity results of these materials tested as catalysts in the dehydrogenation of propane. The synthesis methodologies we present offer the advantages of the inverse micelle technique to make clusters; namely, ultra-small, monodispersed particles of controlled, tunable sizes are formed (22,23). Also, the methodology offers a novel procedure to encapsulate particles in the support by building the metal oxide framework around the clusters. 
The advantages of cluster, sol-gel derived $\mathrm{SiO}_{2}$ and $\mathrm{Al}_{2} \mathrm{O}_{3}$ catalysts address especially important problems in selective dehydrogenation processes. In earlier studies, deactivation $\mathrm{SiO}_{2}$ and $\mathrm{Al}_{2} \mathrm{O}_{3}$ supported metals by surface carbon deposition and/or particle sintering is observed (25-32). Tin as a stabilizer, for instance, is often added to counter deactivation. In particle, sol-gel derived materials, we hope a natural resistance to coke formation and prevention of sintering by encapsulation will relieve some of these problems.

\section{Experimental}

Materials. The following materials were purchased from Aldrich Chemical Co. and were used as delivered: the surfactant didodecyldimethylammonium bromide (DDAB), tetraethylorthosilicate (TEOS), aluminum tri-sec-butoxide (97\%), ethyl acetoacetate (99+\%), platinum (II) chloride ( $98 \%$ ), apolar solvent toluene ( $99.9+\%$ purity), reducing agent lithium borohydride in tetrahydrofuran (2M), and a $40 \mathrm{wt}$. \% tetrabutylammonium hydroxide (TBAOH) in water solution. Acid and base solutions were made from HCL certified $1 \mathrm{~N}$ solution and concentrated $\mathrm{HNO}_{3}$ and $\mathrm{NH}_{4} \mathrm{OH}$ certified ACS plus from Fisher Scientific. Absolute ethyl alcohol was supplied for AAPER alcohol.

Synthesis. The synthesis of nanoclusters via inverse micelle solutions is an established technique $(22,23)$. Metal salts are solubilized within the polar regions of inverse micelles. When a reducing agent is added, nucleation and growth of the metal particles commences. Stabilization of the growth is controlled by the surfactant, and final particle size depends on a number of synthesis variables. The synthesis of $\mathrm{Au}$ particles encapsulated in $\mathrm{SiO}_{2}$ via an inverse micelle, sol-gel combined procedure was described earlier (21, Figure 1). Here, we extend the synthesis to include $\mathrm{Pt}$ particles and $\mathrm{Al}_{2} \mathrm{O}_{3}$ supports (Table 1).

Sequential inverse micelle, sol-gel derived $\mathrm{Pt}_{\mathrm{SiO}}$ : Surfactant is added to toluene (5 wt. \%) and stirred by hand shaking to form the inverse micelle solution. $\mathrm{PtCl}_{2}(0.005 \mathrm{M})$ and the gel precursor TEOS $(0.4 \mathrm{M})$ are added to the DDAB, toluene mixture, and the solution is stirred until the salt is fully solubilized. Sufficient $\mathrm{LiBH}_{4} / \mathrm{THF}$ solution to result in a 3:1 ratio of $\mathrm{BH}_{4}^{-}: \mathrm{Pt}^{2+}$ is then injected into the salt precursor solution under rapid stirring to form the Pt clusters. The $40 \mathrm{wt}$ \% $\%$ TBAOH in water solution is added to the mixture 5 minutes after the addition of the $\mathrm{LiBH}_{4}$, and gelation is marked when the solution no longer flows under gravity. The water to gel precursor molar ratio is set at 3:1. The gels are aged 
for two days at $50^{\circ} \mathrm{C}$ and are washed with pure toluene (soaked for several hours at $50^{\circ} \mathrm{C}, 3$ cycles) to remove any unattached surfactant, metal salt, or excess reducing agent. The samples are then split in two with half being used to form xerogels and the other half being used to form aerogels. Xerogels are formed by three staged drying cycles of $40^{\circ} \mathrm{C}, 50^{\circ} \mathrm{C}$, and $120^{\circ} \mathrm{C}$ each overnight. Aerogels are formed under supercritical $\mathrm{CO}_{2}$ extraction. The overall molar equivalent ratio of the synthesis is $\mathrm{Si}: 3 \mathrm{H}_{2} \mathrm{O}: 23$ toluene : $0.2 \mathrm{TBAOH}$ where the toluene actually represents $0.005 \mathrm{M} \mathrm{PtCl}_{2}$ in $5 \mathrm{wt}$. \% $\mathrm{DDAB}$ in toluene.

Sequential inverse micelle, sol-gel derived $P t / \mathrm{Al}_{2} \mathrm{O}_{3}$ : The $\mathrm{Pt} / \mathrm{Al}_{2} \mathrm{O}_{3}$ synthesis is loosely based on a previous alcohol based $\mathrm{Al}_{2} \mathrm{O}_{3}$ recipe without metal (33). DDAB is added to toluene ( $5 \mathrm{wt}$. \%) and stirred by hand shaking to form the inverse micelle solution. The gel precursor is a 1:1 molar ratio of aluminum tri-sec-butoxide and ethyl acetoacetate. $\mathrm{PtCl}_{2}$ $(0.005 \mathrm{M})$ and the gel precursor $(0.8 \mathrm{M})$ are added to the DDAB, toluene mixture, and the solution is stirred until the salt is fully solubilized. The $\mathrm{LiBH}_{4} / \mathrm{THF}$ solution is then injected into the salt precursor solution to form the clusters under rapid stirring so that the $\left[\mathrm{BH}_{4}^{-}\right]$: $\left[\mathrm{Pt}^{2+}\right]=3: 1$. After 30 minutes, an aqueous $4.3 \mathrm{M} \mathrm{HNO}_{3}$ solution is added, and gelation is marked when the solution no longer flows under gravity. The water to gel precursor molar ratio is set at $3: 1$. The gels are aged for two days at $50^{\circ} \mathrm{C}$ and are washed with pure toluene (soaked for several hours at $50^{\circ} \mathrm{C}, 3$ cycles) to remove any unattached surfactant, metal salt, or excess reducing agent. The samples are then split in two with half being used to form xerogels and the other half being used to form aerogels. Xerogels are formed by three staged drying cycles of $40^{\circ} \mathrm{C}, 50^{\circ} \mathrm{C}$, and $120^{\circ} \mathrm{C}$ each overnight. Aerogels are formed under supercritical $\mathrm{CO}_{2}$ extraction. The overall molar equivalent ratio of the synthesis is $\mathrm{Al}: 3 \mathrm{H}_{2} \mathrm{O}$ : 14 toluene : $0.2 \mathrm{HNO}_{3}$ where the toluene represents $0.005 \mathrm{M} \mathrm{PtCl}_{2}$ in 5 wt. \% DDAB in toluene.

Concurrent inverse micelle, sol-gel derived $\mathrm{Pt} / \mathrm{SiO} \mathrm{O}_{2}:$ An asterisk denotes samples made by the 'concurrent' methodology. Here, Pt clusters are synthesized with a 'standardized' inverse micelle recipe. Concurrently, $\mathrm{SiO}_{2}$ monolith is synthesized with a 'standardized' ethanol sol-gel recipe. The clusters are then allowed to diffuse into the gel, and the gel is dried leading to encapsulation of the particles in the micropores. To form the clusters, $0.01 \mathrm{M} \mathrm{PtCl}_{2}$ is solubilized in a $5 \mathrm{wt}$. \% DDAB, toluene mixture. The $\mathrm{LiBH}_{4} / \mathrm{THF}^{2}$ solution is injected into the salt precursor solution under rapid stirring so that the $\left[\mathrm{BH}_{4}^{-}\right]$: 
$\left[\mathrm{Pt}^{2+}\right]$ ratio is 3:1. To form the $\mathrm{SiO}_{2}$ monolith, a stock solution of $61 \mathrm{ml} \mathrm{TEOS}, 61 \mathrm{ml}$ ethanol, $4.87 \mathrm{ml}$ water, and $0.2 \mathrm{ml} 1 \mathrm{M} \mathrm{HCl}$ is refluxed at $60^{\circ} \mathrm{C}$ for $1.5 \mathrm{hrs}$ with stirring. The solution is stable indefinitely at $20^{\circ} \mathrm{C}$, but is stored in a freezer to ensure stability. The approximate molar ratio of the solution is TEOS : 4 ethanol : $\mathrm{H}_{2} \mathrm{O}: 0.007 \mathrm{HCl}$. Mixing a 10:1 volume ratio of stock solution to $0.5 \mathrm{M} \mathrm{NH}_{4} \mathrm{OH}$ causes gelation within minutes. The gels are aged and washed three times with excess ethanol at $50^{\circ} \mathrm{C}$ for several hours. To produce the cluster / $\mathrm{SiO}_{2}$ matrix, the gels are washed three times with excess toluene at $50^{\circ} \mathrm{C}$ for several hours. Then, the $\mathrm{SiO}_{2}$ is washed with excess cluster solution three times at $50^{\circ} \mathrm{C}$ for several hours. Xerogels are formed by three staged drying cycles of $40^{\circ} \mathrm{C}, 50^{\circ} \mathrm{C}$, and $120^{\circ} \mathrm{C}$ each overnight. The methodology was not extended to aerogels or $\mathrm{Al}_{2} \mathrm{O}_{3}$ supports, although this should be facile.

All samples were calcined in a tube furnace under air. A two stage temperature ramp was used. The samples were held at $250^{\circ} \mathrm{C}$ for 1 hour and then the final temperature for 1 hour. Final calcine temperatures were determined by TGA results as discussed below.

Characterization. Metal weight fractions on the supports were determined with a PerkinElmer 5100PC atomic absorption spectrophotometer (AA). Thermal gravimetric analysis (TGA) was completed with a TA Instruments Thermal Analyst 2000. Platinum particle size was characterized with transmission electron microscopy (TEM) with a $300 \mathrm{keV}$ Phillips CM30 electron microscope. To prepare TEM samples, dried, crushed gels were dispersed over a holey carbon substrate. $\mathrm{N}_{2}$ porosimetry, surface area analysis (BET) of the crushed xerogels and aerogels was carried out with a Quantachrome Autosorb- 6 surface analysis apparatus. BET samples were outgassed for 20 hours at $100^{\circ} \mathrm{C}$. Hydrogen chemisorption was measured using a Coulter Omnisorp $100 \mathrm{CX}$ instrument. All chemisorption samples were calcined at $450^{\circ} \mathrm{C}$ in air for 1 hour except the 'concurrently' produced $\mathrm{Pt} / \mathrm{SiO}_{2}$ ' sample. Based on TGA results, the concurrently produced $\mathrm{Pt}_{/} \mathrm{SiO}_{2}^{*}$ sample was calcined at $600^{\circ} \mathrm{C}$ for 1 hour in air. In the chemisorption unit, the samples were outgassed in flowing $\mathrm{He}$ at $350^{\circ} \mathrm{C}$ for 1 hour followed by reduction in flowing hydrogen at $400^{\circ} \mathrm{C}$ for 2 hours. Samples were then evacuated at $405^{\circ} \mathrm{C}$ for 3 hours prior to cooling to room temperature before measurement of hydrogen uptakes. Dispersions were calculated from total hydrogen uptake and converted to particle sizes by assuming spherical particles and a Pt atom surface density of $1.25 \times 10^{15}$ atoms $/ \mathrm{cm}^{2}$. The samples used for TEM, chemisorption, and propane 
dehydrogenation are from the set of samples described by the first column of the AA results (i.e., those listed first and on the left in Table 3).

Catalyst Testing. All samples were initially in the form of a single monolithic piece. These monoliths were carefully ground and sieved to obtain a $-60 /+80$ mesh fraction for use in reactor testing. The remainder of the sample disintegrated into a fine powder which was used for the majority of the physical characterization. The 'sequentially' produced $\mathrm{Pt} / \mathrm{SiO}_{2}$ sample was tested for propane dehydrogenation in the powdered state since careful grinding of the monolith did not result in a sufficient quantity of $-60 /+80$ mesh material.

Activity measurements were made in a fixed bed flow reactor. The reaction sequence begins with heating of the catalyst bed to reaction temperature (typically $525-575^{\circ} \mathrm{C}$ ) in flowing nitrogen. Once temperature is reached, the nitrogen flow is stopped and reactant flow begins. Propane is fed through a calibrated mass flow controller and is preheated in a $1 / 8$ " coiled stainless steel tube at the reactor inlet. The preheated reactant then enters a $3 / 8$ " stainless steel tube containing catalyst. The catalyst is supported on a fine steel mesh located at the bottom of the reactor. Depending on catalyst density, 0.3-0.6 gm of catalyst are typically used to give a catalyst bed volume of $3.6 \mathrm{cc}$. After exiting the reactor, product gases are exhausted through the sample loop of a gas chromatograph, where C1-C4 hydrocarbons are analyzed. Gas sampling and analysis was performed at 20 minute intervals. Oxygen regeneration and hydrogen reduction treatments were often used alternately to achieve maximum catalyst activity. Reactor temperature is measured by three thermocouples externally attached to the top, middle, and bottom of the reactor, and one thermocouple located inside the reactor at the bottom of the catalyst bed. No significant differences were ever observed among the four thermocouples indicating that the reactor was operating in an isothermal mode.

In general, no consistent activation behavior was observed among the various catalysts. In most cases, hydrogen reduction was found to be beneficial, but for one catalyst $\left(\mathrm{Pt} / \mathrm{Al}_{2} \mathrm{O}_{3}\right.$ aerogel) hydrogen reduction actually decreased activity. Also, the rate of deactivation of the catalysts varied, as did the efficacy of air oxidation to burn off carbon and regenerate the catalysts. Because of these variations among the catalysts, quantitative comparisons are difficult. The reported activities are therefore the best activities that were achieved on each catalyst, and the prior history of the catalysts varies substantially. In addition to variations in pretreatment, variations in both weight hourly space velocity 
(WHSV) and gas hourly space velocity (GHSV) also occurred among the catalysts. The GHSV variations arise as a result of a loss of catalyst bed volume during the reactions, which prevents an a priori prediction of the true GHSV. The WHSV velocities result from both the bed volume changes, as well as differences in density among the catalysts. In general, the initial bed volume was kept constant at $\sim 3.6 \mathrm{cc}$, provided a sufficient quantity of the $-60 /+80$ mesh fraction was available. As a result, variations in WHSV are much greater than variations in GHSV. In order to compensate for the space velocity variations, both propane conversion/selectivity and propane turnover frequencies (TOF, calculated from measured dispersions, WHSV's and Pt loadings) are reported. The conversion/selectivity data allow comparison with a commercial $7.5 \mathrm{wt} \% \mathrm{Cr} / \mathrm{Al}_{2} \mathrm{O}_{3}$ catalyst (Girdler G-41), while the TOFs allow comparisons of intrinsic activity among the catalysts. Because conversions are generally less than $10 \%$ and therefore far from equilibrium (equilibrium conversion is $\sim 30 \%$ at $550^{\circ} \mathrm{C}$ ), the use of TOFs should compensate for space velocity differences and provide a valid measure of intrinsic activity.

\section{Results}

TGA weight loss results indicate a large difference between the samples produced by the sequential reactions of metal reduction, hydrolysis and condensation and the $\mathrm{SiO}_{2}{ }^{*}$ sample produced by the concurrent synthesis (Figure 2). The weight loss is large on an absolute scale for the samples produced in the sequential methodology with greater than $50 \%$ weight loss. Most weight loss occurs below $400^{\circ} \mathrm{C}$ so $450^{\circ} \mathrm{C}$ was chosen as the calcination temperature. The $\mathrm{Pt} / \mathrm{SiO}_{2}{ }^{*}$ sample shows two weight loss regions, at approximately $350^{\circ} \mathrm{C}$ and $550^{\circ} \mathrm{C}$. Only $15 \mathrm{wt} . \%$ of the total sample is lost. $600^{\circ} \mathrm{C}$ was therefore chosen as the calcination temperature to ensure complete weight loss prior to analysis and testing. TGA results did not differ in air compared to an inert He atmosphere.

BET surface area results indicate a large difference between the samples produced by the sequential reactions and the $\mathrm{SiO}_{2}^{*}$ sample produced by the concurrent synthesis (Figure 3). 'Sequentially' produced $\mathrm{Pt} / \mathrm{SiO}_{2}$ and $\mathrm{Pt}_{2} \mathrm{Al}_{2} \mathrm{O}_{3}$ xerogels and aerogels are nonporous and show little surface area. Upon calcination surface area increases greatly but then decreases during propane dehydrogenation. The same trend is observed with the $\mathrm{Pt} / \mathrm{SiO}_{2}{ }^{*}$ xerogel, but these samples are mesoporous with high surface areas even prior to calcining. Differential 
pore size analysis shows the $\mathrm{Pt} / \mathrm{SiO}_{2}{ }^{*}$ xerogel pores are larger with a broader pore size distribution then the other samples (Figure 4).

Particle size was studied with TEM (Figure 5) and $\mathrm{H}_{2}$ chemisorption. TEM analysis indicates the initial $\mathrm{Pt} / \mathrm{SiO}_{2}$ xerogel, $\mathrm{Pt} / \mathrm{SiO}_{2}$ aerogel, and the $\mathrm{Pt} / \mathrm{SiO}_{2}{ }^{*}$ xerogel particle diameters are $1.5 \mathrm{~nm}, 1.5 \mathrm{~nm}$, and $2.0 \mathrm{~nm}$, respectively. After calcination, TEM particle sizes

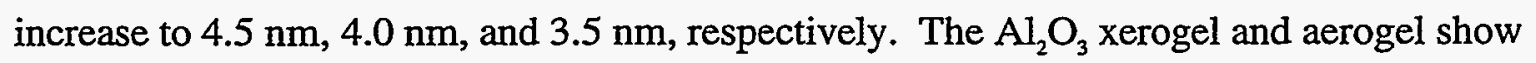
aggregated metal particles throughout the substrate. No individual particles are observed. After calcination, individual particles are observed. The $\mathrm{Pt} / \mathrm{Al}_{2} \mathrm{O}_{3}$ xerogel and aerogel particle diameters are $3.5 \mathrm{~nm}$ and $>50 \mathrm{~nm}$, respectively. Particle sizes determined by chemisorption are compared to TEM results (Table2). For the calcined $\mathrm{Pt} / \mathrm{SiO}_{2}$ and $\mathrm{Pt} / \mathrm{SiO}_{2}{ }^{\circ}$ aerogels and the calcined $\mathrm{P} / \mathrm{Al}_{2} \mathrm{O}_{3}$ xerogel, the measured dispersions are $12.7 \%, 22.3 \%$, and $11.1 \%$, respectively, corresponding to particle diameters of $4.5,2.5$, and $4.9 \mathrm{~nm}$, in close agreement with TEM results. $\mathrm{H}_{2}$ chemisorption measurements were not possible on the uncalcined samples due to severe outgassing problems.

Atomic absorption spectroscopy was used to determine metal loadings. Three separate sample sets were tested (Table 3). The first set of numbers (on the left) represent the samples used for BET, TEM, chemisorption, and activity testing. For the $\mathrm{Pt} / \mathrm{SiO}_{2}$ xerogel, $\mathrm{Pt} / \mathrm{SiO}_{2}$ aerogel, $\mathrm{Pt} / \mathrm{SiO}_{2}{ }^{*}$ xerogel, $\mathrm{Pt} / \mathrm{Al}_{2} \mathrm{O}_{3}$ xerogel and $\mathrm{Pt} / \mathrm{Al}_{2} \mathrm{O}_{3}$ aerogel, the metal loadings are $0.24+/-0.08$ wt. $\%, 0.23+/-0.07$ wt. $\%, 0.25+/-0.03$ wt. $\%, 0.22+/-0.14$ wt. $\%$, and $0.24+/-0.14$ wt. \%, respectively. Reproducibility is poor, but it is particularly poor with the $\mathrm{Al}_{2} \mathrm{O}_{3}$ samples indicating an extreme sensitivity of metal loading to synthesis conditions. It is possible to increase metal loadings by increasing precursor salt concentrations, but particle size and polydispersity generally increases.

The platinum samples were tested for propane dehydrogenation at $550^{\circ} \mathrm{C}$ (Table 4 , Figure 6). Note the WHSV's and pretreatment conditions differ significantly among the six samples studied, as discussed earlier. Because of these variations, quantitative activity comparisons cannot be made, and the activity measurements serve mainly to demonstrate the potential of this class of dehydrogenation catalysts, as will be discussed later.

\section{Discussion}

Synthesis Effects on Support Properties. TGA results indicate that for samples

synthesized via the sequential methodology (i.e., sol-gel processing is completed in an 
alcohol free, cluster, inverse micelle system) over $50 \mathrm{wt}$. \% of the sample is thermally lost by about $400^{\circ} \mathrm{C}$. The only single component present to such extent in the initial reaction mixture is the gel precursor TEOS leading us to believe that the large weight loss in the final product is due to unreacted TEOS monomer. It is likely that the unreacted TEOS results from inefficient hydrolysis and condensation reactions in apolar, alcohol free inverse micelle mixtures. The same argument holds for the alumina system.

In samples synthesized via the concurrent methodology (i.e., using a well defined solgel process taking place in ethanol followed by the addition of an already prepared cluster, inverse micelle solution), TGA results show two weight loss regions with a total loss of approximately $12 \mathrm{wt} . \%$. No weight loss occurs before $200^{\circ} \mathrm{C}$ indicating all solvents were removed in the drying procedure. At approximately $200^{\circ} \mathrm{C}$, there is a loss of about $6 \mathrm{wt}$. \% mostly due to the thermal breakdown of surfactant. There is little unreacted TEOS due to the highly studied, efficient synthesis procedure used. Finally, starting at approximately $400^{\circ} \mathrm{C}$, about $5 \mathrm{wt}$. \% is lost probably due to the thermal breakdown of terminal hydroxy groups.

The $\mathrm{N}_{2}$ porosimetry results support our TGA analysis. In sequentially synthesized samples, no measurable surface area exists before calcination, because of the presence of surfactant and unreacted TEOS in the pores. The surface area increases greatly upon thermal removal of these components. In the concurrently synthesized sample, surface area increases slightly due to removal of surfactant.

In our earlier work, we studied the synthesis parameters that govern final material properties in sol-gel processed materials in inverse micelle systems (21). We observed, as we observe here, that monoliths form at much lower water:TEOS ratios than in alcohol based systems. We speculate that monoliths form due to localized high water concentration regions in the inverse micelles with the hydrolysis and condensation reaction mechanisms taking place across surfactant monolayers and in transient micelle material exchange states. It appears in these mechanisms that only a fraction of TEOS is exposed to the water rich regions and participates in the reaction, thus providing a high effective water concentration as we speculated earlier. Also in our previous work, we noticed a reverse trend in surface area with the water:TEOS ratio than is typically found in alcohol systems. As the samples in our previous study were not calcined, it is unfortunate that we were measuring surface areas of materials obstructed with unreacted TEOS. True monolithic surfaces were not measured calling into question the analysis of the effect of the water:TEOS ratio in that work. 
Synthesis Effects on Particle Properties. TEM results indicate that the metal reduction reaction to form clusters is highly efficient in the $\mathrm{SiO}_{2}$ samples. Small, monodisperse particles with diameters of less than $2 \mathrm{~nm}$ is typical for Pt particles synthesized in pure DDAB/toluene inverse micelle mixtures. Visually, an abrupt color change and rapid $\mathrm{H}_{2}$ gas production indicate an instantaneous reaction. In the sequential reduction methodology, the presence of TEOS, the hydrolysis and condensation reactions, and drying have no effect on final particle size. In the concurrent reduction methodology, diffusion of the particles into the gel network and drying have no effect on final particle size.

The reduction reaction efficiency decreases substantially in alumina systems.

Visually, color change and $\mathrm{H}_{2}$ production occurs over at least one-half hour. In reactions in inverse micelles, it is known that slow nucleation and growth results in large, polydisperse size distributions. We observe similar results here. Further, the particles are aggregated indicating surfactant stabilization was not effective. A concurrent reaction mechanism where the particles are synthesized separately maybe required to obtain highly dispersed $\mathrm{Pt}$ particles in aluminum gels. We have not yet studied a concurrent reaction mechanism for alumina.

\section{Sintering and the Relationship between Particle and Pore Sizes. In the sequentially}

synthesized $\mathrm{SiO}_{2}$ samples, particle size and pore dimensions are relatively equal before calcination. After calcination, the particle size increases to approximately three times larger than the pore dimensions even at the relatively low calcination temperatures of $450^{\circ} \mathrm{C}$. Agreement between TEM and chemisorption results indicate the particles are moving out of the pores and unto the surface of the substrates rather than being occluded in the support structure.

In the concurrently synthesized $\mathrm{Pt} / \mathrm{SiO}_{2}{ }^{*}$ xerogel, particle size is approximately three times smaller than the pore dimension before calcination. After calcination, the particle size and the pore dimensions grow only slightly (i.e. no sintering), the particle size remains approximately three times smaller than the pore dimension. Agreement between TEM and chemisorption results indicate no particle occlusion.

It is clear from the above results that no clear relationship between particle size and pore dimension effects sintering behavior. Previous work on $\mathrm{Pt} / \mathrm{SiO}_{2}$ catalysts (18) states that there is a strong relationship between particle size and pore size, and that for low metal loadings sintering is prevented when particle sizes match average poor diameters. The conclusions of this previous work were based upon three distinct cases: 1) 'high' Pt loadings 
with particle sizes smaller than the pore size; 2) 'high' Pt loadings with particle sizes matched to the pore size; and 3) 'low'Pt loadings with particle sizes matched to the pore size. The fourth case, 'low' Pt loadings with particle sizes smaller than the pore size, was not studied. Thus, the possibility that sintering resistance is merely a reflection of Pt loading only could not be eliminated. The concurrently synthesized $\mathrm{Pt} / \mathrm{SiO}_{2}{ }^{*}$ xerogel reported here falls into the fourth case, and demonstrates resistance to sintering even though the particle size and pore size are not well matched.

The results from the previous work (18) combined with our results for the $\mathrm{Pt} / \mathrm{SiO}_{2}{ }^{*}$ sample is further evidence that there is no clear relationship between particle size and pore dimension effecting sintering behavior. The combined results with $\mathrm{Pt}_{\mathrm{SiO}}{ }_{2}^{*}$ provide strong evidence that sintering is a function of metal loading. Metal particle sintering on support materials occurs by two mechanisms: (1) particle aggregation due to particle diffusion along the support, and (2) Ostwald ripening where large particles grow at the expense of smaller particles due to atomization and diffusion between particles. As Ostwald ripening is concentration dependent, it appears Ostwald ripening is the predominant mechanism for sintering. It may be possible to effect vapor pressures and influence surface tensions by encapsulation such that the mechanism for Ostwald ripening is hindered, but there is yet no experimental evidence to quantify the effects. The relationship between the initial particle size and pore dimension may effect particle to particle diffusion, thus somewhat preventing the less important form of sintering. Paradoxically, however, sintering is observed in the $\mathrm{Pt} / \mathrm{SiO}_{2}$ xerogel where metal loading is low (while the average metal loading for the $\mathrm{Pt} / \mathrm{SiO}$ xerogel is relatively high recall the sample tested in BET, TEM, chemisorption, and catalyst testing is the one reported in the left most column of Table Im). It appears that sintering is more complicated that concentration dependent Ostwald ripening also.

The effect of calcination on particle size in alumina samples is counter intuitive. Encapsulation is not an issue, because the particles and aggregates are much larger than the pore dimensions. With calcination, aggregated particles separate into smaller domains. In alumina samples, random particle diffusion upon calcination apparently decreases the particle concentration gradient resulting in more dispersed, smaller particles.

Catalyst Testing. The propane conversion data in Table 4 shows that the uncalcined $\mathrm{Pt}_{\mathrm{SiO}}{ }_{2}^{*}$ xerogel results in the highest conversions of any of the Pt catalysts. Note, however, that the space velocities for this catalyst are substantially lower than for the other catalysts. 
Of the other catalysts, the calcined $\mathrm{Pt} / \mathrm{SiO}_{2}{ }^{*}$ xerogel gives the highest conversions, although the performance of the $\mathrm{Pt} / \mathrm{Al}_{2} \mathrm{O}_{3}$ aerogel is comparable if a linear correction for the difference in GHSV is made. Even with corrections for GHSV differences, none of the catalysts performed comparably to the commercial Cr catalyst, which achieved near equilibrium conversion with a very high selectivity. In general, the selectivities of the $\mathrm{Pt}$ catalysts are inferior to the commercial catalyst, particularly at the higher conversions. The selectivities are, however, substantially better than those reported by de Miguel, et al. (27) at $853 \mathrm{~K}$, and comparable to those reported by Bariås, et al. (25) at $519^{\circ}$

On a TOF basis, the calcined $\mathrm{Pt} / \mathrm{SiO}_{2}{ }^{*}$ xerogel and the $\mathrm{Pt} / \mathrm{Al}_{2} \mathrm{O}_{3}$ aerogel have by far the highest activities. No calculations of TOFs are possible for the commercial catalyst or the uncalcined $\mathrm{Pt} / \mathrm{SiO}_{2}{ }^{*}$ xerogel due to the absence of an appropriate active site counting method for the former, and severe outgassing problems for the latter, which prevent hydrogen uptake measurements. The magnitudes of the TOFs on the two best catalysts are substantially higher than those reported by Bariås, et al. (25) for traditionally prepared $\mathrm{Pt} / \mathrm{SiO}_{2}$ or $\mathrm{Pt} / \mathrm{Al}_{2} \mathrm{O}_{3}$ catalysts at $519 \mathrm{~K}$. Furthermore, while Bariås, et al. noted rapid deactivation over a period of -30 minutes, resulting in loss of more than $90 \%$ of the initial activity, we find less than a $50 \%$ loss in activity for the $\mathrm{Pt} / \mathrm{Al}_{2} \mathrm{O}_{3}$ aerogel, and only a $10 \%$ loss for the $\mathrm{Pt} / \mathrm{SiO}_{2}{ }^{*}$ xerogel catalyst over a similar time period. In fact with the exception of the $\mathrm{Pt} / \mathrm{SiO}_{2}$ aerogel, all of our catalysts show a remarkable resistance to deactivation (Table 2). This resistance is comparable to that reported by Bariås, et al. (25) for traditionally prepared $\mathrm{PtSn} / \mathrm{Al}_{2} \mathrm{O}_{3}$ and $\mathrm{PtSn} / \mathrm{SiO}_{2}$ catalysts. Furthermore, the initial activities of our two best catalysts are comparable to those of the $\mathrm{PtSn} / \mathrm{Al}_{2} \mathrm{O}_{3}$ and $\mathrm{PtSn} / \mathrm{SiO}_{2}$ catalysts of Bariås, et al. Thus, through the use of sol-gel techniques to prepare Pt clusters inside $\mathrm{SiO}_{2}$ and $\mathrm{Al}_{2} \mathrm{O}_{3}$ xerogels and aerogels, we have obtained performance that is comparable to $\mathrm{PtSn} / \mathrm{Al}_{2} \mathrm{O}_{3}$ and $\mathrm{PtSn} / \mathrm{SiO}_{2}$ catalysts prepared by incipient wetness. Since deactivation in these catalysts is traditionally attributed to coke formation and the addition of $\mathrm{Sn}$ is known to inhibit coke formation $(25,27)$, it is probable that the sol-gel derived catalysts also somehow inhibit coke formation, although as yet we have no physical evidence to support this hypothesis. This conclusion is consistent with previous work (20).

\section{Conclusions}


We introduce two novel synthesis strategies to make nanoclusters on silica and alumina supports using inverse micelle technology and sol-gel processing. In the first methodology, sol-gel chemistry is performed in alcohol-free inverse micelle, cluster solutions. Hydrolysis and condensation reactions are inefficient, but nonetheless monoliths form around the clusters. In the second method, clusters formed in traditional inverse micelle solutions are allowed to diffuse into pre-existing monoliths formed using traditional sol-gel techniques (hydrolysis and condensation in ethanol). The two techniques give materials with similar cluster sizes, but different pore dimensions. We conclude that the relative sizes of particles and pores has no clear effect on sintering behavior. Sintering appears to be predominately dependent on metal loading suggesting Ostwald ripening as the sintering: mechanism, but even this analysis is oversimplified. Our samples achieved relatively high catalytic activity and stability over time for propane dehydrogenation indicating that the synthesis methods described here result in catalyst performance more typical of $\mathrm{PtSn} / \mathrm{Al}_{2} \mathrm{O}_{3}$ or $\mathrm{PtSn} / \mathrm{SiO}_{2}$ catalysts prepared by impregnation. This behavior may indicate a high resistance to coke formation in our catalysts. 


\section{References}

1. Brinker, C.J., and Scherer, G.W., "Sol-Gel Science," Acedemic Press Inc., 1990.

2. Ward, D.A., and Ko, E.I., I\&EC Research 34, 421 (1995).

3. Schneider, M., and Baiker, A., Catal. Rev.-Sci. Eng. 37, 515 (1995).

4. Cauqui, M.A., and Rodriquez-Izquierdo, J.M., J. of Non-Cryst. Solids 147\&148, 724 (1992).

5. Pajonk, G.M., Appl. Catal. 72, 217 (1991).

6. Tominaga, H., Ono, Y., and Keii, T., J. Catal. 40, 197 (1975).

7. Ikoma, S., Takano, S., Nomoto, E., and Yokoi, H., J. Non-Cryst. Solids 113, 130 (1989).

8. Morke, W., Lamber, R., Schubert, U., and Breitscheidel, B., Chem. Mater. 6, 1659 (1994).

9. Ishiyama, J.I., Kurokawa, Y., Nakayama, T., and Imaizumi, S., Appl. Catal. 40, 139 (1988).

10. Lopez, T., Villa, M., and Gomez, R., J. Phys. Chem. 95, 1690 (1991).

11. Lopez, T., Lopez-Gaona, A., and Gomez, R., Langmuir 6, 1343 (1990).

12. Moon Choi, K., and Shea, K.J., J. Am. Chem. Soc. 116, 9052 (1994).

13. Hardee, J.R., Tunney, S.E., Frye, J., and Stille, J.K., J. Polym. Sci., Part A: Polym. Chem. 28, 3669 (1990).

14. Azomoza, M., Lopez, T., Gomez, R., and Gonzalez, R.D., Catal. Today 15, 547 (1992).

15. Lopez, T., Herrera, L., Gomez, R., Zou, W., Robinson, K., and Gonzalez, R.D., J. Catal. 136, 621 (1992).

16. Gomez, R., Lopez, T., Castillo, S., and Gonzalez, R.D., J. Sol-Gel Sci. Technol. 1, 205 (1994).

17. Lopez, T., Bosch, P., Navarrete, J., Azomoza, M., and Gomez, R., J. Sol-Gel Sci. Technol. 1, 193 (1994).

18. Zou, W., and Gonzalez, R.D., Appl. Catal. A 102, 181 (1993).

19. Mizushima, Y., and Hori, M., Appl. Catal. A 88, 137 (1992).

20. Balakrishnan, K., and Gonzalez, R.D., J.Catal. 144, 395 (1993).

21. Martino A., Yamanaka, S.A., Kawola, J.S., and Loy D.A., Chem. Mater. 9, 423 (1997).

22. Bradley, J.S., "Clusters and Colloids, From Theory to Applications," Schmid, G., Ed., VCH Publications Inc., 1994, Ch. 6.

23. Steigerwald, M.L., and Brus, L.E., Annu. Rev. Mater. Sci. 19, 471 (1989).

24. Lipinsky, E.S., and Ingham, J.D., "Brief Characterization of the Top 50 U.S. Commodity Chemicals," Pacific Northwest National Laboratory task report, Hanford, WA, 1994, pp. 26-27, 40-41.

25. Barias, O.A., Holmen, A., and Blekkan E.A., J. Catal. 158, 1 (1996).

26. Cortright, R.D., and Dumesic, J.A., J. Catal. 157, 576 (1995).

27. de Miguel, S., Castro, A., Scelza O., Garcia Fierr, J.L., and Soria, J., Catalysis Lett. 36, 201 (1996).

28. Stagg, S.M., Querini, C.A., Alvarez, W.E., and Resasco, D.E., J. Catal. 168, 75 (1997).

29. Cavani, F., Koutyrez, M., Trifiro, F. Bartolini, A., Ghisletti, D., Iezzi, R., Santucci, A., and Del Picro, G., J. Catal. 158, 236 (1996).

30. Loc, L.C., Gaidai, N.A., Kiperman, S.L., and Thoang, H.S., Kinetics and Catalysis 36, 511 (1995). 
31. Udomsak, S., and Anthony, R.G., Ind. Eng. Chem. Res. 35, 47 (1996).

32. Pena, J.A., Monzou, A., and Santamaria, J., J. Catal. 142, 59 (1993).

33. Bonhomme-Coury, L., Babonneau, F., Livage, J., J. Sol-Gel Sci. Technol. 3, 157 (1994). 
Table 1. Summary of samples, recipes, and post-synthesis treatments used in this study. The asterisk always refers to the sample synthesized by the 'concurrent' procedure explained in the text.

\begin{tabular}{|c|c|c|}
\hline Catalyst & Recipe & $\begin{array}{l}\text { Post-Synthesis } \\
\text { Treatment }\end{array}$ \\
\hline $\mathrm{Pt} \mathrm{SiO}_{2}$ xerogel & $\begin{array}{l}\mathrm{Si} \cdot 3 \mathrm{H}_{2} \mathrm{O} \cdot 23 \mathrm{DDAB} / \mathrm{Tol} \cdot 0.2 \mathrm{TBAOH} \bullet \\
0.005 \mathrm{M} \mathrm{PtCl}_{2}\end{array}$ & $\begin{array}{l}\text { washed; } 40,50, \\
120^{\circ} \mathrm{C} \text { air drying } \\
\text { stages }\end{array}$ \\
\hline $\mathrm{Pt} / \mathrm{SiO}_{2}$ aerogel & $\begin{array}{l}\mathrm{Si} \cdot 3 \mathrm{H}_{2} \mathrm{O} \cdot 23 \mathrm{DDAB} / \mathrm{Tol} \cdot 0.2 \mathrm{TBAOH} \bullet \\
0.005 \mathrm{M} \mathrm{PtCl}_{2}\end{array}$ & $\begin{array}{l}\text { Supercritical } \\
\text { extraction: } \mathrm{CO}_{2}, 40^{\circ} \mathrm{C}\end{array}$ \\
\hline $\mathrm{Pt} / \mathrm{Al}_{2} \mathrm{O}_{3}$ xerogel & $\begin{array}{l}\mathrm{Al} \cdot 3 \mathrm{H}_{2} \mathrm{O} \cdot 14 \mathrm{DDAB} / \mathrm{Tol} \cdot 0.2 \mathrm{HNO}_{3} \bullet \\
0.005 \mathrm{M} \mathrm{PtCl}_{2}\end{array}$ & $\begin{array}{l}\text { washed; } 40,50, \\
120^{\circ} \mathrm{C} \text { air drying } \\
\text { stages }\end{array}$ \\
\hline $\mathrm{Pt} / \mathrm{Al}_{2} \mathrm{O}_{3}$ aerogel & $\begin{array}{l}\mathrm{Al} \cdot 3 \mathrm{H}_{2} \mathrm{O} \cdot 14 \mathrm{DDAB} / \mathrm{Tol} \cdot 0.2 \mathrm{HNO}_{3} \bullet \\
0.005 \mathrm{M} \mathrm{PtCl}_{2}\end{array}$ & $\begin{array}{l}\text { Supercritical } \\
\text { extraction: } \mathrm{CO}_{2}, 40^{\circ} \mathrm{C}\end{array}$ \\
\hline $\mathrm{Pt} / \mathrm{SiO}_{2} *$ xerogel & $\begin{array}{l}\mathrm{Si} \cdot 4 \mathrm{EtOH} \cdot \mathrm{H}_{2} \mathrm{O} \cdot 0.007 \mathrm{HCl} \\
0.1 \mathrm{H}_{2} \mathrm{O} \cdot 0.05 \mathrm{M} \mathrm{NH}_{4} \mathrm{OH} ; 0.01 \mathrm{M} \mathrm{PtCl}_{2}\end{array}$ & $\begin{array}{l}\text { washed; } 40,50, \\
120^{\circ} \mathrm{C} \text { air drying } \\
\text { stages }\end{array}$ \\
\hline
\end{tabular}


Table 2. Summary of particle diameters as determined by TEM. TEM results are compared to chemisorption results for the post-calcined samples.

\begin{tabular}{lccc}
\hline & \multicolumn{3}{c}{ Particle Diameter (nm) } \\
\cline { 2 - 4 } Catalyst & Post-Synthesis & \multicolumn{2}{c}{ Post-Calcination } \\
\cline { 2 - 4 } & TEM & 4.5 & Chemisorption \\
\hline $\mathrm{Pt} / \mathrm{SiO}_{2}$ xerogel & 1.5 & & 4.5 \\
\hline $\mathrm{Pt} / \mathrm{SiO}_{2}$ aerogel & 1.5 & 4.0 & 4.9 \\
\hline $\mathrm{Pt} / \mathrm{Al}_{2} \mathrm{O}_{3}$ xerogel & aggregated & 3.5 & \\
\hline $\mathrm{Pt} / \mathrm{Al}_{2} \mathrm{O}_{3}$ aerogel & aggregated & $>50$ & 2.5 \\
\hline $\mathrm{Pt} / \mathrm{SiO}_{2}{ }^{*}$ xerogel & 2.0 & 3.5 & \\
\hline
\end{tabular}

Table 3. Summary of weight percent metal loading as determined by atomic absorption (AA). Multiple measurements show the reproducibility of the synthesis.

\begin{tabular}{lcc}
\hline \multirow{2}{*}{ Catalyst } & \multicolumn{2}{c}{ Metal Loading (wt. \%) } \\
\cline { 2 - 3 } & uncalcined & calcined \\
\hline $\mathrm{Pt} / \mathrm{SiO}_{2}$ xerogel & $0.16,0.25,0.31$ & $0.32,0.50,0.62$ \\
\hline $\mathrm{Pt} / \mathrm{SiO}_{2}$ aerogel & $0.15,0.27,0.26$ & $0.43,0.77,0.74$ \\
\hline $\mathrm{Pt} / \mathrm{Al}_{2} \mathrm{O}_{3}$ xerogel & $0.20,0.10,0.37$ & $0.50,0.25,0.92$ \\
\hline $\mathrm{Pt} / \mathrm{Al}_{2} \mathrm{O}_{3}$ aerogel & $0.37,0.10,0.26$ & $1.06,0.29,0.74$ \\
\hline $\mathrm{Pt} / \mathrm{SiO}_{2}{ }^{*}$ xerogel & 0.25 & 0.30 \\
\hline
\end{tabular}


1. Formation of nanoclusters by the inverse micelle technique.

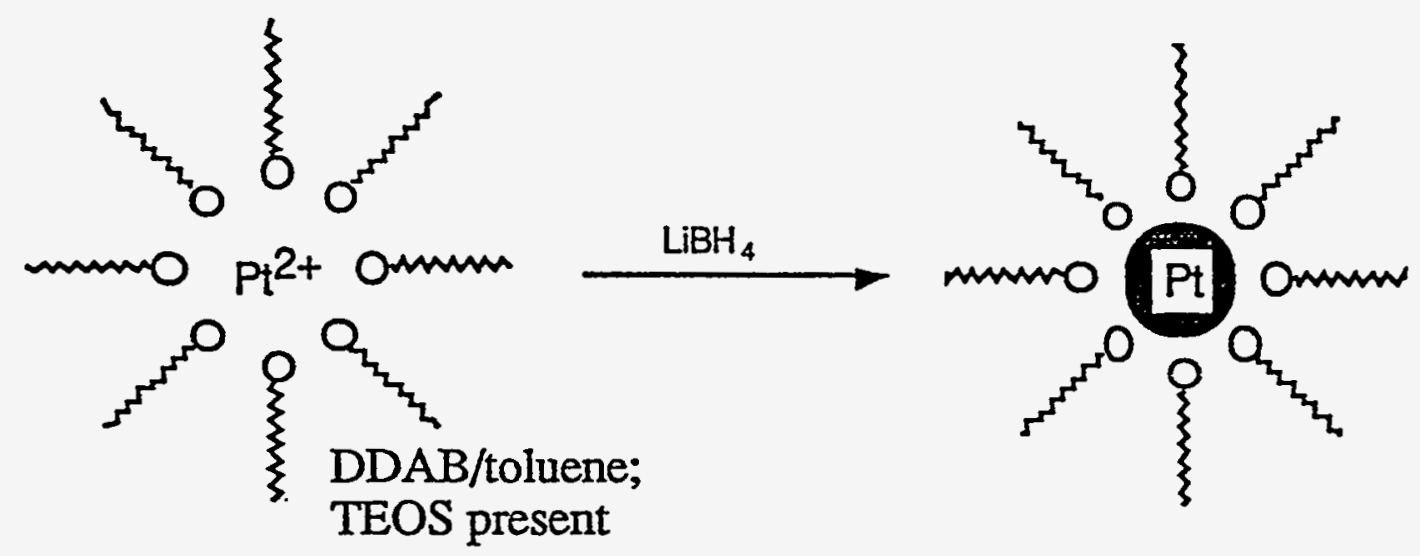

2. Formation of the gel around the nanoclusters.

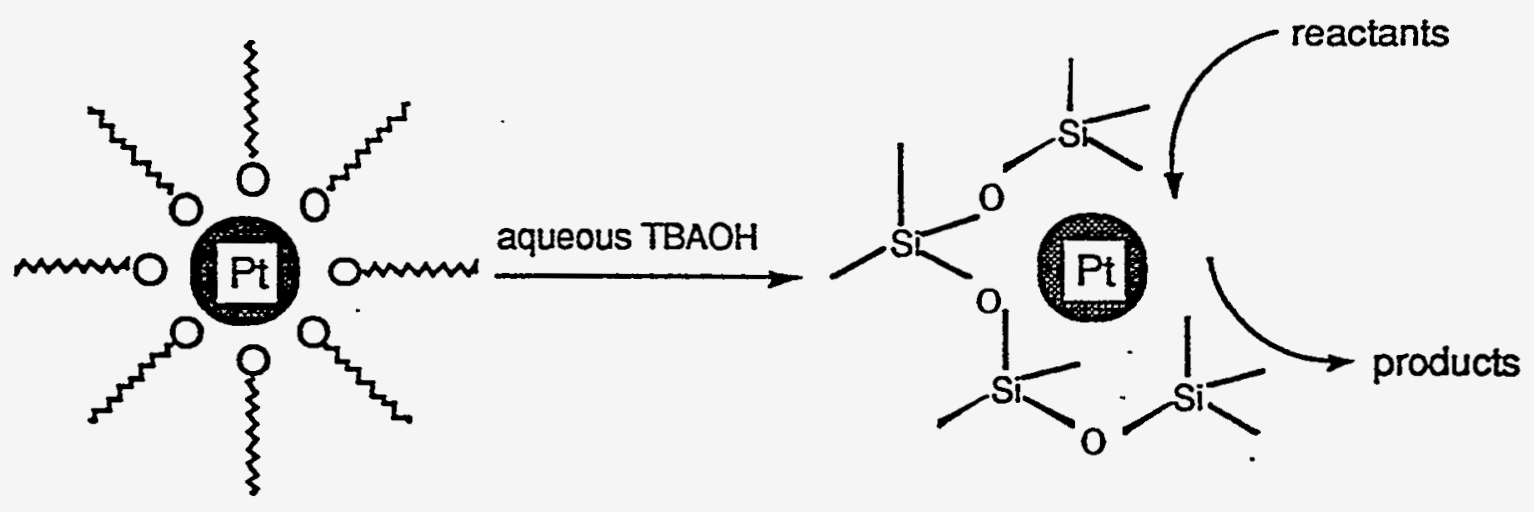

Figure 1. Schematic representation of the 'sequential' reaction method to form clusters embedded in gels. All reactants are initially present and the two reactions (metal salt reduction and sol-gel processing) are carried out in sequence. In the 'concurrent' reaction methods, the two reactions are carried out separately and the two products (clusters in inverse micelles and wet monoliths) are mixed. 

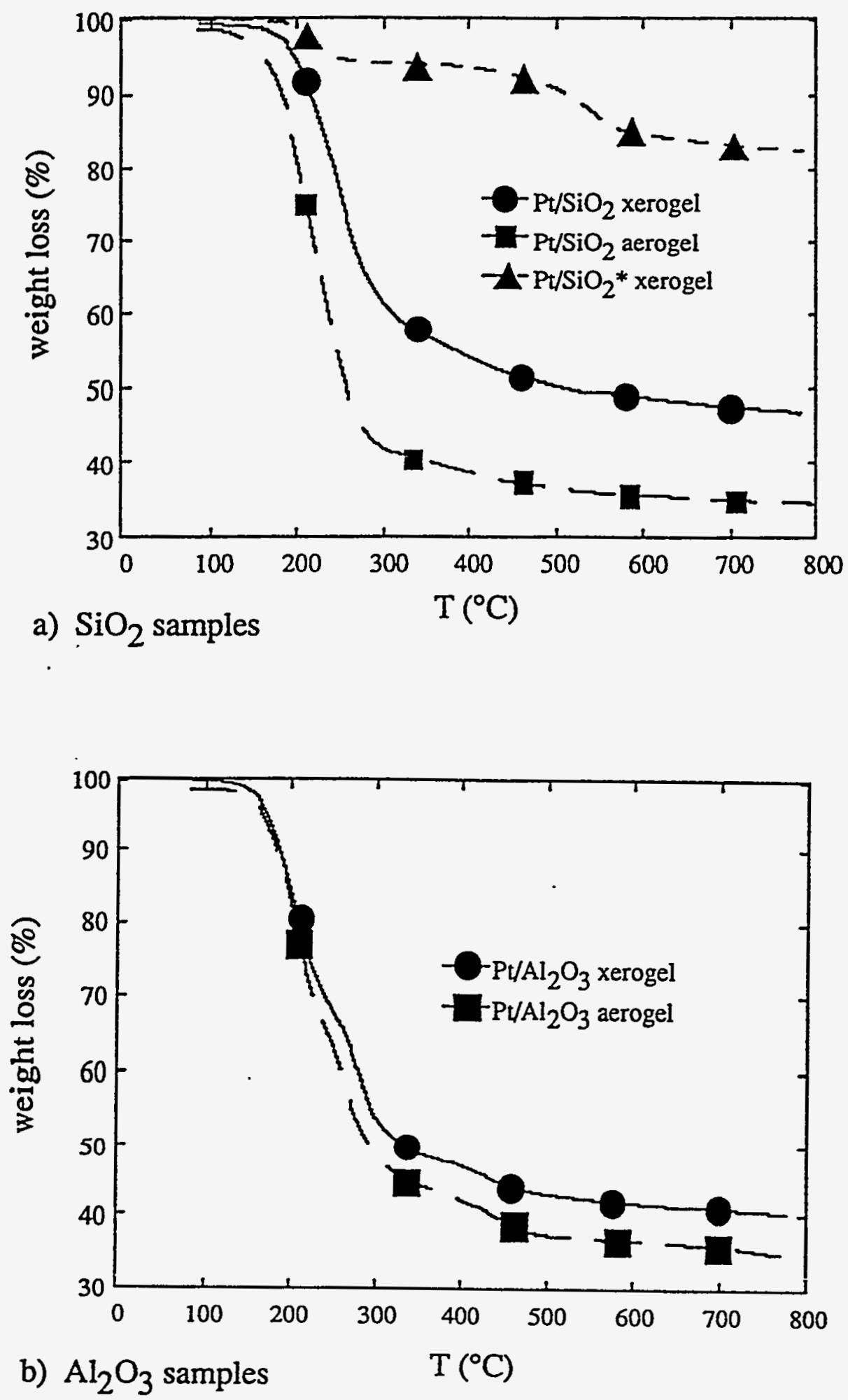

Figure 2. TGA results to determine weight loss with temperature. In sequentially produced samples, over $50 \mathrm{wt} . \%$ is lost due to unreacted gel precursor left by the inefficiency of solgel processing in inverse micelle solutions. The concurrently produced samples, using a well studied synthesis procedure to produce silica monoliths results in an efficient reaction and little loss. a) $\mathrm{SiO}_{2}$ samples. b) $\mathrm{Al}_{2} \mathrm{O}_{3}$ samples. 


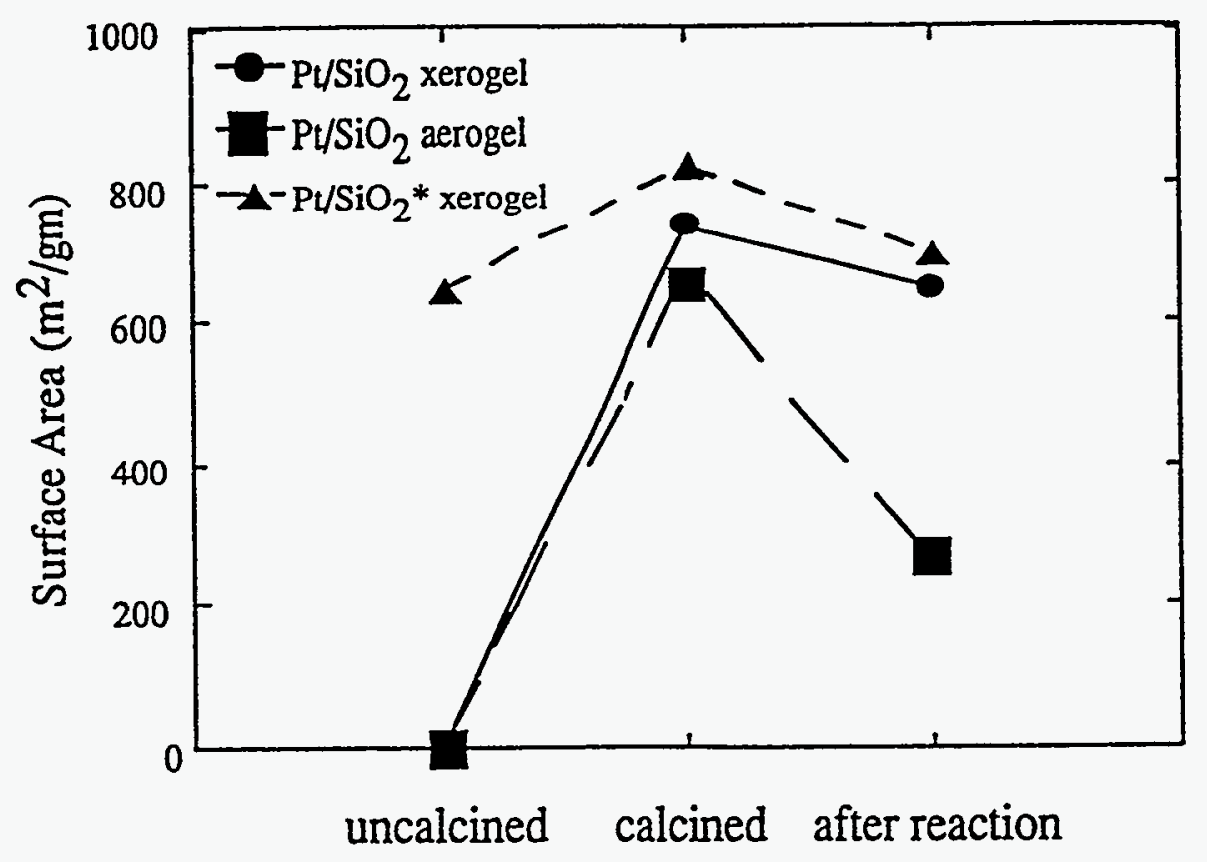

a) $\mathrm{SiO}_{2}$ samples

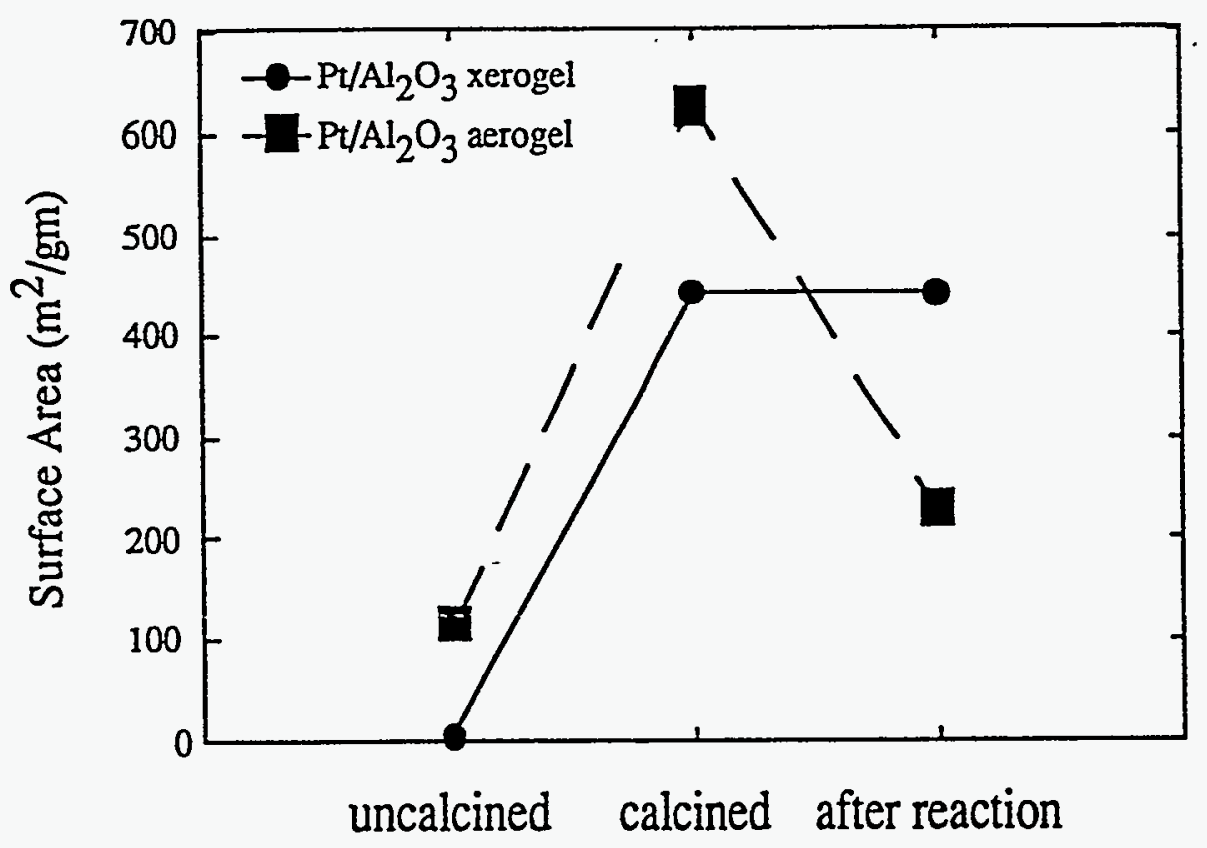

b) $\mathrm{Al}_{2} \mathrm{O}_{3}$ samples

Figure 3. $\mathrm{N}_{2}$ porosimetry results to determine surface area in dried, calcined, and reacted samples. In sequentially produced samples, removal of the unreacted TEOS upon calcination opens the pores and a large increase in surface area is observed. a) $\mathrm{SiO}_{2}$ samples. b) $\mathrm{Al}_{2} \mathrm{O}_{3}$ samples. 

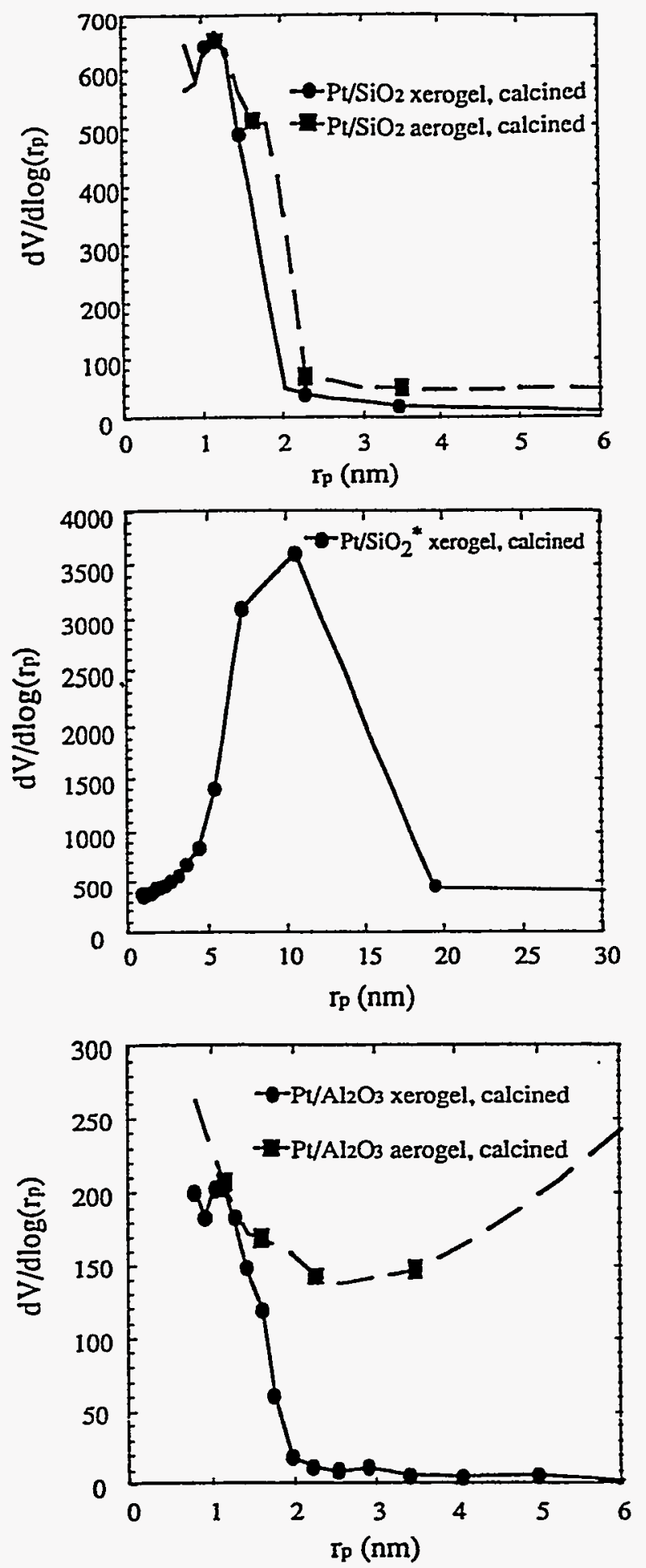

Figure 4. Pore size distributions calculated from the porosimetry results. In sequentially produced samples, the pores are small and monodisperse falling on the limit of what is defined as mesoporous (pore sizes approximately $1 \mathrm{~nm}$ in diameter). There is little effect of calcination. In the concurrently synthesized sample, pore sizes are larger and more disperse. The average pore size is approximately $6 \mathrm{~nm}$ in diameter before calcination (not shown) and grows upon calcination to approximately $11 \mathrm{~nm}$ in diameter. a) sequentially produced $\mathrm{SiO}_{2}$ samples. b) concurrently produced $\mathrm{SiO}_{2}$ sample. c) sequentially produced $\mathrm{Al}_{2} \mathrm{O}_{3}$ samples. 


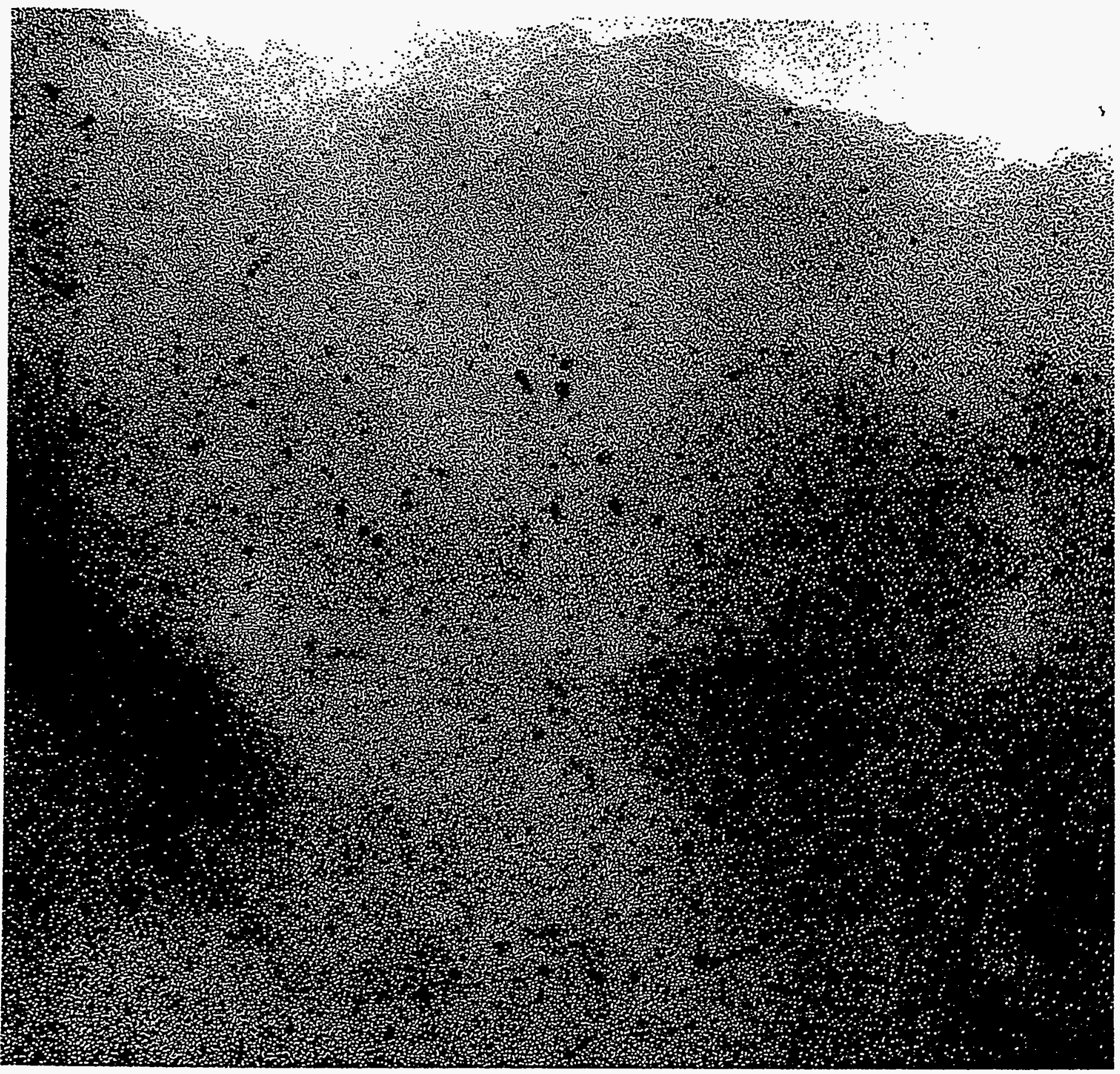

Pt / silica aerogel

Figure 5. Representative TEM picture of inverse micelle cluster, sol-gel derived materials. Sample shown is of $\mathrm{Pt}$ clusters on $\mathrm{SiO}_{2}$ aerogel support before calcination. 


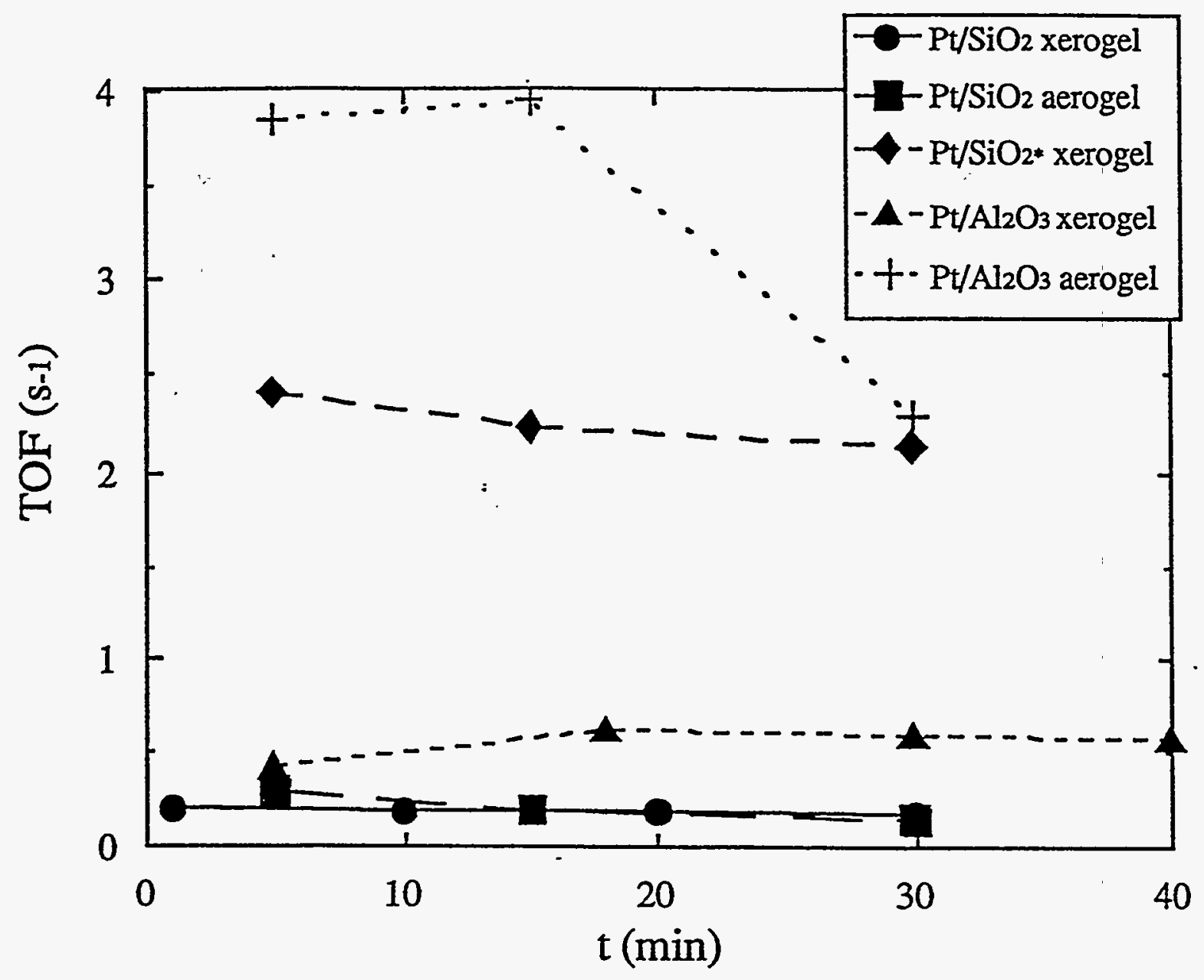

Figure 6. Activity of samples with respect to propane conversion to propene. Air regeneration and $\mathrm{H}_{2}$ reduction was used to achieve maximum activity for each sample. Two samples $\left(\mathrm{Pt}_{\mathrm{SiO}}{ }_{2}{ }^{\circ}\right.$ xerogel and $\mathrm{Pt} / \mathrm{Al}_{2} \mathrm{O}_{3}$ aerogel) show relatively large activities compared to previously published results. In addition, stability remains good up to 30 minutes (not tested longer). Previous results show complete loss of activity in less than 10 minutes unless a stabilizer is used. Increased stability could be due to prevention of char on the sol-gel derived supports. 


\title{
Chapter 6
}

\section{Novel Sol-Gel Based Nanocluster Materials as Catalysts in the Liquefaction of Coal}

\begin{abstract}
We continue our systematic characterization and catalyst activity testing of highly dispersed heterogeneous nanostructured materials by applying the above developed technology to the synthesis of iron based supported xerogel materials. Several iron based samples were synthesized and tested as catalysts in coal liquefaction.
\end{abstract}

\section{Results}

\section{Synthesis of Fe Based Nanostructured Heterogeneous Catalysts}

We have studied the synthesis of two different iron phases in the heterogeneous catalyst recipes. We have synthesized $\mathrm{Fe}^{0}$ particles and applied a new synthesis in an attempt to make $\mathrm{FeOOH}$. The synthesis of heterogeneous $\mathrm{Fe}^{0} /$ xerogel catalysts is fairly straightforward, because be originally synthesized $\mathrm{Fe}^{0}$ in inverse micelles by the reduction of $\mathrm{FeCl}_{3}$ with $\mathrm{LiBH}_{4}$. Some characterization is necessary, however, because of the addition of basic water in sol-gel processing. A series of four $\mathrm{Fe}^{0}$ samples of both silica and alumina have been synthesized in the past quarter.

The synthesis of $\mathrm{FeOOH}$ is desired, because $\mathrm{FeOOH}$ has been shown to be the best $\mathrm{Fe}$ based catalyst precursor for coal liquefaction. $\mathrm{FeOOH}$ in aqueous solutions is typically synthesized by regulating the $\mathrm{pH}$ to 8 and reacting an $\mathrm{Fe}$ salt with a solution of $\mathrm{H}_{2} \mathrm{O}_{2}$. The synthesis is tricky in inverse micelles with sol-gel processing, because $\mathrm{pH}$ in an apolar solvent is undefined and acids and bases catalysis sol-gel processing making $\mathrm{pH}$ regulation difficult. Nonetheless, we modified a literature preparation to our synthesis. We have not been able to identify FeOOH by xray diffraction. Modification to the synthesis is needed.

\section{Coal Liquefaction Testing}

We have completed initial coal liquefaction catalyst testing with the highly dispersed heterogeneous materials. The ultimate goal will be to develop plastic as a hydrogen source to increase the economic viability of coal liquefaction in a simple, single stage, single catalyst 
process. The highly dispersed heterogeneous catalysts are ideal for coal / plastic coprocessing, because they are bifunctional. The dispersed metal acts to hydrogenate the coal, and the silica or alumina support depolymerizes the plastic waste.

Results of the initial liquefaction tests are shown below. There are two trends that are important. First, overall conversion increases markedly with platinum. Strong effects on metal type indicate that despite being embedded in the support the metal is still highly dispersed with respect to the coal. Iron based materials are far less active than platinum. The second important result is that $\mathrm{Al}_{2} \mathrm{O}_{3}$ as a support consistently shows lower conversion to oil fractions than thermal runs. $\mathrm{Al}_{2} \mathrm{O}_{3}$ is a far better cracking catalyst than $\mathrm{SiO}_{2}$ due to surface acidity. We believe that cracking is occurring at a much faster rate than hydrogenation with $\mathrm{Al}_{2} \mathrm{O}_{3}$ causing regressive reactions with the coal. A support mixture of $\mathrm{SiO}_{2}$ and $\mathrm{Al}_{2} \mathrm{O}_{3}$ could be used to balance the hydrogenation and cracking rates to optimize conversion.

Metallic iron shows only an increase of roughly $7-10 \%$ in overall conversion compared to thermal. We are currently preparing $\mathrm{FeOOH}$ and $\mathrm{FeS}_{2}$ for testing in the heterogeneous recipes. It is stated in the results below that $\mathrm{FeOOH}$ was tested. A nominal recipe for $\mathrm{FeOOH}$ was attempted, but characterization does not indicate the presence of $\mathrm{FeOOH}$. The recipe must be adjusted further to the inverse micelle technique. $\mathrm{FeS}_{2}$ formation has been completed, but testing is not available yet.

Finally, two different $\mathrm{Fe} / \mathrm{Al}_{2} \mathrm{O}_{3}$ samples are shown in the results below. In the first as described in the experimental section, a two-step sequential reduction of an Fe salt followed by hydrolysis and condensation of aluminum alkoxides in a microemulsion forms nanoparticles in an alumina gel. In the second synthesis technique, nanoparticles and gels are formed in separate flasks, and the clusters are allowed to diffuse into the gel micropores upon mixing. Steric entrapment and thermal stability result from controlled drying and structural collapse of the gel. This latter methodology is currently being developed further.

The above results indicate two important trends (Figure 1). First, overall conversion increases markedly with platinum. Strong effects on metal type indicate that despite being embedded in the support the metal is still highly dispersed with respect to the coal. Iron based materials are far less active than platinum. The second important trend is that $\mathrm{Al}_{2} \mathrm{O}_{3}$ as a support consistently shows lower conversion to oil fractions (C7 sols) than thermal runs despite moderate increases in overall conversion. $\mathrm{Al}_{2} \mathrm{O}_{3}$ is a far better cracking catalyst than 
$\mathrm{SiO}_{2}$ due to surface acidity. We believe that cracking is occurring at a much faster rate than hydrogenation with $\mathrm{Al}_{2} \mathrm{O}_{3}$ causing regressive reactions with the coal.

It should be possible to optimize surface acidity to increase overall conversion and conversion to oil fractions by making supports of $\mathrm{SiO}_{2}$ and $\mathrm{Al}_{2} \mathrm{O}_{3}$ mixtures. In earlier work (Quarterly Report, 4/97), we described the synthesis of $\mathrm{SiO}_{2} / \mathrm{Al}_{2} \mathrm{O}_{3}$ support mixtures. This quarter we prepared metallic $\mathrm{Fe}$ clusters on a $10 \mathrm{wt} . \% \mathrm{Al}_{2} \mathrm{O}_{3} / \mathrm{SiO}_{2}$ support. The nanoparticles and gels were formed in separate flasks, and the clusters were allowed to diffuse into the gel micropores upon mixing. Steric entrapment and thermal stability resulted from controlled drying and structural collapse of the gel. This synthesis methodology provides the best catalyst as noticed from the differences between $\mathrm{Fe}^{0} / \mathrm{Al}_{2} \mathrm{O}_{3} 1$ and Fe $/ \mathrm{Al}_{2} \mathrm{O}_{3} 2$ (Figure 1. see Quarterly Report, 1/98 for details).

Coal liquefaction results indicate the effect of mixing $\mathrm{Al}_{2} \mathrm{O}_{3}$ and $\mathrm{SiO}_{2}$ in the support did not have the intended effect (Figure 2). Percentage of oil fractions is roughly the same as with pure $\mathrm{SiO}_{2}$ supports and slightly ahead of the thermal result. Overall conversion is down markedly, however. As shown in last quarters' results, overall conversion is highly dependent on metal type. Our leading explanation for this quarter's result is that the support acidity is effecting the nature of the metallic Fe composition, but clearly more investigation is needed. 


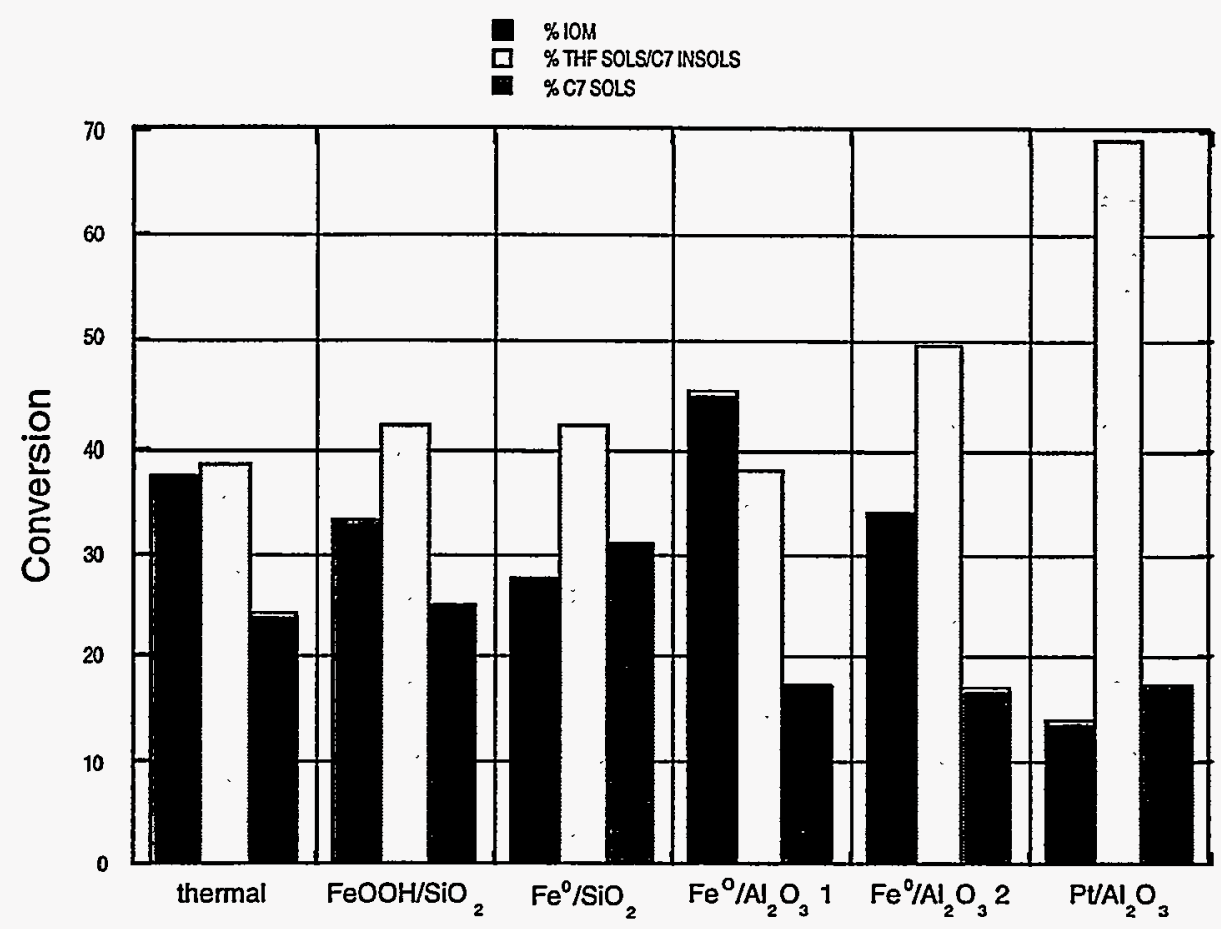

Figure 1. Conversion to THF sols and C7 sols in the liquefaction of DECS-17 coal. Highly dispersed Fe materials are compared to platinum, and the effects of $\mathrm{SiO}_{2}$ vs. $\mathrm{Al}_{2} \mathrm{O}_{3}$ support materials are determined. 


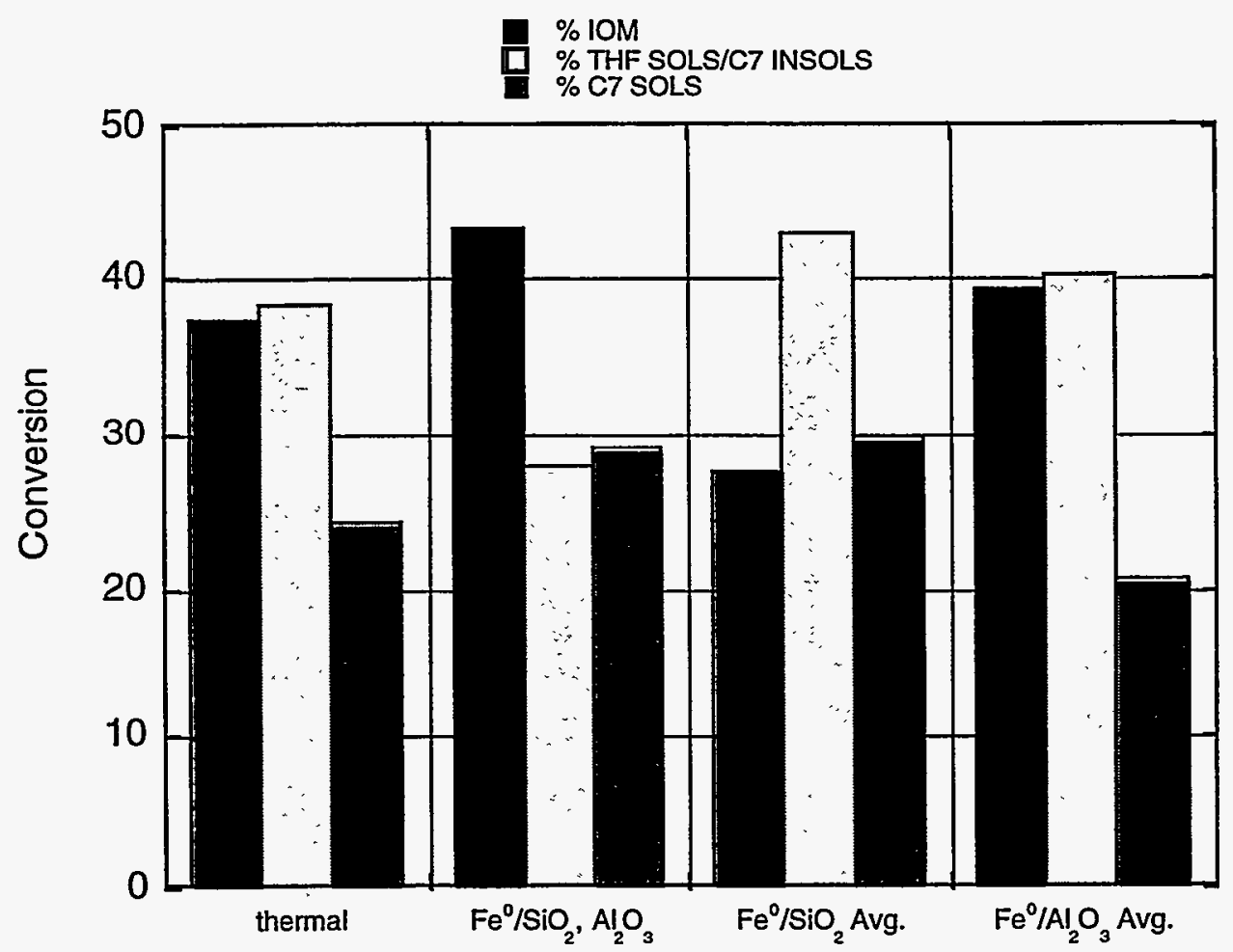




\section{Appendix}

\section{Publications}

1. Sault, A.G., Martino, A., Kawola, J.S., Boespflug, E., Novel Sol-Gel Based Nanocluster Catalysts in the Dehydrogenation of Propane, in preparation.

2. Martino, A., Sault, A.G., Kawola, J.S., Boespflug, E., Phillips, M.L.F., A Sintering Study of Novel Sol-Gel Based Nanocluster Catalysts, submitted to the Journal of Catalysis.

3. Martino, A., Yamanaka, S.A., Kawola, J.S., Showalter, S.K., Loy, D.A., Encapsulation of Metal Nanoclusters in Sol-Gel Materials via an Inverse Micelle Solution Synthesis, Technical Advance Disclosure, SD-5679,S-84,129, (1995).

4. Martino, A., Stoker, M., Hicks, M., Bartholomew, C.H., Sault, A.G., Kawola, J.S., "The Synthesis and Characterization of Iron Colloid Catalysts in Inverse Micelle Solutions", Applied Catalysis, in press.

5. Duan, Z., Hampden-Smith, M.J., Martino, A., Kawola, J.S., Preparation, Characterization, and Catalyst Testing of Highly Dispersed Rhodium Particles on Different Morphology Iron Oxide Supports, J. Mater. Chem., in preparation.

6. Martino, A., Yamanaka, S.A, Kawola, J.S., Loy, D.A., Encapsulation of Gold Nanoclusters in Silica Materials via an Inverse Micelle / Sol-Gel Synthesis, Chem. Mater., 9(2), 423, (1997).

7. Martino, A., Kawola, J.S., Yamanaka, S.A., Loy, D.A., Encapsulation of Metal Nanocluster Catalysts in Silica Materials via an Inverse Micelle / Sol-Gel Synthesis, Sandia Report, SAND97-1031*UC-1400, (1997).

8. Martino, A., Kawola, J.S., Andujar, L., Catalyst Testing of Highly-Dispersed Metal Nanoparticles for Coal Liquefaction and Coal/Waste Coprocessing. Coal Liquefaction \& Solid Fuels Contractors Review Conference, Pittsburgh, PA, (1997)

9. Martino, A.; Bartholomew, C.H.; Sault, A.G., Kawola, J.S.; Yamanaka, S.A., Synthesis and Characterization of Pseudo-Homogeneous and Heterogeneous Colloid Catalysts in Inverse Micelle Solutions, Proceedings Power and Fuel Systems Conference, (1996).

10. Martino, A., Stoker, M., Hicks, M., Bartholomew, C., Sault, A.G. Kawola, J.S., Synthesis and Characterization of Fe Colloid Catalysts in Inverse Micelles, Prepr. Pap.Am. Chem. Soc., Div. Petroleum Chem., 40(1), 78, (1995).

11. Martino, A., Stoker, M.; Hicks, M.; Bartholomew, C.H; Sault, A.G.; Kawola, J.S., Synthesis and Characterization of Fe Colloid Catalysts in Inverse Micelle Solutions, Proceedings Coal Liquefaction and Gas Conversion Conference, 715, (1995).

12. Wilcoxon, J.P., Bliss, D.E., Martin, J.E., Newcomer, P.P., Samara, G.A., Martino, A., Williamson, R.L., Photocatalytic Semiconductor Clusters for Fuel Production, Sandia Report, SAND95-2139*UC-404, (1995).

13. Martino, A., Wilcoxon, J.P., Kawola, J.S., Synthesis and Characterization of Coal Liquefaction Catalysts in Inverse Micelles, Energy \& Fuels, 8(6), 1289, (1994).

14. Wilcoxon, J.P., Martino, A., Klavetter, E., Sylwester, A.P., "Synthesis and Catalytic Properties of Metal and Semiconductor Nanoclusters", Ed. G. Hadjipanaysis, D. Siegel, Kluwer Academic Pub., pg. 770, (1994).

15. Martino, A., Wilcoxon, J.P., Sylwester, A.P., Kawola, J.S., Synthesis and Characterization of Iron and Iron Disulfide (Pyrite) Catalyst Particles in Inverse Micelles, Prepr. Pap.-Am. Chem. Soc. Div. Fuel Chem., 38(1), 357, (1993). 
16. Wilcoxon, J.P., Martino, A., Baughman, R.L., Klavetter, E., Sylwester, A.P., Synthesis of Transition Metal Clusters and Their Catalytic and Optical Properties, Mater. Res. Soc. Symp. Proc. (Nanophase and Nanocomposite Materials), 286, 131, (1993).

17. Yamanaka, S.A., Martino, A., Loy, D.A., Kawola, J.S., Non-Polar Processing of Silica Sol-Gel Materials via a Combined Inverse Micelle/Sol-Gel Technique. Materials Research Society Spring Meeting, San Francisco, CA, (1996).

18. Martino, A., Stoker, M., Hicks, M., Bartholomew, C.H., Sault, A.G., Kawola, J.S., The Synthesis and Characterization of Iron Colloid Catalysts in Inverse Micelle Solutions, American Institute of Chemical Engineers, Miami, FL, (1995).

19. Yamanaka, S.A., Martino, A., Loy, D.A., Kawola, J.S., Showalter, S.K., Encapsulated Metal Nanocluster Materials Prepared By A Novel Inverse Micelle/Sol-Gel Technique, Am. Chem. Soc. Meeting, Div. of Colloids, Chicago, IL, (1995).

20. Yamanaka, S.A., Martino, A., Loy D.A., Kawola, J.S., Showalter, S.K., Encapsulation of Metal Nanoclusters via a Combined Inverse Micelle/Sol-Gel Synthesis, 7th Annual Joint Meeting of the New Mexico Sections of the Materials Research Society and the American Ceramic Society, Albuquerque, NM, (1995).

21. Martino, A., Yamanaka, S.A., Kawola, J.S., Showalter, S.K., Loy, D.A., Encapsulation of Gold Clusters in Silica Materials via an Inverse Micelle/Sol-Gel Synthesis, Materials Research Society Fall Meeting, Boston, MA, (1995).

22. Yamanaka, S.A., Martino, A., Loy, D.A., Kawola, J.S., Showalter, S.K., Encapsulation of Metal Nanoclusters via a Combined Inverse Micelle/Sol-Gel Synthesis, 47th Pacific Coast Regional Meeting of the American Ceramic Society, Seattle, WA, (1995).

23. Martino, A., Stoker, M., Hicks, M., Bartholomew, C., Sault, A.G. Kawola, J.S., Synthesis and Characterization of Fe Colloid Catalysts in Inverse Micelles, North American Catalysis Society, Snowbird, UT, (1995).

24. Martino, A., Stoker, M., Hicks, M., Bartholomew, C., Sault, A.G. Kawola, J.S., Synthesis and Characterization of Fe Colloid Catalysts in Inverse Micelles, Am. Chem. Soc. Meeting, Div. Petroleum Chem., Anaheim, CA, (1995).

25. Martino, A., Wilcoxon, J.P., Sylwester, A.P., Kawola, J.S., Synthesis and Characterization of $\mathrm{Fe}$ and $\mathrm{FeS}_{2}$ (Pyrite) Catalyst Particles in Inverse Micelles, Am. Chem. Soc. Meeting, Div. Fuel Science, Denver, CO, (1993).

26. Wilcoxon, J.P., Martino, A., Klavetter, E., Sylwester, A.P., Synthesis and Catalytic Properties of Metal and Semiconductor Nanoclusters, Nanophase Materials, NATO ASI Meeting, Corfu, Greece, invited, (1993).

27. Wilcoxon, J.P., Martino, A., Nigrey, P.J., Sylwester, A.P., Inorganic and Organometallic Chemistry in Inverse Micelles: Formation and Characterization of Highly Dispersed Catalysts, Gordon Research Conference, Boston, MA, (1992).

28. Wilcoxon, J.P., Martino, A., Klavetter, E., Sylwester, A.P., Synthesis of Transition Metal Clusters and Their Catalytic and Optical Properties, Fall Materials Research Society Meeting, Boston, MA, (1992).

29. Martino, A., Wilcoxon, J.P., Formation and Characterization of Fe and $\mathrm{FeS}_{2}$ Catalyst Particles in Inverse Micelles, AIChE Annual Meeting, Miami Beach, FL, (1992).

30. Wilcoxon, J.P., Martino, A., Baughman R., Nigrey, P., Kawola, J., Klavetter, E., Salgado, L., Catalytic Behavior of Highly Dispersed Metal Clusters Synthesized in Inverse Micelles, Spring Materials Research Society Meeting, San Francisco, CA, (1992). 


\section{DISTRIBUTION:}

5 MS-0710 Anthony Martino

1 MS-0710 Jeffrey S. Kawola

$1 \quad$ MS-0710 Allen G. Sault

1 MS-0710 Alan P. Sylwester

1 MS-1349 Alan J. Hurd

1 MS-9018 Central Technical Files, 8940-2

2 MS-0899 Technical Library, 4916

1 MS-0619 Review and Approval Desk, 15102

For DOE/OSTI 